\title{
GEOMETRIC QUANTIZATION AND THE UNIVERSAL ENVELOPING ALGEBRA OF A NILPOTENT LIE GROUP
}

\author{
NIELS VIGAND PEDERSEN
}

\begin{abstract}
We study geometric quantization in connection with connected nilpotent Lie groups. First it is proved that the quantization map associated with a (real) polarized coadjoint orbit establishes an isomorphism between the space of polynomial quantizable functions and the space of polynomial quantized operators. Our methods allow noninductive proofs of certain basic facts from Kirillov theory. It is then shown how the quantization map connects with the universal enveloping algebra. This is the main result of the paper. Finally we show how one can explicitly compute global canonical coordinates on coadjoint orbits, and that this can be done simultaneously on all orbits contained in a given stratum of what we call "the fine $\mathscr{F}$-stratification of the dual of the Lie algebra". This is a generalization of a result of $M$. Vergne about simultaneous canonical coodinates for orbits in general position.
\end{abstract}

\section{INTRODUCTION}

Let $G$ be a connected Lie group with Lie algebra $\mathfrak{g}$, and let $O$ be the coadjoint orbit through the element $g \in \mathfrak{g}^{*}$. Further, let $\mathfrak{h}$ be a real polarization at $g$, and let $F$ be the corresponding $G$-invariant polarization of the symplectic manifold $O$. In this situation the space $\mathscr{E}_{F}^{1}(O)$ of smooth $F$-quantizable functions on $O$ is well defined (cf., e.g. [9]). Let $H_{0}$ be the analytic subgroup corresponding to $\mathfrak{h}$, and set $H=G_{g} H_{0}$, where $G_{g}$ is the stabilizer of $g$ in $G$, and suppose that there exists a unitary character $\chi: H \rightarrow \mathrm{T}$ such that $\chi(\exp X)=e^{i\langle g, X\rangle}$ for all $X \in \mathfrak{h}$. This means that the orbit $O$ is integral. We have in [9] defined the space $\mathscr{B}^{1}(G, \chi)$ of quantized operators. This space can be considered as the set of all differential operators of order at most one in the homogeneous line bundle with base $G / H$ defined by $\chi$. The main result of [9] was that the quantization map

$$
\delta_{\chi}: \mathscr{E}_{F}^{1}(O) \rightarrow \mathscr{B}^{1}(G, \chi)
$$

is a Lie algebra isomorphism.

Suppose now that $G$ is nilpotent and simply connected. Then the space of polynomial functions $\mathscr{P}(O)$ on $O$ is well defined, and therefore also the space $\mathscr{P}_{F}^{1}(O)\left(\subset \mathscr{E}_{F}^{1}(O)\right)$ of polynomial quantizable functions. Similarly we can speak

Received by the editors April 11, 1988.

1980 Mathematics Subject Classification (1985 Revision). Primary 22E27. 
of the space $\mathscr{A}^{1}(G, \chi)\left(\subset \mathscr{B}^{1}(G, \chi)\right)$ of polynomial quantized operators. In the first part of the paper we show that the image of the space of polynomial quantizable functions $\mathscr{P}_{F}^{1}(O)$ by the quantization map $\delta_{\chi}$ is equal to the space of polynomial quantized operators of order at most one $\mathscr{A}^{1}(G, \chi)$ (Theorem 2.4.1), i.e., $\delta_{\chi}$ defines a Lie algebra isomorphism

$$
\delta_{\chi}: \mathscr{P}_{F}^{1}(O) \rightarrow \mathscr{A}^{1}(G, \chi)
$$

Combined with Pukanszky's noninductive proof of the Kirillov character formula [10], our methods can be used to give noninductive proofs of two other basic facts from the Kirillov theory, namely that one can associate a well-defined class of irreducible representations of $G$ with each coadjoint orbit $O$ of $G$, and that the image of the universal enveloping algebra by the differential of such a representation can be identified with the algebra $\mathscr{A}\left(\mathbf{R}^{n}\right)$ of all polynomial differential operators on some space $\mathbf{R}^{n}$ (the Weyl algebra); in fact, we carry out this program in $\S 5$ (5.4.6).

Let now $\pi_{\chi}=$ ind $_{H \uparrow G} \chi$ be the induced representation of $\chi$. Then $\pi_{\chi}$ is a representative $r f$ the class of irreducible representations associated with $O$. Denoting by $d \pi_{\chi}$ une differential of $\pi_{\chi}$ considered as a representation of $U\left(g_{\mathbf{C}}\right)$, the universal enveloping algebra of the complexification $\mathfrak{g}_{\mathbf{C}}$ of $\mathfrak{g}$, we can for each $u \in U\left(\mathfrak{g}_{\mathbf{C}}\right)$ consider the operator $d \pi_{\chi}(u)$ as an element of the space $\mathscr{B}(G, \chi)$ corresponding to all differential operators in the line bundle mentioned above, and, in fact, it is easily seen that $d \pi_{\chi}(u)$ belongs to the space $\mathscr{A}(G, \chi)$ corresponding to all polynomial differential operators. Now from the theorem of Kirillov alluded to above, [5] (and its extension in [2]), we have that actually $d \pi_{\chi}\left(U\left(\mathfrak{g}_{\mathrm{C}}\right)\right)=\mathscr{A}(G, \chi)$. Let us now set $J_{F}^{1}(O)$ to be the set of elements in $U\left(\mathfrak{g}_{\mathrm{C}}\right)$ which are mapped by $d \pi_{\chi}$ to differential operators of order at most one, i.e., $J_{F}^{1}(O)=\left\{u \in U\left(\mathfrak{g}_{\mathrm{C}}\right) \mid d \pi_{\chi}(u) \in \mathscr{A}^{1}(G, \chi)\right\}$ (it is easily seen that the definition of $J_{F}^{1}(O)$ only depends on $\left.F\right)$. It then follows from Kirillov's theorem that $d \pi_{\chi}\left(J_{F}^{1}(O)\right)=\mathscr{A}^{1}(G, \chi)$. If we set $I(O)=\operatorname{ker}\left(d \pi_{\chi}\right)$ we can factor $d \pi_{\chi}$ through $I(O)$ to get an isomorphism

$$
d \tilde{\pi}_{\chi}: J_{F}^{1}(O) / I(O) \rightarrow \mathscr{A}^{1}(G, \chi)
$$

Now from the result above the quantization map

$$
\delta_{\chi}: \mathscr{P}_{F}^{1}(O) \rightarrow \mathscr{A}^{1}(G, \chi)
$$

is also an isomorphism. This means that there is a Lie algebra isomorphism $\tilde{\Phi}: \mathscr{P}_{F}^{1}(O) \rightarrow J_{F}^{1}(O) / I(O)$ which makes the following diagram commutative:

$$
\begin{array}{ccc}
\mathscr{P}_{F}^{1}(O) & \stackrel{\tilde{\Phi}}{\longrightarrow} & J_{F}^{1}(O) / I(O) \\
\delta_{\chi} \searrow & & \swarrow d \tilde{\pi}_{\chi} \\
& \mathscr{A}^{1}(G, \chi) &
\end{array}
$$

In $\S 3$ we consider the problem of finding an explicit description of $\tilde{\Phi}$. This problem has probably no noncomplicated solution for arbitrary polarizations. It 
turns out, however, that if $\mathfrak{h}$ is the Vergne polarization associated with a given Jordan-Hölder basis in $\mathfrak{g}$, then there is a simple explicit description of $\tilde{\Phi}$ in terms of the symmetrization map (Theorem 3.1.2). We use this to show how to construct a representation $\pi$ of $G$ on $L^{2}\left(\mathbf{R}^{d / 2}\right)$ associated with a given orbit $O$ for which not only $d \pi$ is very easily computable in geometric terms, but for which also a cross-section of the map $d \pi$ from $U\left(\mathfrak{g}_{\mathbf{C}}\right)$ to the algebra $\mathscr{A}\left(\mathbf{R}^{d / 2}\right)$ of all polynomial differential oparators on $\mathbf{R}^{d / 2}$ is very explicitly computable (Theorem 3.2.1). In $\S 6$ we give an example of such computations.

In several previous papers I have made essential use of a stratification, due to Pukanszky, of the dual of a nilpotent or solvable Lie algebra into finitely many strata. This stratification depends only on the choice of a Jordan-Hölder sequence $\mathscr{F}$ in $\mathfrak{g}$. We shall call it the coarse $\mathscr{F}$-stratification of $\mathfrak{g}^{*}$. For a long time I have known that a finer stratification can be useful, e.g., when considering characters of solvable Lie groups (cf. [8]). Here this finer stratification is defined in the nilpotent case $(\S 4)$. We shall call it the fine $\mathscr{F}$-stratification of $\mathfrak{g}^{*}$. Let $\mathscr{E}$ denote the index set of this finer stratification, and let $\Omega_{\varepsilon}$ denote the stratum corresponding to $\varepsilon \in \mathscr{E}$. We then have $\mathfrak{g}^{*}=\bigcup_{\varepsilon \in \mathscr{E}} \Omega_{\varepsilon}$. In $\S 5$ we show that there exists for each $\varepsilon \in \mathscr{E}$ rational functions $p_{1}^{\varepsilon}, \ldots, p_{d / 2}^{\varepsilon}, q_{1}^{\varepsilon}, \ldots, q_{d / 2}^{\varepsilon}$ on $\mathfrak{g}^{*}$ with the following properties: For each orbit $O$ contained in $\Omega_{\varepsilon}$ the restrictions $q_{1}^{O}, \ldots, q_{d / 2}^{O}$ of $q_{1}^{\varepsilon}, \ldots, q_{d / 2}^{\varepsilon}$ to $O$ and the restrictions $p_{1}^{O}, \ldots, p_{d / 2}^{O}$ of $p_{1}^{\varepsilon}, \ldots, p_{d / 2}^{\varepsilon}$ to $O$ define polynomial canonical coordinates on $O$ (Theorem 5.1.1). Actually we do more than that: We even give an algorithm for constructing these rational functions $p_{1}^{\varepsilon}, \ldots, p_{d / 2}^{\varepsilon}, q_{1}^{\varepsilon}, \ldots, q_{d / 2}^{\varepsilon}$. This means that we have in particular an algorithm for explicitly constructing global canonical coordinates on each coadjoint orbit of $G$. In $\S 6$ we give an example of calculations carried out by means of this algorithm.

In [11] M. Vergne proved that if $G$ is nilpotent, then there exists a Zariski open, $G$-invariant subset $\Omega$ of $\mathfrak{g}^{*}$ and rational functions $\tilde{p}_{1}, \ldots, \tilde{p}_{d / 2}, \tilde{q}_{1}, \ldots$, $\tilde{q}_{d / 2}$ such that these functions when restricted to any coadjoint orbit $O$ contained in $\Omega$ define canonical coordinates on $O$. This result is contained as a special case (corresponding to generic orbits) in our Theorem 5.1.1. Our method of proof is entirely different from the one in [11]; in particular we proceed constructively, without induction on the dimension of the group.

\section{Preliminaries}

In the following we shall discuss various questions related to the fact that a connected, simply connected nilpotent Lie group can be considered as $\mathbf{R}^{m}$ equipped with a group product which can be expressed by polynomial functions. The main result is Proposition 1.7.4. For the convenience of the reader we repeat certain well-known facts for which there exist only scattered references.

1.1. Polynomial structures. In dealing with nilpotent Lie groups we shall find the following terminology useful. 
Let $M$ be an $m$-dimensional smooth manifold with atlas $\mathscr{A}_{M}$. We say that a subatlas $\mathscr{A}_{M}^{0}$ of $\mathscr{A}_{M}$ defines a polynomial structure on $M$ if (i) all charts in $\mathscr{A}_{M}^{0}$ are defined on all of $M$ and have image $\mathbf{R}^{m}$, and (ii) for any two charts $\sigma, \tau$ in $\mathscr{A}_{M}^{0}$ we have that $\sigma \circ \tau^{-1}: \mathbf{R}^{m} \rightarrow \mathbf{R}^{m}$ and $\tau \circ \sigma^{-1}: \mathbf{R}^{m} \rightarrow \mathbf{R}^{m}$ are both polynomial maps. So, if $M$ carries a polynomial structure it is in particular diffeomorphic to $\mathbf{R}^{m}$.

If $M$ and $N$ are smooth manifolds of dimension $m$ and $n$, respectively, equipped with polynomial structures $\mathscr{A}_{M}^{0}$ and $\mathscr{A}_{N}^{0}$, respectively, we say that a map $f: M \rightarrow N$ is polynomial if the map $\tau \circ f \circ \sigma^{-1}: \mathbf{R}^{m} \rightarrow \mathbf{R}^{n}$ is a polynomial map for one (hence all) chart(s) $\sigma \in \mathscr{A}_{M}^{0}$ and one (hence all) chart(s) $\tau \in \mathscr{A}_{N}^{0}$. We say that $f$ is a bi-polynomial diffeomorphism if $f$ is a diffeomorphism and if both $f$ and $f^{-1}$ are polynomial.

If $M$ and $N$ are smooth manifolds with polynomial structures, then the smooth manifold $M \times N$ carries a natural polynomial structure.

The Euclidian space $\mathbf{R}^{m}$ carries a natural polynomial structure. A bi-polynomial diffeomorphism $f: \mathbf{R}^{m} \rightarrow \mathbf{R}^{m}$ is a diffeomorphism such that both $f$ and $f^{-1}$ are polynomial maps. A finite dimensional real vector space $V$ over $\mathbf{R}$ likewise carries a natural polynomial structure.

Let $M$ be a smooth manifold equipped with a polynomial structure $\mathscr{A}_{M}^{0}$. A chart $\sigma$ on $M$ is said to be a polynomial chart, if $\sigma$ is a bi-polynomial diffeomorphism from $M$ onto $\mathbf{R}^{m}$. The space of all polynomial functions $f: M \rightarrow \mathbf{C}$ is denoted $\mathscr{P}(M)$. Any object defined on $\mathbf{R}^{m}$ which is invariant under bi-polynomial diffeomorphisms can also be defined on $M$. In particular we can speak of rapidly decreasing functions $\mathscr{S}(M)$ on $M$, tempered distributions $\mathscr{S}^{\prime}(M)$, smooth functions of polynomial growth $\mathscr{O}_{M}(M)$, polynomial differential operators $\mathscr{A}(M)$ (of order at most $k, \mathscr{A}^{k}(M)$ ), polynomial vector fields $\mathscr{U}(M)$, polynomial differential forms etc.

1.2. The polynomial structure on a connected, simply connected nilpotent Lie group. Let $G$ be a connected, simply connected nilpotent Lie group with Lie algebra $\mathfrak{g}$. It is well known that the exponential map exp: $\mathfrak{g} \rightarrow G$ is a diffeomorphism. We shall equip $G$ with the polynomial structure obtained by transporting the natural polynomial structure on $\mathfrak{g}$ to $G$ via the exponential map.

Let $\mathscr{B}: X_{1}, \ldots, X_{m}$ be a basis in $\mathfrak{g}$. We shall say that the basis $\mathscr{B}$ is of subalgebra type if the subspace $\mathfrak{g}_{j}=\mathbf{R} X_{1} \oplus \cdots \oplus \mathbf{R} X_{j}$ is a subalgebra in $\mathfrak{g}$ for each $j=1, \ldots, m$. If $\mathscr{B}$ is such a basis then actually $\mathfrak{g}_{j-1}$ is an ideal in $\mathfrak{g}_{j}$ for each $j=2, \ldots, m$. Recall that the basis $\mathscr{B}$ is said to be a Jordan-Hölder basis if $\mathfrak{g}_{j}$ is an ideal in $\mathfrak{g}$ for each $j=1, \ldots, m$. 
Let $\mathscr{B}: X_{1}, \ldots, X_{m}$ be a basis in $\mathfrak{g}$ of subalgebra type. It is well-known that the map $\mathbf{R}^{m} \rightarrow G:\left(x_{1}, \ldots, x_{m}\right) \rightarrow \exp x_{m} X_{m} \cdots \exp x_{1} X_{1}$ is a diffeomorphism. We define $\sigma_{G}^{\mathscr{B}}$ to be the inverse of this map, i.e.,

$$
\sigma_{G}^{\mathscr{B}}: \exp x_{m} X_{m} \cdots \exp x_{1} X_{1} \rightarrow\left(x_{1}, \ldots, x_{m}\right),
$$

and $\sigma_{G}^{\mathscr{B}}$ is then a chart on $G$.

Lemma 1.2.1. Write

$$
\begin{aligned}
& \left(\sigma_{G}^{\mathscr{B}}\right)^{-1}\left(x_{1}, \ldots, x_{m}\right)=\exp \left(Q_{1}(x) X_{1}+\cdots+Q_{m}(x) X_{m}\right), \\
& \sigma_{G}^{\mathscr{B}}\left(\exp \left(u_{1} X_{1}+\cdots+u_{m} X_{m}\right)\right)=\left(P_{1}(u), \ldots, P_{m}(u)\right),
\end{aligned}
$$

$x=\left(x_{1}, \ldots, x_{m}\right) \in \mathbf{R}^{m}, u=\left(u_{1}, \ldots, u_{m}\right) \in \mathbf{R}^{m}$. If $\mathscr{B}$ is a Jordan-Hölder basis then $Q_{1}, \ldots, Q_{m}, P_{1}, \ldots, P_{m}$ are polynomial functions and we have

$$
\begin{gathered}
Q_{j}(x)=x_{j}+\bar{Q}_{j}\left(x_{j+1}, \ldots, x_{m}\right), \\
P_{j}(u)=u_{j}+\bar{P}_{j}\left(u_{j+1}, \ldots, u_{m}\right)
\end{gathered}
$$

for each $j=1, \ldots, m$, where $\bar{Q}_{j}, \bar{P}_{j}$ are polynomial functions with properties as indicated (i.e., depending only on the variables $x_{j+1}, \ldots, x_{m}, u_{j+1}, \ldots, u_{m}$, respectively).

Proof. The statement about $Q_{1}, \ldots, Q_{m}$ follows immediately from the Campbell-Baker-Hausdorff formula, and the statement about $P_{1}, \ldots, P_{m}$ is then clear, cf. [6].

Corollary 1.2.2. If $\mathscr{B}$ is a Jordan-Hölder basis then $\sigma_{G}^{\mathscr{B}}$ is a polynomial chart.

For $x, y \in \mathbf{R}^{m}$ we set $x \cdot y=\sigma_{G}^{\mathscr{B}}\left(\left(\sigma_{G}^{\mathscr{B}}\right)^{-1}(x)\left(\sigma_{G}^{\mathscr{B}}\right)^{-1}(y)\right)$. In other words, we identify $G$ with $\mathbf{R}^{m}$ via the chart $\sigma_{G}^{\mathscr{B}}$, and carry the group product on $G$ over to $\mathbf{R}^{m}$. With this notation we have

Lemma 1.2.3. If $\mathscr{B}$ is a Jordan-Hölder basis then the function $P(x, y)=x \cdot y$ is a polynomial function on $\mathbf{R}^{m} \times \mathbf{R}^{m}$, and writing

$$
P(x, y)=\left(P_{1}(x, y), \ldots, P_{m}(x, y)\right)
$$

we have

$$
\begin{aligned}
P_{j}(x, y) & =P_{j}\left(x_{j}, \ldots, x_{m}, y_{j}, \ldots, y_{m}\right) \\
& =x_{j}+y_{j}+\bar{P}_{j}\left(x_{j+1}, \ldots, x_{m}, y_{j+1}, \ldots, y_{m}\right),
\end{aligned}
$$

for $1 \leq j \leq m$, where we have written $x=\left(x_{1}, \ldots, x_{m}\right), y=\left(y_{1}, \ldots, y_{m}\right)$, and where $\bar{P}_{j}$ are polynomial functions with properties as indicated.

Proof. This follows immediately from the Campbell-Baker-Hausdorff formula and Lemma 1.2.1, cf. [6].

The following result will be useful later. Let $e$ be a nonempty subset of $\{1, \ldots, m\}$, and set $e^{\prime}$ to be the complement of $e$ in $\{1, \ldots, m\}$. 
Lemma 1.2.4. There exist unique functions $S: \mathbf{R}^{m} \rightarrow \mathbf{R}^{m}$ and $K: \mathbf{R}^{m} \rightarrow \mathbf{R}^{m}$ such that

$$
\left\{\begin{array}{c}
S_{j}=0 \quad \text { for } j \in e^{\prime}, \\
K_{j}=0 \quad \text { for } j \in e, \\
x=S(x) \cdot K(x) .
\end{array}\right.
$$

These functions are polynomial functions, and

$$
\begin{aligned}
& S_{j}(x)=x_{j}+\bar{S}_{j}\left(x_{j+1}, \ldots, x_{m}\right) \quad \text { for } j \in e, \\
& K_{j}(x)=x_{j}+\bar{K}_{j}\left(x_{j+1}, \ldots, x_{m}\right) \quad \text { for } j \in e^{\prime},
\end{aligned}
$$

where $\bar{S}_{j}$ and $\bar{K}_{j}$ are polynomial functions with properties as indicated.

Proof. Suppose first that we have found functions satisfying $(*)$. We shall prove by induction on $j$ (starting at $m$ and ending at 1 ) that $S_{j}, K_{j}, j=m, \ldots, 1$, are uniquely determined and that they are polynomial functions with properties as indicated. We have for each $j=m, \ldots, 1$

$$
\begin{aligned}
x_{j} & =P_{j}(S(x), K(x)) \\
& =S_{j}(x)+K_{j}(x)+\bar{P}_{j}\left(S_{j+1}(x), \ldots, S_{m}(x), K_{j+1}(x), \ldots, K_{m}(x)\right) .
\end{aligned}
$$

In particular $x_{m}=S_{m}(x)+K_{m}(x)$. Therefore, if $m \in e$ we have $S_{m}(x)=x_{m}$ and $K_{m}(x)=0$; if $m \in e^{i}$ we have $S_{m}(x)=0$ and $K_{m}(x)=x_{m}$. This means that we can start the induction (at $m$ and descending to 1). Suppose now that the claim has been proved for all values greater than $j$. We shall then show that it is valid also for the value $j$. If $j \in e$ we have $K_{j}(x)=0$ and by the induction hypothesis

$$
\begin{aligned}
S_{j}(x)=x_{j}-\bar{P}_{j}\left(S_{j+1}\left(x_{j+1}, \ldots, x_{m}\right), \ldots, S_{m}\left(x_{m}\right)\right. \\
\left.K_{j+1}\left(x_{j+1}, \ldots, x_{m}\right), \ldots, K_{m}\left(x_{m}\right)\right)
\end{aligned}
$$

which shows that $S_{j}$ is uniquely determined and that it is a polynomial function, since $S_{m}, \ldots, S_{j+1}, K_{m}, \ldots, K_{j+1}$ are uniquely determined and polynomial functions, and that $S_{j}(x)=x_{j}+\bar{S}\left(x_{j+1}, \ldots, x_{m}\right)$. Similarly for $K_{j}$ if $j \in e^{\prime}$.

The existence of the functions $S$ and $K$ are proved similarly by recursion, starting with $S_{m}, K_{m}$ and descending to $S_{1}, K_{1}$.

Suppose that $\mathscr{B}^{\prime}: X_{1}^{\prime}, \ldots, X_{m}^{\prime}$ is another basis in $\mathfrak{g}$ and suppose that $X_{j}^{\prime}-$ $X_{j} \in \mathfrak{g}_{j-1}$ for $j=1, \ldots, m$.

Lemma 1.2.5. Set $T=\left(T_{1}, \ldots, T_{m}\right): \mathbf{R}^{m} \rightarrow \mathbf{R}^{m}$ to be the polynomial function $\sigma_{G}^{\mathscr{B}^{\prime}} \circ \sigma_{G}^{\mathscr{B}}{ }^{-1}$. If $\mathfrak{g}_{0}, \ldots, \mathfrak{g}_{m}$ is a Jordan-Hölder sequence we have

$$
T_{j}(x)=x_{j}+\bar{T}_{j}\left(x_{j+1}, \ldots, x_{m}\right)
$$


$x=\left(x_{1}, \ldots, x_{m}\right)$, where $\bar{T}_{j}$ are polynomial functions with properties as indicated.

Proof. This follows immediately from Lemma 1.2.1 and Lemma 1.2.3.

For $x \in \mathbf{R}^{m}$ we set $x^{-1}=\sigma_{G}^{\mathscr{B}}\left(\left(\left(\sigma_{G}^{\mathscr{B}}\right)^{-1}(x)\right)^{-1}\right)$. With this notation we have Lemma 1.2.6. If $\mathscr{B}$ is a Jordan-Hölder basis then the function $I(x)=x^{-1}$ is a polynomial function on $\mathbf{R}^{m}$, and writing $I(x)=\left(I_{1}(x), \ldots, I_{m}(x)\right)$ we have

$$
I_{j}(x)=-x_{j}+\bar{I}_{j}\left(x_{j+1}, \ldots, x_{m}\right),
$$

where we have written $x=\left(x_{1}, \ldots, x_{m}\right)$, and where $\bar{I}_{j}$ are polynomial functions with properties as indicated.

Proof. This follows immediately from Lemma 1.2.3.

Corollary 1.2.7. The group operations $G \times G \rightarrow G: \quad(s, t) \rightarrow$ st and $G \rightarrow G$ : $s \rightarrow s^{-1}$ are polynomial maps.

Suppose that $\mathfrak{g}_{0}$ is a subalgebra in $\mathfrak{g}$ of codimension 1 , and let $X \in \mathfrak{g} \backslash \mathfrak{g}_{0}$. Denoting by $G_{0}$ the analytic subgroup corresponding to $\mathfrak{g}_{0}$ it is well known that the map $\mathbf{R} \times G_{0} \rightarrow G$ given by $(t, s) \rightarrow \exp t X s$ is a diffeomorphism. Actually we have

Lemma 1.2.8. The map

$$
\mathbf{R} \times G_{0} \rightarrow G:(t, s) \rightarrow \exp t X s
$$

is a bi-polynomial diffeomorphism.

Proof. Let $\sigma$ be some polynomial chart on $G$ and let $\tau$ be some polynomial chart on $G_{0}$. We have to show that the map $\varphi: \mathbf{R}^{m} \rightarrow \mathbf{R}^{m}$ given by $\varphi:\left(t, x_{1}, \ldots, x_{m-1}\right) \rightarrow \sigma\left(\exp t X \tau^{-1}\left(x_{1}, \ldots, x_{m-1}\right)\right.$ is a bi-polynomial diffeomorphism. Now since we know that actually $\mathfrak{g}_{0}$ is an ideal in $\mathfrak{g}$ we can find a Jordan-Hölder basis $\mathscr{B}: X_{1}, \ldots, X_{m}$ in $\mathfrak{g}$ such that $\mathscr{B}_{0}: X_{1}, \ldots, X_{m-1}$ is a Jordan-Hölder basis in $\mathfrak{g}_{0}$ and such that $X_{m}=X$. Using $\sigma=\sigma_{G}^{\mathscr{B}}$ and $\tau=\sigma_{G_{0}}^{\mathscr{B}_{0}}$ we get that $\varphi\left(t, x_{1}, \ldots, x_{m-1}\right)=\left(x_{1}, \ldots, x_{m-1}, t\right)$, so $\varphi$ is polynomial. This ends the proof of the lemma.

Lemma 1.2.9. The chart $\sigma_{G}^{\mathscr{B}}$ is a polynomial chart for any basis $\mathscr{B}$ of subalgebra type.

Proof. The proof is by induction on the dimension of $G$. The case $\operatorname{dim} G=1$ being trivial, assume the result has been proved for all dimensions less that $m=\operatorname{dim} G$. Let $\mathscr{B}: X_{1}, \ldots, X_{m}$ be the given basis of subalgebra type, and set $\mathfrak{g}_{0}=\mathbf{R} X_{1} \oplus \cdots \oplus \mathbf{R} X_{m-1}$. Then $\mathfrak{g}_{0}$ is a subalgebra of codimension 1 in $\mathfrak{g}$. Moreover, $\mathscr{B}_{0}: X_{1}, \ldots, X_{m-1}$ is a basis in $\mathfrak{g}_{0}$ of subalgebra type, and $X_{m} \notin \mathfrak{g}_{0}$. It follows from the induction hypothesis that $\sigma_{G_{0}}^{\mathscr{B}_{0}}$ is a polynomial chart on $G_{0}=\exp \mathfrak{g}_{0}$, and since

$$
\left(\sigma_{G}^{\mathscr{B}}\right)^{-1}\left(x_{1}, \ldots, x_{m}\right)=\exp x_{m} X_{m}\left(\sigma_{G_{0}}^{\mathscr{B}_{0}}\right)^{-1}\left(x_{1}, \ldots, x_{m-1}\right)
$$

it follows from Lemma 1.2.8 that $\sigma_{G}^{\mathscr{B}}$ is a polynomial chart. 
1.3. The polynomial structure on a simply connected homogeneous space of a nilpotent Lie group. Let $G$ be a connected, simply connected nilpotent Lie group with Lie algebra $\mathfrak{g}$, let $\mathfrak{h}$ be a subalgebra of $\mathfrak{g}$, and let $H$ be the analytic subgroup corresponding to $\mathfrak{h}$. We shall equip $G / H$ with a polynomial structure.

First some notation. Let $Y_{1}, \ldots, Y_{n}$ be elements in $\mathfrak{g}$. We say that $Y_{1}, \ldots$, $Y_{n}$ is a basis in $\mathfrak{g}(\bmod \mathfrak{h})$ if $Y_{1}, \ldots, Y_{n}$ is a linearly independent set and if $\mathfrak{g}=\mathbf{R} Y_{1} \oplus \cdots \oplus \mathbf{R} Y_{n} \oplus \mathfrak{h}$. We say that $Y_{1}, \ldots, Y_{n}$ is a basis in $\mathfrak{g}(\bmod \mathfrak{h})$ of subalgebra type if each of the subspaces $\mathfrak{h}_{j}=\mathbf{R} Y_{1} \oplus \cdots \oplus \mathbf{R} Y_{j} \oplus \mathfrak{h}$ is a subalgebra in $\mathfrak{g}$. If this is so, $\mathfrak{h}_{j-1}$ is actually an ideal in $\mathfrak{h}_{j}, j=0,1, \ldots, n$ (where $\left.\mathfrak{h}_{0}=\mathfrak{h}\right)$.

Let $Y_{1}, \ldots, Y_{n}$ be a basis in $\mathfrak{g}(\bmod \mathfrak{h})$ of subalgebra type. Then it is well-known that the map $\sigma: G / H \rightarrow \mathbf{R}^{n}$ given by

$$
\sigma: \exp t_{n} Y_{n} \cdots \exp t_{1} Y_{1} H \rightarrow\left(t_{1}, \ldots, t_{n}\right)
$$

is a diffeomorphism. Suppose now that $Z_{1}, \ldots, Z_{n}$ is another basis in $\mathfrak{g}$ $(\bmod \mathfrak{h})$ of subalgebra type, and define analogously the map $\tau: G / H \rightarrow \mathbf{R}^{n}$ by

Then we have

$$
\tau: \exp t_{n} Z_{n} \cdots \exp t_{1} Z_{1} H \rightarrow\left(t_{1}, \ldots, t_{n}\right)
$$

Lemma 1.3.1. The maps $\sigma \circ \tau^{-1}: \mathbf{R}^{n} \rightarrow \mathbf{R}^{n}$ and $\tau \circ \sigma^{-1}: \mathbf{R}^{n} \rightarrow \mathbf{R}^{n}$ are polynomial.

Proof. Let $W_{1}, \ldots, W_{m-n}$ be a basis in $\mathfrak{h}$ of subalgebra type. Then $W_{1}, \ldots$, $W_{m-n}, Y_{1}, \ldots, Y_{n}$ and $W_{1}, \ldots, W_{m-n}, Z_{1}, \ldots, Z_{n}$ are both bases of subalgebra type in $\mathfrak{g}$, and the result now follows from Lemma 1.2.9.

Lemma 1.3.1 allows us to define a polynomial structure on $G / H$ consisting of all possible charts $\sigma$ arising as above.

Lemma 1.3.2. A function $\varphi: G / H \rightarrow \mathrm{C}$ is a polynomial function if and only if the function $\tilde{\varphi}: G \rightarrow \mathbf{C}$ given by $\tilde{\varphi}(s)=\varphi(s H)$ is a polynomial function on $G$. Proof. Let $Y_{1}, \ldots, Y_{n}$ be a basis in $\mathfrak{h}$ of subalgebra type, and let $Y_{n+1}, \ldots, Y_{m}$ be a basis in $\mathfrak{g}(\bmod \mathfrak{h})$ of subalgebra type. Then $Y_{1}, \ldots, Y_{m}$ is a basis in $\mathfrak{g}$ of subalgebra type. Let $\tilde{\sigma}$ be the chart on $G / H$ associated with the basis $Y_{1}, \ldots, Y_{m}$, and let $\sigma$ be the chart on $G / H$ associated with the basis $Y_{n+1}, \ldots, Y_{m}(\bmod \mathfrak{h})($ see $\S 1.3)$. We then have

$$
\begin{aligned}
\tilde{\varphi} \circ \tilde{\sigma}^{-1}\left(t_{1}, \ldots, t_{m}\right) & =\tilde{\varphi}\left(\exp t_{m} Y_{m} \cdots \exp t_{1} Y_{1}\right) \\
& =\varphi\left(\exp t_{m} Y_{m} \cdots \exp t_{1} Y_{1} H\right) \\
& =\varphi\left(\exp t_{m} Y_{m} \cdots \exp t_{n+1} Y_{n+1} H\right) \\
& =\varphi \circ \sigma^{-1}\left(t_{n+1}, \ldots, t_{m}\right) .
\end{aligned}
$$

This shows that $\tilde{\varphi} \circ \tilde{\sigma}^{-1}$ is a polynomial function if and only if $\varphi \circ \sigma^{-1}$ is a polynomial function, and this ends the proof of the lemma. 
1.4. Action of nilpotent Lie groups on a simply connected homogeneous spaces.

Let $\mathscr{B}: X_{1}, \ldots, X_{m}$ be a Jordan-Hölder basis in $\mathfrak{g}$, let $\mathfrak{h}$ be a subalgebra of $\mathfrak{g}$, and set $H=\exp \mathfrak{h}$. Define the set $e=\left\{1 \leq j \leq m \mid X_{j} \notin \mathfrak{g}_{j-1}+\mathfrak{h}\right\}$, and write $e=\left\{j_{1}<\cdots<j_{d}\right\}$. Then $X_{j_{1}}, \ldots, X_{j_{d}}$ is a basis for $\mathfrak{g}(\bmod \mathfrak{h})$ of subalgebra type. In fact, set $\mathfrak{h}_{0}=\mathfrak{h}$, and set $\mathfrak{h}_{r}=\mathbf{R} X_{j_{r}} \oplus \cdots \oplus \mathbf{R} X_{j_{1}} \oplus \mathfrak{h}$ for $1 \leq r \leq d$. Clearly we have $\mathfrak{g}_{j}+\mathfrak{h}=\mathfrak{h}_{r}$ for $j_{r} \leq j<j_{r+1}$. But then $\left[\mathfrak{h}_{r}, \mathfrak{h}_{r-1}\right]=\left[\mathfrak{g}_{j_{r}}+\mathfrak{h}, \mathfrak{g}_{j_{r-1}}+\mathfrak{h}\right] \subset\left[\mathfrak{g}, \mathfrak{g}_{j_{r}}\right]+[\mathfrak{h}, \mathfrak{h}] \subset \mathfrak{g}_{j_{r}-1}+\mathfrak{h}=\mathfrak{h}_{r-1}$, and this shows the claim. It follows that the map $\sigma_{G / H}^{\mathscr{B}}: G / H \rightarrow \mathbf{R}^{d}$ given by

$$
\sigma_{G / H}^{\mathscr{B}}: \exp t_{d} X_{j_{d}} \cdots \exp t_{1} X_{j_{1}} H \rightarrow\left(t_{1}, \ldots, t_{d}\right)
$$

is a polynomial chart. We shall call $\sigma_{G / H}^{\mathscr{B}}$ for the $\mathscr{B}$-chart of $G / H$.

For $x \in \mathbf{R}^{m}$ and $u \in \mathbf{R}^{d}$ we write $x \cdot u=\sigma_{G / H}^{\mathscr{B}}\left(\left(\sigma_{G}^{\mathscr{B}}\right)^{-1}(x)\left(\sigma_{G / H}^{\mathscr{B}}\right)^{-1}(u)\right)$. In other words, we identify $G$ with $\mathbf{R}^{m}$ via $\sigma_{G}^{\mathscr{B}}$ and $G / H$ with $\mathbf{R}^{d}$ via $\sigma_{G / H}^{\mathscr{B}}$ and transport the action of $G$ on $G / H$ to a smooth action of $\mathbf{R}^{m}$ on $\mathbf{R}^{d}$. With this notation we have

Lemma 1.4.1. The function $Q(x, u)=x \cdot u$ is a polynomial function on $\mathbf{R}^{m} \times \mathbf{R}^{d}$, and writing $Q(x, u)=\left(Q_{1}(x, u), \ldots, Q_{d}(x, u)\right)$ we have

$$
Q_{r}(x, u)=x_{j_{r}}+u_{r}+\bar{Q}_{r}\left(x_{j_{r}+1}, \ldots, x_{m}, u_{r+1}, \ldots, u_{d}\right),
$$

for $1 \leq r \leq d$, where we have written $x=\left(x_{1}, \ldots, x_{m}\right), u=\left(u_{1}, \ldots, u_{d}\right)$, and where $\bar{Q}_{r}$ are polynomial functions with properties as indicated.

Proof. If $j \notin e$ there exists $X_{j}^{\prime} \in \mathfrak{h}$ such that $X_{j}-X_{j}^{\prime} \in \mathfrak{g}_{j-1}$. Using Lemma 1.2.5 we see that it is no loss of generality to assume that $X_{j} \in \mathfrak{h}$ for all $j \notin e$. For $u \in \mathbf{R}^{d}$ we then set $y_{j_{r}}=u_{r}$ for $r=1, \ldots, d$ and $y_{j}=0$ for $j \notin e$, and we set $z=x \cdot y$. Then with the notation from Lemma 1.2.4 we have that $(x \cdot u)_{r}=$ $S_{j_{r}}(z)$, i.e., $Q_{r}(x, u)=S_{j_{r}}(z)=x_{j_{r}}+y_{j_{r}}+\bar{S}_{j_{r}}\left(x_{j_{r}+1}, \ldots, x_{m}, y_{j_{r}+1}, \ldots, y_{m}\right)=$ $x_{j_{r}}+u_{r}+\bar{Q}_{r}\left(x_{j_{r}+1}, \ldots, x_{m}, u_{r+1}, \ldots, u_{d}\right)$. This ends the proof of the lemma.

Corollary 1.4.2. The map $(s, a) \rightarrow s a: G \times G / H \rightarrow G / H$ is a polynomial map.

1.5. Polynomial vector fields on simply connected homogeneous spaces of nilpotent Lie groups. Let $\mathfrak{h}$ be a subalgebra in $\mathfrak{g}$, and set $H=\exp \mathfrak{h}$. For $X \in \mathfrak{g}$ we define the vector field $\tilde{X}$ on $G / H$ as follows:

$$
\tilde{X} f(a)=\left.\frac{d}{d v} f(\exp -v X a)\right|_{v=0},
$$

$a \in G / H, f \in \mathscr{E}(G / H)$. It follows from Corollary 1.4.2 that $\tilde{X}$ is a polynomial vector field on $G / H$.

Let now $\mathscr{B}: X_{1}, \ldots, X_{m}$ be a Jordan-Hölder basis in $\mathfrak{g}$, set $e=\{1 \leq j \leq$ $\left.m \mid X_{j} \notin \mathfrak{g}_{j-1}+\mathfrak{h}\right\}$, and write $e=\left\{k_{1}<\cdots<k_{d / 2}\right\}$. 
Lemma 1.5.1. The space of polynomial vector fields $\mathscr{U}(G / H)$ on $G / H$ is a free $\mathscr{P}(G / H)$-module with basis $\tilde{X}_{k_{1}}, \ldots, \tilde{X}_{k_{d / 2}}$.

Proof. We consider the $\mathscr{B}$-chart $\sigma_{G / H}^{\mathscr{B}}$ of $G / H$ given by

$$
\sigma_{G / H}^{\mathscr{B}}: \exp t_{d / 2} X_{k_{d / 2}} \cdots \exp t_{1} X_{k_{1}} \rightarrow\left(t_{1}, \ldots, t_{d / 2}\right),
$$

see $\S 1.4$. Using this chart we identify $G / H$ with $\mathbf{R}^{d / 2}$ and we can think of $\tilde{X}_{j}$ as polynomial vector fields on $\mathbf{R}^{d / 2}$. Similarly we identify $G$ with $\mathbf{R}^{m}$ via the $\mathscr{B}$-chart $\sigma_{G}^{\mathscr{B}}$, see $\S 1.1$. Let us then compute $\tilde{X}_{k_{s}}, 1 \leq s \leq d / 2$. By Lemma 1.4.1 the action of $G=\mathbf{R}^{m}$ on $G / H=\mathbf{R}^{d / 2}$ is given by $x \cdot u=Q(x, u)$, $x \in \mathbf{R}^{m}, u \in \mathbf{R}^{d / 2}$, where $Q=\left(Q_{1}, \ldots, Q_{d / 2}\right)$ is a polynomial function such that $Q_{r}(x, u)=x_{k_{r}}+u_{r}+\bar{Q}_{r}\left(x_{k_{r}+1}, \ldots, x_{m}, u_{r+1}, \ldots, u_{d / 2}\right)$. Setting

$$
a_{r s}(u)=\left.\frac{d}{d v} Q_{s}\left(\exp -v X_{k_{r}} \cdot u\right)\right|_{v=0}
$$

for $1 \leq r, s \leq d / 2, u \in \mathbf{R}^{d / 2}$, we get, using the chain rule:

$$
\begin{aligned}
\tilde{X}_{k_{r}} f(u) & =\left.\frac{d}{d v} f\left(\exp -v X_{k_{r}} \cdot u\right)\right|_{v=0} \\
& =\left.\frac{d}{d v} f\left(Q\left(\exp -v X_{k_{r}}, u\right)\right)\right|_{v=0}=\sum_{s=1}^{d / 2} \frac{\partial f}{\partial u_{s}}(u) a_{r s}(u) .
\end{aligned}
$$

Now

$$
Q_{s}\left(\exp -v X_{k_{r}}, u\right)=\left\{\begin{array}{l}
u_{s} \text { for } s>r \\
u_{s}-v+\bar{Q}_{s}\left(\exp -v X_{k_{r}}, u_{s+1}, \ldots, u_{m}\right) \quad \text { for } r=s, \\
u_{s}+\bar{Q}_{s}\left(\exp -v X_{k_{r}}, u_{s+1}, \ldots, u_{m}\right) \text { for } r<s .
\end{array}\right.
$$

This implies that

$$
a_{r s}(u)=\left\{\begin{array}{l}
0 \text { for } s>r \\
-1 \text { for } s=r \\
a_{r s}\left(u_{s+1}, \ldots, u_{n}\right) \text { for } s<r .
\end{array}\right.
$$

It follows that the martix $\mathbf{A}(u)=\left[a_{r s}\right]_{1 \leq r, s \leq d / 2}$ is a lower triangular matrix with polynomial entries and with -1 in the diagonal. But then also the inverse matrix $\mathbf{B}(u)=\mathbf{A}(u)^{-1}$ is of the same kind. Now writing $\mathbf{B}(u)=\left[b_{r s}\right]_{1 \leq r, s \leq d / 2}$ we have

$$
\frac{\partial}{\partial u_{r}}=\sum_{s=1}^{d / 2} b_{r s}(u) \tilde{X}_{k_{s}} .
$$

It follows that $\tilde{X}_{k_{1}}, \ldots, \tilde{X}_{k_{d / 2}}$ generates $\mathscr{U}(G / H)$ as a $\mathscr{P}(G / H)$-module. That $\tilde{X}_{k_{1}}, \ldots, \tilde{X}_{k_{d / 2}}$ is actually a basis for $\mathscr{U}(G / H)$ follows immediately from the fact that the matrix $\mathbf{A}(u)$ is regular. This ends the proof of the lemma. 
For $s \in G$ define the endomorphism $\gamma_{G / H}(s)$ of $\mathscr{E}(G / H)$ by $\gamma_{G / H}(s) f(a)=$ $f\left(s^{-1} a\right), a \in G / H$. Then $s \rightarrow \gamma_{G / H}(s)$ is a differentiable representation of $G$. For $X \in \mathfrak{g}$ and $f \in \mathscr{E}(G / H)$ we have $d \gamma_{G / H}(X) f=\tilde{X} f$. Let $I$ denote the identity operator.

Lemma 1.5.2. The space $\mathscr{A}^{1}(G / H)$ is a free $\mathscr{P}(G / H)$-module with basis $I$, $d \gamma_{G / H}\left(X_{k_{1}}\right), \ldots, d \gamma_{G / H}\left(X_{k_{d / 2}}\right)$.

Proof. Let $D \in \mathscr{A}^{1}(G / H)$. Since there exists a bi-polynomial diffeomorphism from $G / H$ to $\mathbf{R}^{d}, D$ can be written uniquely as $D f=\xi(f)+\varphi_{0} f$, where $\xi$ is a polynomial vector field on $G / H$ and $\varphi_{0} \in \mathscr{P}(G / H)$. The lemma now follows from Lemma 1.5.1.

1.6. The polynomial structure on coadjoint orbits of nilpotent Lie groups. Let $G$, $\mathfrak{g}$ be as before, and let $O=G g$ be the coadjoint orbit through $g \in \mathfrak{g}^{*}$. The orbit $O$ is equipped with the smooth structure it gets from the identification $G / G_{g} \simeq O$. We also carry over the polynomial structure already defined on $G / G_{g}$ to $O$. It is easily seen that this definition of a polynomial structure on $O$ is independent of the choice of $g \in O$ (Corollary 1.4.2).

Given a Jordan-Hölder basis $\mathscr{B}: X_{1}, \ldots, X_{m}$ in $\mathfrak{g}$ there exist indices $j_{1}<$ $\cdots<j_{d}$ (the "jump indices"of $O$ relative to $\mathscr{B}$ ) and polynomial functions $R_{1}, \ldots, R_{m}$ in the variables $\left(y_{j_{1}}, \ldots, y_{j_{d}}\right)$ such that $R_{j_{u}}(y)=y_{j_{u}}, 1 \leq u \leq d$, and such that $O$ can be described as follows

$$
O=\left\{l=\sum_{j=1}^{m} R_{j}\left(y_{j_{1}}, \ldots, y_{j_{d}}\right) l_{j} \mid\left(y_{j_{1}}, \ldots, y_{j_{d}}\right) \in \mathbf{R}^{d}\right\},
$$

where $l_{1}, \ldots, l_{m}$ is the basis in $\mathfrak{g}^{*}$ dual to $X_{1}, \ldots, X_{m}$. The set of jump indices $J_{g}$ associated with $g \in \mathfrak{g}^{*}$ can be found as

$$
J_{g}=\left\{1 \leq j \leq m \mid \mathfrak{g}_{j} \not \subset \mathfrak{g}_{j-1}+\mathfrak{g}_{g}\right\},
$$

cf. $\S$ 4.2. Define the map $\sigma_{O}^{\mathscr{B}}: O \rightarrow \mathbf{R}^{d}$ by

$$
\sigma_{O}^{\mathscr{B}}: l=\sum_{j=1}^{m} R_{j}\left(y_{j_{1}}, \ldots, y_{j_{d}}\right) l_{j} \rightarrow\left(y_{j_{1}}, \ldots, y_{j_{d}}\right)=\left(\left\langle l, X_{j_{1}}\right\rangle, \ldots,\left\langle l, X_{j_{d}}\right\rangle\right) .
$$

Lemma 1.6.1. The map $\sigma_{O}^{\mathscr{B}}$ is a polynomial chart on $O$.

Proof. Let $g \in O$. It follows from [10, p. 265] that there exists a basis $Y_{d}, \ldots, Y_{1}$ of $\mathfrak{g}\left(\bmod \mathfrak{g}_{g}\right)$ of subalgebra type such that if we write

$$
\exp t_{1} Y_{1} \cdots \exp t_{d} Y_{d} g=\sum_{j=1}^{m} Q_{j}(t) l_{j}
$$

where $t=\left(t_{1}, \ldots, t_{d}\right)$, then the functions $Q_{j}(t)$ are polynomial functions with the following properties: For $j_{r} \leq j<j_{r+1}$ we have

$$
Q_{j}(t)=Q_{j}\left(t_{1}, \ldots, t_{r}, 0, \ldots, 0\right)
$$


and

$$
Q_{j_{r}}(t)=t_{r}+Q_{j_{r}}\left(t_{1}, \ldots, t_{r-1}, 0, \ldots, 0\right) .
$$

Let now $\sigma$ be the polynomial chart associated with $Y_{d}, \ldots, Y_{1}$. For $l \in O$, write $\sigma(l)=t=\left(t_{1}, \ldots, t_{d}\right)$ and $\sigma_{O}^{\mathscr{B}}(l)=y=\left(y_{1}, \ldots, y_{d}\right)$. Then

$$
\begin{aligned}
& y_{1}=t_{1}+Q_{j_{1}}(0, \ldots, 0) \\
& y_{2}=t_{2}+Q_{j_{2}}\left(t_{1}, 0, \ldots, 0\right) \\
& y_{3}=t_{3}+Q_{j_{3}}\left(t_{1}, t_{2}, 0, \ldots, 0\right) \\
& \quad \vdots \\
& y_{d}=t_{d}+Q_{j_{d}}\left(t_{1}, \ldots, t_{d-1}, 0\right) .
\end{aligned}
$$

From this it follows immediately that $\sigma_{O}^{\mathscr{B}}$ is a polynomial chart.

Corollary 1.6.2. A function $f: O \rightarrow \mathbf{C}$ is polynomial if and only if it is the restriction to $O$ of a polynomial function on $\mathfrak{g}^{*}$.

Proof. If $\varphi \in \mathscr{P}(O)$, then $f=\varphi \circ\left(\sigma_{O}^{\mathscr{B}}\right)^{-1}$ is a polynomial function on $\mathbf{R}^{d}$, and $\varphi$ is the restriction to $O$ of the function $l \rightarrow f\left(\left\langle l, X_{j_{1}}\right\rangle, \ldots,\left\langle l, X_{j_{d}}\right\rangle\right)$ on $\mathfrak{g}^{*}$. If $\varphi \in \mathscr{P}\left(\mathfrak{g}^{*}\right)$ we can write $\varphi=f\left(\left\langle X_{1}, l\right\rangle, \ldots,\left\langle X_{m}, l\right\rangle\right)$, where $f$ is a polynomial function on $\mathbf{R}^{m}$. But then $\varphi \circ\left(\sigma_{O}^{\mathscr{B}}\right)^{-1}(y)=f\left(R_{1}(y), \ldots, R_{m}(y)\right)$. Since $R_{1}, \ldots, R_{m}$ are polynomial functions it follows that the restriction of $\varphi$ to $O$ is a polynomial function.

1.7. Rapidly decreasing sections and polynomial coefficient differential operators in homogeneous line bundles of nilpotent Lie groups. Let $\mathfrak{h}$ be a subalgebra of $\mathfrak{g}$, and let $H=\exp \mathfrak{h}$. Let $Y_{1}, \ldots, Y_{n}$ be a basis of $\mathfrak{g}(\bmod \mathfrak{h})$ of subalgebra type, and let $\sigma: G / H \rightarrow \mathbf{R}^{n}$ given by

$$
\sigma: \exp t_{n} Y_{n} \cdots \exp t_{1} Y_{1} H \rightarrow\left(t_{1}, \ldots, t_{n}\right)
$$

be the corresponding chart on $G / H$. We define $c: G \rightarrow G / H$ to be the quotient map and $\kappa: G / H \rightarrow G$ to be the map

$$
\kappa: \exp t_{n} Y_{n} \cdots \exp t_{1} Y_{1} H \rightarrow \exp t_{n} Y_{n} \cdots \exp t_{1} Y_{1} .
$$

In this way $\kappa$ is a cross-section for $c: c \circ \kappa=\mathrm{id}_{G / H}$. We also define the map $\theta: \mathbf{R}^{n} \rightarrow G$ by

$$
\theta:\left(t_{1}, \ldots, t_{n}\right) \rightarrow \exp t_{n} Y_{n} \cdots \exp t_{1} Y_{1} .
$$

The maps $c, \kappa$ and $\theta$ are polynomial maps, and we have

$$
\theta \circ \sigma=\kappa \text {. }
$$

Define the map $a: G \rightarrow H$ by $s=\kappa(c(s)) a(s)$ for all $s \in G$, i.e., $a(s)=$ $(\kappa(c(s)))^{-1} s$. Then $a$ is a polynomial map, and $a(s h)=a(s) h$ for all $s \in G$, $h \in H$. We also define the map $A: G \rightarrow \mathfrak{h}$ by $A(s)=\log (a(s))$. Clearly $A$ is also a polynomial map. 
Suppose now that $\chi: H \rightarrow \mathbf{T}$ is a unitary character, and let $g \in \mathfrak{g}^{*}$ be such that $\chi(\exp X)=e^{i\langle g, X\rangle}$ for all $X \in \mathfrak{h}$. In [9] we have defined $\mathscr{E}(G, \chi)$ to be the set of all smooth functions $f$ on $G$ such that $f(s h)=\chi(h)^{-1} f(s)$ for all $s \in G, h \in H$. We define a linear bijection $V: \mathscr{E}(G, \chi) \rightarrow \mathscr{E}\left(\mathbf{R}^{n}\right)$ by

$$
(V f)(t)=f(\theta(t)), \quad f \in \mathscr{E}(G, \chi), t \in \mathbf{R}^{n} .
$$

The inverse of $V$ is given by

$$
V^{-1} h(s)=e^{i\langle g, A(s)\rangle} h(\sigma(c(s))), \quad h \in \mathscr{E}\left(\mathbf{R}^{n}\right), s \in G .
$$

Denote by $\mathscr{S}\left(\mathbf{R}^{n}\right)$ the space of rapidly decreasing functions on $\mathbf{R}^{n}$ (the Schwartz functions) and let $\mathscr{A}^{k}\left(\mathbf{R}^{n}\right)$ denote the space of differential operators on $\mathbf{R}^{n}$ of order at most $k$ and having polynomial coefficients, and set $\mathscr{A}\left(\mathbf{R}^{n}\right)=$ $\bigcup_{k=0}^{\infty} \mathscr{A}^{k}\left(\mathbf{R}^{n}\right)$ (the Weyl algebra).

Definition 1.7.1. We define $\mathscr{S}(G, \chi)$ to be the inverse image by $V$ of $\mathscr{S}\left(\mathbf{R}^{n}\right)$, and we define $\mathscr{A}(G, \chi)\left(\mathscr{A}^{k}(G, \chi)\right)$ to be the transport of $\mathscr{A}\left(\mathbf{R}^{n}\right)\left(\mathscr{A}^{k}\left(\mathbf{R}^{n}\right)\right.$, $k=0,1, \ldots)$ by $V^{-1}$.

Lemma 1.7.2. The definition of $\mathscr{S}(G, \chi)$ and $\mathscr{A}^{k}(G, \chi)$ is independent of the choice of basis of $\mathfrak{g}$ (mod $\mathfrak{h})$ of subalgebra type.

Proof. Suppose that $Y_{1}^{\prime}, \ldots, Y_{n}^{\prime}$ is another basis of $\mathfrak{g}(\bmod \mathfrak{h})$ of subalgebra type. The objects defined relative to this basis are all marked with a prime. We define the linear bijection $W: \mathscr{E}\left(\mathbf{R}^{n}\right) \rightarrow \mathscr{E}\left(\mathbf{R}^{n}\right)$ by $W=V^{\prime} V^{-1}$. We have to show that $W$ maps $\mathscr{S}\left(\mathbf{R}^{n}\right)$ onto $\mathscr{S}\left(\mathbf{R}^{n}\right)$, and that $W$ transports $\mathscr{A}^{k}\left(\mathbf{R}^{n}\right)$ onto $\mathscr{A}^{k}\left(\mathbf{R}^{n}\right)$. We calculate $W$ :

$$
(W h)(t)=e^{-i\left\langle g, A\left(\theta^{\prime}(t)\right)\right\rangle} h\left(\sigma \circ \sigma^{\prime-1}(t)\right), \quad h \in \mathscr{E}\left(\mathbf{R}^{n}\right), t \in \mathbf{R}^{n} .
$$

Set $\varphi=\sigma \circ \sigma^{\prime-1}$, and set $S(t)=-\left\langle g, A\left(\theta^{\prime}(t)\right)\right\rangle, t \in \mathbf{R}^{n}$. Then $\varphi$ is a bipolynomial diffeomorphism, and $S$ is a real-valued polynomial map, and we have with this notation:

$$
(W h)(t)=e^{i S(t)} h(\varphi(t)), \quad h \in \mathscr{E}\left(\mathbf{R}^{n}\right), \quad t \in \mathbf{R}^{n} .
$$

Similarly, if we define $\psi=\varphi^{-1}=\sigma^{\prime} \circ \sigma^{-1}$, and $T(t)=-\left\langle g, A^{\prime}(\theta(t))\right\rangle$ we have

$$
\left(W^{-1} h\right)(t)=e^{i T(t)} h(\psi(t)), \quad h \in \mathscr{E}\left(\mathbf{R}^{n}\right), t \in \mathbf{R}^{n} .
$$

If $p$ is a polynomial function on $\mathbf{R}^{n}$ we let $M_{p}$ denote the multiplication operator on $\mathscr{E}\left(\mathbf{R}^{n}\right)$ defined by $p$. One easily calculates

$$
W M_{p} W^{-1}=M_{p \circ \varphi} \text {. }
$$

Moreover, if $D_{j}$ denotes the differential operator $\partial / \partial t_{j}$ for $1 \leq j \leq n$, one easily calculates that

$$
W D_{j} W^{-1}=\sum_{k=1}^{n} M_{\partial \varphi_{k} / \partial t_{j} \circ \varphi} D_{k}+M_{\partial T / \partial t_{j} \circ \varphi}
$$


From this we see immediately that $W$ transports $k$ th order differential operators with polynomial coefficients on $\mathbf{R}^{n}$ onto itself, and from this it follows that $W$ carries $\mathscr{S}\left(\mathbf{R}^{n}\right)$ onto itself. This ends the proof of the lemma.

For $s \in G$ we define the endomorphism $\gamma_{\chi}(s)$ of $\mathscr{E}(G, \chi)$ by $\gamma_{\chi}(s) f(t)=$ $f\left(s^{-1} t\right), t \in G$. The map $s \rightarrow \gamma_{\chi}(s)$ is a differentiable representation of $G$.

Lemma 1.7.3. We have that $d \gamma_{\chi}(X) \in \mathscr{A}^{1}(G, \chi)$ for each $X \in \mathfrak{g}$.

Proof. We define a linear isomorphism $T: \mathscr{E}(G, \chi) \rightarrow \mathscr{E}(G / H)$ as follows:

$$
(T f)(a)=f(\kappa(a)), \quad f \in \mathscr{E}(G, \chi), a \in G / H .
$$

The inverse is given by

$$
\left(T^{-1} h\right)(s)=e^{-i\langle g, A(s)\rangle} h(c(s)), \quad h \in \mathscr{E}(G / H) .
$$

Clearly $T$ is a linear isomorphism. When we transport $\mathscr{A}^{1}(G, \chi)$ to $\mathscr{E}(G / H)$ by $T$ we get $\mathscr{A}^{1}(G / H)$ by Definition 1.7.1. We then calculate $T d \gamma_{\chi}(X) T^{-1}$. We get for $h \in \mathscr{E}(G / H), x \in G / H$,

$$
\begin{aligned}
T d \gamma_{\chi}(X) T^{-1} h(x) & \left.=d \gamma_{\chi}(X) T^{-1} h(\kappa(x))=\frac{d}{d v} T^{-1} h(\exp -v X \kappa(x))\right)\left.\right|_{v=0} \\
& =\left.\frac{d}{d v} e^{-i\langle g, A(\exp -v X \kappa(x))\rangle} h(\exp -v X x)\right|_{v=0} \\
& =\left.\frac{d}{d v} e^{-i\langle g, A(\exp -v X \kappa(x))\rangle}\right|_{v=0} h(x)+d \gamma_{G / H}(X) h(x) .
\end{aligned}
$$

Therefore, if we set

$$
\alpha_{X}(a)=\left.\frac{d}{d v} e^{-i\langle g, A(\exp -v X \kappa(x))\rangle}\right|_{v=0}=-\left.i \frac{d}{d v}\langle g, A(\exp -v X \kappa(x))\rangle\right|_{v=0}
$$

we have that $\alpha_{X} \in \mathscr{P}(G / H)$, and

$$
T d \gamma_{\chi}(X) T^{-1} h=d \gamma_{G / H}(X) h+\alpha_{X} h
$$

for $h \in \mathscr{E}(G / H)$. This proves the lemma, since $d \gamma_{G / H}(X) \in \mathscr{A}^{1}(G / H)$, see $\S 1.5$.

Proposition 1.7.4. The space $\mathscr{A}^{1}(G, \chi)$ is a free $\mathscr{P}(G / H)$-module with basis $I, d \gamma_{\chi}\left(X_{k_{1}}\right), \ldots, d \gamma_{\chi}\left(X_{k_{d / 2}}\right)$.

Proof. The lemma says that each element $D \in \mathscr{A}^{1}(G, \chi)$ can be written uniquely

$$
D=\sum_{u=1}^{d / 2} \varphi_{u} d \gamma_{\chi}\left(X_{k_{u}}\right)+\varphi_{0}
$$

where $\varphi_{0}, \varphi_{1}, \ldots, \varphi_{d / 2}$ are in $\mathscr{P}(G / H)$. Transforming this relation by the operator $T$ from the previous lemma we get

$$
T D T^{-1}=\sum_{u=1}^{d / 2} \varphi_{u} d \gamma_{G / H}\left(X_{k_{u}}\right)+\varphi_{0}^{\prime},
$$

where $\varphi_{0}^{\prime}=\sum_{u=1}^{d / 2} \varphi_{u} \alpha_{X_{k_{u}}}+\varphi_{0}$. The result now follows from Lemma 1.5.2. 
1.8. Realizing representations. Let $g \in \mathfrak{g}^{*}$ and let $\mathfrak{h}$ be a real polarization at $g$. Set $H==\exp \mathfrak{h}$, and let $\chi$ be the unitary character of $H$ given by $\chi(\exp X)=$ $e^{i\langle g, X\rangle}, X \in \mathfrak{h}$. The representation $\pi_{\chi}=\operatorname{ind}_{H \dagger G} \chi$ of $G$ is the irreducible representation associated with the orbit $O=G g$. The representation space of $\pi_{\chi}$ is $L^{2}(G, \chi)$. Let now $X_{1}, \ldots, X_{d / 2}$ be a basis for $\mathfrak{g}(\bmod \mathfrak{h})$ of subalgebra type. Using the notation from the previous section we can define a unitary map $V: L^{2}(G, \chi) \rightarrow L^{2}\left(\mathbf{R}^{d / 2}\right)$ using the same formula for $V$ as before. Let us then define the representation $\pi_{\chi}^{V}$ of $G$ on $L^{2}\left(\mathbf{R}^{d / 2}\right)$ by

$$
\pi_{\chi}^{V}(s)=V \pi_{\chi}(s) V^{-1} \text {. }
$$

Let $b: G \times \mathbf{R}^{d / 2} \rightarrow H$ be the polynomial function defined by $b(s, t)=$ $a\left(s^{-1} \theta(t)\right), s \in G, t \in \mathbf{R}^{d / 2}$, and define the polynomial function $B: G \times \mathbf{R}^{d / 2} \rightarrow$ $\mathfrak{h}$ by $\exp B(s, t)=b(s, t), s \in G, t \in \mathbf{R}^{d / 2}$. Further, define a polynomial action of $G$ in $\mathbf{R}^{d / 2}$ by transport of structure (i.e., $\left.s t=\sigma\left(s \sigma^{-1}(t)\right)\right)$. The representation $\pi_{\chi}^{V}$ is equivalent to $\pi_{\chi}$, and we have

$$
\pi_{\chi}^{V}(s) h(t)=\chi(b(s, t))^{-1} h\left(s^{-1} t\right)=e^{-i\langle g, B(s, t)\rangle} h\left(s^{-1} t\right),
$$

$h \in L^{2}\left(\mathbf{R}^{d / 2}\right), s \in G, t \in \mathbf{R}^{d / 2}$.

\section{Orbits, Poisson STRUCTURe AND QUANTIZATION OF NILPOTENT LIE GROUPS}

In a recent paper [9] I have obtained certain results about the canonical symplectic structure of connected Lie groups, and in particular of exponential solvable Lie groups. In this section we shall briefly specialize to the nilpotent case, where we obtain more detailed results.

Let $G$ be a connected, simply connected nilpotent Lie group with Lie algebra $\mathfrak{g}$, and let $O$ be a coadjoint orbit of $G$.

2.1. Polynomial functions and Poisson bracket. First we consider how polynomial functions on $O$ behave under Poisson bracket.

We select a Jordan-Hölder sequence

$$
\mathscr{F}: \mathfrak{g}=\mathfrak{g}_{m} \supset \mathfrak{g}_{m-1} \supset \cdots \supset \mathfrak{g}_{1} \supset \mathfrak{g}_{0}=\{0\}
$$

and a compatible Jordan-Hölder basis $\mathscr{B}: X_{1}, \ldots, X_{m}$, i.e. $X_{j} \in \mathfrak{g}_{j} \backslash \mathfrak{g}_{j-1}$, $j=1, \ldots, m$.

We set

$$
e=\left\{1 \leq j \leq m \mid X_{j} \notin \mathfrak{g}_{j-1}+\mathfrak{g}_{g}\right\}
$$

for some fixed element $g \in O$, and write $e=\left\{j_{1}<\cdots<j_{d}\right\}$, where $d$ is the dimension of the orbit. Let $\sigma_{O}^{\mathscr{B}}$ be the $\mathscr{B}$-chart of $O$, i.e., the chart defined by $\sigma_{O}^{\mathscr{B}}(l)=\left(\left\langle l, X_{j_{1}}\right\rangle, \ldots,\left\langle l, X_{j_{d}}\right\rangle\right)$, see $\S 1.6$. We then know that the functions $R_{j}=\psi^{X_{j}} \circ\left(\sigma_{O}^{\mathscr{B}}\right)^{-1}$ are polynomial functions on $\mathbf{R}^{d}$ with $R_{j_{r}}(y)=y_{r}$, 
$r=1, \ldots, d$, where $y=\left(y_{1}, \ldots, y_{d}\right)$. Writing $\sigma_{O}^{\mathscr{B}}=\sigma=\left(y_{1}, \ldots, y_{d}\right)$ we have that the matrix $\mathbf{M}_{\sigma}(l)=\left[\left\{y_{u}, y_{v}\right\}(l)\right]_{1 \leq u, v \leq d}$ is equal to the matrix $\mathbf{M}_{e}(l)=\left[\left\langle l,\left[X_{j_{u}}, X_{j_{v}}\right]\right\rangle\right]_{1 \leq u, v \leq d}\left[9\right.$, Lemma 1.1.8]. Let now $c_{i j k}$ be the structure constants of $\mathfrak{g}$ associated with the basis $\mathscr{B}$, i.e., $\left[X_{i}, X_{j}\right]=\sum_{k=1}^{m} c_{i j k} X_{k}$. The entries of the matrix $\mathbf{M}_{\sigma}(l)$ have the form $\sum_{k=1}^{m} c_{j_{u} j_{v} k} \psi^{X_{k}}(l)$, and therefore the entries $a_{u v}(y)$ of the matrix $\mathbf{M}_{\sigma}\left(\sigma^{-1}(y)\right)$ are of the form

$$
a_{u v}(y)=\sum_{k=1}^{m} c_{j_{u} j_{v}} R_{k}(y)
$$

in particular these entries are polynomial functions. We can now write down the Hamiltonian vector fields and the Poisson bracket in these coordinates:

$$
\begin{gathered}
\xi_{\varphi}=\sum_{u=1}^{d}\left(\sum_{v=1}^{d} a_{u v} \frac{\partial \varphi}{\partial y_{v}}\right) \frac{\partial}{\partial y_{u}}, \\
\{\varphi, \psi\}=\sum_{u, v=1}^{d} a_{u v} \frac{\partial \varphi}{\partial y_{u}} \frac{\partial \psi}{\partial y_{v}}
\end{gathered}
$$

for $\varphi, \psi \in \mathscr{E}(O)$. From these remarks we derive the following lemma:

Lemma 2.1.1. For each $\varphi \in \mathscr{P}(O)$ the vector field $\xi_{\varphi}$ is polynomial. Moreover, the space $\mathscr{P}(O)$ is closed under Poisson bracket.

Let us also note that with the notation from $[9, \S 1.1]$ we have that the matrix $\boldsymbol{\Omega}_{\sigma}(l)=\left[\omega_{l}\left(\left(\partial / \partial y_{u}\right)_{l},\left(\partial / \partial y_{v}\right)_{l}\right)\right]_{1 \leq u, v \leq d}$ is equal to $-\mathbf{M}_{\sigma}^{-1}(l)$. Since the determinant $Q_{e}(l)$ of $\mathbf{M}_{e}(l)$ is constant on the orbit, [7, Lemma 1.1.1, $\mathbf{p}$. 427] (see also $\S 4.2$ ), we see that also $\boldsymbol{\Omega}_{\sigma}\left(\sigma^{-1}(y)\right)$ has polynomial entries. We therefore get

Lemma 2.1.2. The canonical symplectic structure $\omega_{O}$ is a polynomial 2-form.

2.2. Polynomial quantizable functions. Next we shall consider the structure of the space of polynomial quantizable functions associated with a given real polarization. The main result is Proposition 2.2.9.

Let $g \in O$ and let $\mathfrak{h}$ be a real polarization at $g$. This means that $\mathfrak{h}$ is a subalgebra of $\mathfrak{g}$ and that $\mathfrak{h}$ is maximal isotropic with respect to $B_{g}$. Let $H$ be the analytic subgroup of $G$ corresponding to $\mathfrak{h}$.

For $l=s g \in O$ we set $\mathfrak{h}_{l}=\operatorname{Ad}(s) \mathfrak{h}$ and $H_{l}=s H s^{-1}$. Further, we let $\alpha_{l}: \mathfrak{g} / \mathfrak{g}_{l} \rightarrow T_{l}(O)$ denote the canonical vector space isomorphism and we set $F_{l}$ to be the image of $\mathfrak{h}_{l} / g_{l}$ by $\alpha_{l}$. We have $F_{g}=\alpha_{g}\left(\mathfrak{h} / \mathfrak{g}_{g}\right)$, and denoting by $\gamma(s): O \rightarrow O$ the map $\gamma(s): l \rightarrow s l$ we have $\gamma(s)_{*} F_{l}=F_{s l}$. The map $F: l \rightarrow F_{l}$ is a $G$-invariant real polarization of the symplectic manifold $(O, \omega)$ (cf. [9]).

The group $H$ defines a fibration of the orbit $O \simeq G / G_{g}$ with base $G / H$ and with the projection given by $\pi: l=s g \rightarrow s H$. The fiber through $l=s g$ 
is $\mathscr{L}(l)=\{$ shg $\mid h \in H\}=l+\mathfrak{h}_{l}^{\perp}$, since $\mathfrak{h}$ satisfies the Pukanszky condition [10]. The vertical subspace through $l \in O$ is $F_{l}$.

Let $\mathscr{V}(O)$ denote the set of all smooth complex vector fields on $O$. In [9] we have defined certain Lie subalgebras $\mathscr{V}_{F}^{0}(O)$ and $\mathscr{V}_{F}^{1}(O)$ of $\mathscr{V}(O)$, and we have defined certain Lie subalgebras $\mathscr{E}_{F}^{0}(O)$ and $\mathscr{E}_{F}^{1}(O)$ of $\mathscr{E}(O)$ (equipped with Poisson bracket). We set $\mathscr{P}_{F}^{0}(O)=\mathscr{E}_{F}^{0}(O) \cap \mathscr{P}(O)$ and $\mathscr{P}_{F}^{1}(O)=\mathscr{E}_{F}^{1}(O) \cap$ $\mathscr{P}(O)$.

It follows from [9] that the map from $\mathscr{E}(G / H)$ to $\mathscr{E}_{F}^{0}(O)$ given by $\varphi \rightarrow \varphi \circ \pi$ is a linear bijection. Considering polynomial functions we have

Lemma 2.2.1. The map $\varphi \rightarrow \varphi \circ \pi$ maps $\mathscr{P}(G / H)$ onto $\mathscr{P}_{F}^{0}(O)$.

Proof. This follows from Lemma 1.3.2.

We now set

$$
e=\left\{1 \leq j \leq m \mid X_{j} \notin \mathfrak{g}_{j-1}+\mathfrak{h}\right\},
$$

and write $e=\left\{k_{1}<\cdots<k_{d / 2}\right\}$.

Lemma 2.2.2. For any $l \in O$ we have that

$$
e=\left\{1 \leq j \leq m \mid X_{j} \notin \mathfrak{g}_{j-1}+\mathfrak{h}_{\ell}\right\} .
$$

Proof. Clearly $e=\left\{1 \leq j \leq m \mid \operatorname{Ad}(s) X_{j} \notin \mathfrak{g}_{j-1}+\mathfrak{h}_{l}\right\}$, where $l=s g$. But since $\operatorname{Ad}(s) X_{j}=X_{j}\left(\bmod \mathfrak{g}_{j-1}\right)$ the lemma is proved.

Using the notation from $[9, \S 1.2]$, we get from Lemma 2.2.2 that $O_{e}=O$.

Lemma 2.2.3. Let $l \in O$. Any element $X \in \mathfrak{g}$ can be written uniquely

$$
X=\sum_{u=1}^{d / 2} a_{X, u}(l) X_{k_{u}}+X(l),
$$

where $X(l) \in \mathfrak{h}_{l}$ and $a_{X, u}(l) \in \mathbf{R}, u=1, \ldots, d / 2$. The functions $l \rightarrow a_{X, u}(l)$ : $O \rightarrow \mathbf{R}$ and $l \rightarrow X(l): O \rightarrow \mathfrak{g}$ are polynomial functions. Moreover, $a_{X, u} \in$ $\mathscr{P}_{F}^{0}(O)$ for all $X \in \mathfrak{g}, u=1, \ldots, d / 2$, and $X(h l)=X(l)$ for all $l \in O$, $h \in H_{l}$.

Proof. This is the analogue of Lemma 1.2.3 in [9]. All we have to prove is that $a_{X, u}$ are polynomial functions. To this end we pick $g \in O$, and select a basis $Y_{1}, \ldots, Y_{d / 2}$ of $\mathfrak{h}\left(\bmod \mathfrak{g}_{g}\right)$. We then have with $l=s g: B_{\ell}\left(\operatorname{Ad}(s) Y_{u}, X\right)=$ $\sum_{v=1}^{d / 2} B_{l}\left(\operatorname{Ad}(s) Y_{u}, X_{k_{v}}\right) a_{X, v}(l)$. Defining $\mathbf{B}(s)$ to be the $d / 2 \times d / 2$-matrix

$$
\begin{aligned}
\mathbf{B}(s) & =\left[B_{l}\left(\operatorname{Ad}(s) Y_{u}, X_{k_{v}}\right)\right]_{1 \leq u, v \leq d / 2} \\
& =\left[B_{g}\left(Y_{u}, \operatorname{Ad}\left(s^{-1}\right) X_{k_{v}}\right)\right]_{1 \leq u, v \leq d / 2},
\end{aligned}
$$

$\mathbf{C}(s)$ to be the $d / 2 \times 1$-matrix

$$
\begin{aligned}
\mathbf{C}(s) & =\left[B_{l}\left(\operatorname{Ad}(s) Y_{u}, X\right)\right]_{1 \leq u \leq d / 2} \\
& =\left[B_{g}\left(Y_{u}, \operatorname{Ad}\left(s^{-1}\right) X\right)\right]_{1 \leq u \leq d / 2},
\end{aligned}
$$


and $\mathbf{A}(l)$ to be the $d / 2 \times 1$-matrix

$$
\mathbf{A}(l)=\left[a_{X, v}(l)\right]_{1 \leq v \leq d / 2}
$$

we have that $\mathbf{B}(s)$ is regular, and $\mathbf{C}(s)=\mathbf{B}(s) \mathbf{A}(l)$, hence $\mathbf{A}(l)=\mathbf{B}(s)^{-1} \mathbf{C}(s)$. Now clearly the matrix functions $s \rightarrow \mathbf{A}(s)$ and $s \rightarrow \mathbf{B}(s)$ have polynomial entries. It therefore suffices to prove that the function $s \rightarrow \operatorname{det} \mathbf{B}(s)$ is constant on $G$. To this end we note that we can write $\operatorname{Ad}\left(s^{-1}\right) X_{k_{v}}=\sum_{w=1}^{d / 2} t_{w v} X_{k_{w}}+W_{v}$, where $W_{v} \in \mathfrak{h}$, and where $t_{v v}=1$ while $t_{w v}=0$ for $w>v$. This means that $\mathbf{B}(s)=\mathbf{B}\left(e_{G}\right) \mathbf{T}$, where $e_{G}$ is the neutral element in $G$, and where $\mathbf{T}$ is the matrix $\left[t_{w v}\right]_{1 \leq w, v \leq d / 2}$, and clearly $\operatorname{det} \mathbf{T}=1$. But this shows that $\operatorname{det} \mathbf{B}(s)=$ $\operatorname{det} \mathbf{B}\left(e_{G}\right)$, and this ends the proof of the lemma.

Recall that $\pi: O \rightarrow G / H$ is the map $\pi: l=s g \rightarrow s H$. The map $\pi$ is a polynomial map (cf. Lemma 2.2.1).

Lemma 2.2.4. For any $X \in \mathfrak{g}$ we can write $\psi^{X}$ uniquely as

$$
\psi^{X}=\sum_{u=1}^{d / 2} a_{X, u} \psi^{X_{k_{u}}}+a_{X, 0},
$$

where $a_{X, 0}, a_{X, 1}, \ldots, a_{X, d / 2} \in \mathscr{P}_{F}^{0}(O)$.

Proof. The uniqueness follows from [9, Lemma 1.2.3]. To prove the existence we use Lemma 2.2.3 to write

$$
X=\sum_{u=1}^{d / 2} a_{X, u}(l) X_{k_{u}}+X(l)
$$

for each $l \in O$, where $X(l) \in \mathfrak{h}_{\ell}$. Setting $a_{X, 0}(l)=\langle l, X(l)\rangle$ we have

$$
\psi^{X}=\sum_{u=1}^{d / 2} a_{X, u} \psi^{X_{k_{u}}}+a_{X, 0} .
$$

Since already $a_{X, 1}, \ldots, a_{X, d / 2} \in \mathscr{P}_{F}^{0}(O)$, we only need to prove that $a_{X, 0} \in$ $\mathscr{P}_{F}^{0}(O)$. But clearly $a_{X, 0}$ is a polynomial function, since $l \rightarrow X(l)$ is a polynomial map. For $h \in H_{l}$ we have $a_{X, 0}(h l)=\langle h l, X(h l)\rangle=\langle h l, X(l)\rangle$ $=\left\langle l, \operatorname{Ad}\left(h^{-1}\right) X(l)\right\rangle=\langle l, X(l)\rangle=a_{X, 0}(l)$, since $\mathfrak{h}_{l}$ is a polarization at $l$. This erids the proof of the lemma.

Lemma 2.2.5. For each $X \in \mathfrak{g}$ we can write $\psi^{X}$ uniquely as

$$
\psi^{X}=\sum_{u=1}^{d / 2} b_{X, u} \circ \pi \psi^{X_{k_{u}}}+b_{X, 0} \circ \pi,
$$

where $b_{X, 0}, b_{X, 1}, \ldots, b_{X, d / 2} \in \mathscr{P}(G / H)$. 
Proof. We have from Lemma 2.2.1 that the map $\varphi \rightarrow \varphi \circ \pi: \mathscr{P}(G / H) \rightarrow \mathscr{P}_{F}^{0}(O)$ is a bijection, and the result therefore follows from the previous lemma.

Lemma 2.2.6. The map $\Phi: O \rightarrow \mathbf{R}^{d / 2} \times G / H$ given by

$$
\Phi: l \rightarrow\left(\left\langle l, X_{k_{i}}\right\rangle, \ldots,\left\langle l, X_{k_{d / 2}}\right\rangle, \pi(l)\right)
$$

is a bi-polynomial diffeomorphism.

Proof. It follows from [9, Lemma 1.2.4] that $\Phi$ is a diffeomorphism onto $\mathbf{R}^{d / 2} \times G / H$, and clearly $\Phi$ is a polynomial map. We just have to prove that the inverse of $\Phi$ is polynomial. To do this, let $\tau: G \rightarrow \mathbf{R}^{d / 2}$ be a polynomial chart on $G / H$, and define the polynomial chart $\tilde{\tau}: \mathbf{R}^{d / 2} \times G / H \rightarrow \mathbf{R}^{d / 2} \times \mathbf{R}^{d / 2}=\mathbf{R}^{d}$ by $\tilde{\tau}=\mathrm{id} \times \tau$. We have to prove that $\Phi^{-1} \circ \tilde{\tau}^{-1}: \mathbf{R}^{d} \rightarrow O$ is a polynomial map, and for this it suffices to prove that the map $(v, w) \rightarrow\left\langle X, \Phi^{-1} \circ \tilde{\tau}^{-1}(v, w)\right\rangle$ : $\mathbf{R}^{d} \rightarrow \mathbf{R}$ is polynomial for each $X \in \mathfrak{g}$. For $(v, w) \in \mathbf{R}^{d / 2} \times \mathbf{R}^{d / 2}$, set $l=\Phi^{-1} \circ \tilde{\tau}^{-1}(v, w)$. Then $\left(\left\langle l, X_{k_{1}}\right\rangle, \ldots,\left\langle l, X_{k_{d / 2}}\right\rangle, \pi(l)\right)=\Phi(l)=\tilde{\tau}^{-1}(v, w)=$ $\left(v, \tau^{-1}(w)\right)$. It follows that $v_{u}=\left\langle l, X_{k_{u}}\right\rangle$ for $1 \leq u \leq d / 2$, and that $\pi(l)=$ $\tau^{-1}(w)$. With the notation from Lemma 2.2 .5 we then get that

$$
\left\langle X, \Phi^{-1} \circ \tilde{\tau}^{-1}(v, w)\right\rangle=\langle l, X\rangle=\sum_{u=1}^{d / 2} b_{X, u}\left(\tau^{-1}(w)\right) v_{u}+b_{X, 0}\left(\tau^{-1}(w)\right) .
$$

Since the functions $b_{X, u}, u=0,1, \ldots, d / 2$, are polynomial we have proved the lemma.

Let $\tau$ be a polynomial chart on $G / H$ and write $\tau=\left(x_{1}, \ldots, x_{d / 2}\right)$. Define now the functions $y_{1}, \ldots, y_{d}$ on $O$ by $y_{u}(l)=\left\langle l, X_{k_{u}}\right\rangle$ and $y_{d / 2+u}(l)=$ $x_{u}(\pi(l))$, and define the map $\sigma: O \rightarrow \mathbf{R}^{d}$ by $\sigma=\left(y_{1}, \ldots, y_{d}\right)$.

Lemma 2.2.7. $\sigma$ defines a polynomial chart on $O$.

Proof. Since $\sigma(l)=\left(\left\langle l, X_{K_{1}}\right\rangle, \ldots,\left\langle l, X_{k_{d / 2}}\right\rangle, x_{1}(\pi(l)), \ldots, x_{d / 2}(\pi(l))\right)$, this is immediate from the previous lemma.

Lemma 2.2.8. We have

$$
\begin{aligned}
& \mathscr{P}_{F}^{0}(O)=\left\{\varphi \in \mathscr{P}(O) \mid \partial \varphi / \partial y_{u}=0,1 \leq u \leq d / 2\right\} \\
& \mathscr{P}_{F}^{1}(O)=\left\{\varphi \in \mathscr{P}(O) \mid \partial^{2} \varphi / \partial y_{u} \partial y_{v}=0,1 \leq u, v \leq d / 2\right\} .
\end{aligned}
$$

Proof. This follows from Lemma 2.2.7 and [9, Lemma 1.2.8].

Proposition 2.2.9. The space $\mathscr{P}_{F}^{1}(O)$ is a free $\mathscr{P}_{F}^{0}(O)$-module with basis $1, \psi^{X_{k_{1}}}$, $\ldots, \psi^{X_{k_{d / 2}}}$.

Proof. This follows immediately from Lemma 2.2.8, cf. [9, Lemma 1.2.9]. This ends the proof of the lemma. 
Lemma 2.2.10. For each $l \in O$ we have

$$
F_{l}=\left\{\left(\xi_{\varphi}\right)_{l} \mid \varphi \in \mathscr{P}_{F}^{0}(O)\right\}
$$

Proof. If $\varphi \in \mathscr{P}_{F}^{0}(O)$ we have that $\left(\xi_{\varphi}\right)_{l} \in F_{l}$ by the definition of $\mathscr{P}_{F}^{0}(O)$. Now it follows from $\left[9\right.$, Lemma 1.2.6] that the tangent vectors $\left(\xi_{y_{d / 2+1}}\right)_{l}, \ldots,\left(\xi_{y_{d}}\right)_{l}$ span $F_{l}$, and since $y_{u}$ belongs to $\mathscr{P}_{F}^{0}(O)$ for $d / 2<u \leq d$ we have proved the lemma.

Proposition 2.2.11. We have

$$
\begin{aligned}
& \mathscr{V}_{F}^{0}(O)=\left\{\xi \in \mathscr{V}(O) \mid \xi\left(\mathscr{P}_{F}^{0}(O)\right)=0\right\} \\
& \mathscr{V}_{F}^{1}(O)=\left\{\xi \in \mathscr{V}(O) \mid \xi\left(\mathscr{P}_{F}^{0}(O)\right) \subset \mathscr{E}_{F}^{0}(O)\right\} .
\end{aligned}
$$

Proof. This is proved just like Proposition 1.2.16 in [9], using Lemma 2.2.10.

Proposition 2.2.12. We have

$$
\begin{aligned}
& \mathscr{E}_{F}^{0}(O)=\left\{\varphi \in \mathscr{E}(O) \mid\left\{\varphi, \mathscr{P}_{F}^{0}(O)\right\}=0\right\}, \\
& \mathscr{E}_{F}^{1}(O)=\left\{\varphi \in \mathscr{E}(O) \mid\left\{\varphi, \mathscr{P}_{F}^{0}(O)\right\} \subset \mathscr{E}_{F}^{0}(O)\right\} .
\end{aligned}
$$

Proof. This follows immediately from Proposition 2.2.11.

Proposition 2.2.13. The space $\mathscr{P}_{F}^{0}(O)$ is a maximal commutative subalgebra of $\mathscr{P}(O)$, and $\mathscr{P}_{F}^{1}(O)$ is the normalizer of $\mathscr{P}_{F}^{0}(O)$ in $\mathscr{P}(O)$.

Proof. This follows immediately from Proposition 2.2.12.

2.3. Polynomial quantized operators. Let now $\chi: H \rightarrow \mathbf{T}$ be the continuous homomorphism with $\chi(\exp X)=e^{i\langle g, X\rangle}$ for $X \in \mathfrak{h}$. We know that the space $\mathscr{B}^{1}(G, \chi)$ in a natural way is an $\mathscr{E}_{F}^{0}(O)$-module. It follows from Lemma 2.2.1 that similarly we can consider $\mathscr{A}^{1}(G, \chi)$ as a $\mathscr{P}_{F}^{0}(O)$-module. With this terminology we get from Proposition 1.7.4:

Proposition 2.3.1. The space $\mathscr{A}^{1}(G, \chi)$ is a free $\mathscr{P}_{F}^{0}(O)$-module with basis 1 , $d \gamma_{\chi}\left(X_{k_{1}}\right), \ldots, d \gamma_{\chi}\left(X_{k_{d / 2}}\right)$.

2.4. Quantization and nilpotent groups. We now consider the quantization map $\delta_{\chi}: \mathscr{E}_{F}^{1}(O) \rightarrow \mathscr{B}^{1}(G, \chi)$. As shown in [9], $\delta_{\chi}$ is a Lie algebra isomorphism. It follows from Proposition 1.7.4 and Proposition 2.2.9 that the map $\varphi \rightarrow \delta_{\chi}(\varphi)$ carries $\mathscr{P}_{F}^{1}(O)$ into $\mathscr{A}^{1}(G, \chi)$, and, in fact, also onto $\mathscr{A}^{1}(G, \chi)$. We thus have Theorem 2.4.1. The map

$$
\delta_{\chi}: \mathscr{P}_{F}^{1}(O) \rightarrow \mathscr{A}^{1}(G, \chi)
$$

is a (Lie-algebra) isomorphism.

2.5. Unitary representations. We now consider the connection with unitary representations. Let $\mu$ be an invariant measure on $G / H$ and let $\pi_{\chi}$ be the representation $\operatorname{ind}_{H \uparrow G} \chi$ of $G$ on $L_{\mu}^{2}(G, \chi)$. The representation $\pi_{\chi}$ is in 
the equivalence class of irreducible representations associated with the orbit $O$. Associated with $\mu$ there is defined an involution $\sharp$ on $\mathscr{E}_{F}^{1}(O)$ such that $\delta_{\chi}\left(\varphi^{\sharp}\right)=\delta_{\chi}(\varphi)^{*}$, where $\delta_{\chi}(\varphi)^{*}$ is the formal adjoint of $\delta_{\chi}(\varphi)$ considered as an operator on $\mathscr{D}(G, \chi) \subset L_{\mu}^{2}(G, \chi)[9, \S 3]$.

Lemma 2.5.1. $\mathscr{P}_{F}^{1}(O)$ is invariant under the involution ${ }^{\sharp}$.

Proof. Since $\mu$ is invariant it follows that the function $w_{X}$ from $[9, \S 3.1]$ is equal to 0 . Therefore we get from [9, Lemma 3.1.7] that $\lambda\left(\varphi \psi^{X}\right)=\left\{\psi^{X}, \varphi\right\} \in$ $\mathscr{P}_{F}^{0}(O)$ for $\varphi \in \mathscr{P}_{F}^{0}(O), X \in \mathfrak{g}$. Since $\lambda(\varphi)=0$ for $\varphi \in \mathscr{P}_{F}^{0}(O)$ we get from Proposition 2.2.9 that if $\varphi \in \mathscr{P}_{F}^{1}(O)$, then $\lambda(\varphi) \in \mathscr{P}_{F}^{0}(O)$. This proves the lemma, since $\varphi^{\sharp}=-\bar{\varphi}+i \lambda(\bar{\varphi})$ for $\varphi \in \mathscr{E}_{F}^{1}(O)[9, \S 3.1]$.

In $[9, \S 3.1]$ we have also defined a map $\varepsilon_{\chi}: \mathscr{E}_{F}^{1}(O) \rightarrow \mathscr{B}^{1}(G, \chi)$. Using the previous lemma we get from [9, Theorem 3.1.13]:

Theorem 2.5.2. The map $\varepsilon_{\chi}: \mathscr{P}_{F}^{1}(O) \rightarrow \mathscr{A}^{1}(G, \chi)$ is a Lie algebra isomorphism, and

$$
\varepsilon_{\chi}(\varphi)^{*}=-\varepsilon_{\chi}(\bar{\varphi})
$$

for all $\varphi \in \mathscr{P}_{F}^{1}(O)$, and

$$
\varepsilon_{\chi}\left(\psi^{X}\right) f=d \pi(X) f
$$

for all $f \in \mathscr{D}(G, \chi), X \in \mathfrak{g}$.

2.6. Global canonical coordinates. We can now prove

Theorem 2.6.1. There exists functions $q_{1}, \ldots, q_{d / 2} \in \mathscr{P}_{F}^{0}(O)$, and functions $p_{1}, \ldots, p_{d / 2} \in \mathscr{P}_{F}^{1}(O)$ such that $\left(p_{1}, \ldots, p_{d / 2}, q_{1}, \ldots, q_{d / 2}\right)$ is a set of canonical coordinates on $O$. Moreover, any such choice of canonical coordinates defines a polynomial chart on $O$.

Proof. Since $\mathscr{A}^{1}(G, \chi)$ and $\mathscr{A}^{1}\left(\mathbf{R}^{d / 2}\right)$ are isomorphic as Lie algebras, there exist skewadjoint elements $D_{1}, \ldots D_{d / 2}$ in $\mathscr{A}^{1}(G, \chi)$ and selfadjoint elements $M_{1}, \ldots, M_{d / 2}$ in $\mathscr{A}^{0}(G, \chi)$ such that $\left[D_{u}, D_{v}\right]=0,\left[M_{u}, M_{v}\right]=0$ and $\left[D_{u}, M_{v}\right]=i \delta_{u v}, 1 \leq u, v \leq d / 2$. From Theorem 2.5.2 we get that there exist real functions $p_{1}, \ldots, p_{d / 2}$ in $\mathscr{P}_{F}^{1}(O)$ such that $\varepsilon_{\chi}\left(p_{u}\right)=D_{u}$ and real functions $q_{1}, \ldots, q_{d / 2}$ in $\mathscr{P}_{F}^{0}(O)$ such that $\varepsilon_{\chi}\left(q_{u}\right)=i M_{u}, 1 \leq u \leq d / 2$. We can then show that the set $\left(p_{1}, \ldots, p_{d / 2}, q_{1}, \ldots, q_{d / 2}\right)$ defines canonical coordinates just like in [9, proof of Theorem 2.2.1].

Let us then show that any such choice $\sigma=\left(p_{1}, \ldots, p_{d / 2}, q_{1}, \ldots, q_{d / 2}\right)$ of canonical coordinates defines a polynomial chart. Set $U=\sigma(O)$ ( $U$ will be shown to be equal to $\mathbf{R}^{d}$ in the following). We have to prove that the map $\sigma^{-1}: U \rightarrow O$ is a polynomial map. To do this, it suffices to show that the map 
$(v, w) \rightarrow\left\langle X, \sigma^{-1}(v, w)\right\rangle: U \rightarrow \mathbf{R}$ is a polynomial map. We write

$$
\psi^{X}=\sum_{u=1}^{d / 2} a_{X, u}(q) p_{u}+a_{X, 0}(q)
$$

Here $a_{X, 0}, a_{X, 1}, \ldots, a_{X, d / 2}$ are polynomial functions, since $a_{X, u}=\left\{\psi^{X}, q_{u}\right\}$ (Lemma 2.1.1). Now if $l=\sigma^{-1}(v, w)$, then $p_{u}(l)=v_{u}$ and $q_{u}(l)=w_{u}$, and it follows that

$$
\left\langle X, \sigma^{-1}(v, w)\right\rangle=\langle X, l\rangle=\sum_{u=1}^{d / 2} a_{X, u}(v) w_{u}+a_{X, 0}(v)
$$

This shows that the map $(v, w) \rightarrow\left\langle X, \sigma^{-1}(v, w)\right\rangle$ is a polynomial function, and this ends the proof of the theorem.

Remark 2.6.2. Although it is known that there exists global canonical coordinates on $O$ ([1], cf. [9]) the result in the previous theorem is considerably stronger. What we have shown here is that for any choice of a real polarization there exists polynomial global canonical coordinates compatible with this polarization.

\subsection{Construction of unitary representations using global canonical coordinates.}

Let $p_{1}, \ldots, p_{d / 2}, q_{1}, \ldots, q_{d / 2}$ be global canonical coordinates on $O$ such that $q_{1}, \ldots, q_{d / 2} \in \mathscr{P}_{F}^{0}(O)$, and $p_{1}, \ldots, p_{d / 2} \in \mathscr{P}_{F}^{1}(O)$. Such coordinates exists by Theorem 2.6.1, and, moreover, they define a polynomial chart on $O$.

We define the function $\tau: G / H \rightarrow \mathbf{R}^{d / 2}$ by $\tau(s H)=\left(q_{1}(s g), \ldots, q_{d / 2}(s g)\right)$. Clearly $\tau$ is well defined since $q_{1}, \ldots, q_{d / 2}$ belong to $\mathscr{P}_{F}^{0}(O)$, and $\tau$ is a global chart [9, Lemma 3.2.1] whose image is $\mathbf{R}^{d / 2}$. It is easily seen that the quasiinvariant measure $\mu$ defined by $\tau[9, \S 3.2]$ is in fact invariant.

Lemma 2.7.1. The polynomial quantizable functions $\mathscr{P}_{F}^{1}(O)$ are the functions $\varphi$ of the form

$$
\varphi=\sum_{u=1}^{d / 2} a_{u}(q) p_{u}+a_{0}(q)
$$

where $a_{0}, a_{1}, \ldots, a_{d / 2} \in \mathscr{P}\left(\mathbf{R}^{d / 2}\right)$.

Proof. This is clear, since $\left(p_{1}, \ldots, p_{d / 2}, q_{1}, \ldots, q_{d / 2}\right)$ is a polynomial chart.

It follows from Lemma 2.7.1 that we in particular can write

$$
\psi^{X}=\sum_{u=1}^{d / 2} a_{X, u}(q) p_{u}+a_{X, 0}(q)
$$

for each $X \in \mathfrak{g}$, where $a_{X, u} \in \mathscr{P}\left(\mathbf{R}^{d / 2}\right), u=0,1, \ldots, d / 2$. 
From [9, Theorem 3.2.3] we can then derive

Theorem 2.7.2. There exists one and only one strongly continuous, unitary representation $\pi$ of $G$ on $L^{2}\left(\mathbf{R}^{d / 2}\right)$ such that $\mathscr{D}\left(\mathbf{R}^{d / 2}\right) \subset H_{\pi}^{\infty}$, and such that

$$
d \pi(X) f(t)=\sum_{u=1}^{d / 2} a_{X, u}(t) \frac{\partial f}{\partial t_{u}}(t)+i a_{X, 0}(t) f(t)+\frac{1}{2} \sum_{u=1}^{d / 2} \frac{\partial a_{X, u}}{\partial t_{u}}(t) f(t)
$$

for $f \in \mathscr{D}\left(\mathbf{R}^{d / 2}\right), X \in \mathfrak{g}$. The representation $\pi$ is equivalent to $\pi_{\chi}$, i.e., it is in the equivalence class of irreducible representations associated with the orbit $O$.

\section{QUANTIZATION AND THE UNIVERSAL ENVELOPING ALGEBRA}

In the following we shall consider the set of elements in the universal enveloping algebra which are mapped into the set of quantized operators by a given representation.

3.1. The map $\tilde{\Phi}$. Let $G$ be a connected and simply connected nilpotent Lie group with Lie algbra $\mathfrak{g}$. Let $g \in \mathfrak{g}^{*}$, and let $\mathfrak{h}$ be a real polarization at $g$. Let $\chi: H=\exp \mathfrak{h} \rightarrow \mathbf{T}$ be the unitary character with $\chi(\exp X)=e^{i\langle g, X\rangle}, X \in \mathfrak{h}$, and set $\pi_{\chi}=$ ind $_{H \uparrow G} \chi$. Then $\pi_{\chi}$ is the irreducible representation associated with the orbit $O=G g$. We have with the notation from $\S 1.7$ :

Theorem 3.1.1 (Kirillov). The differentiable vectors for $\pi_{\chi}$ are precisely $\mathscr{S}(G, \chi)$, and the image of $U\left(\mathfrak{g}_{\mathrm{C}}\right)$ by $d \pi_{\chi}$ is $\mathscr{A}(G, \chi)$.

This result is due to Kirillov [5]. More specifically he showed that there exists for each $g \in \mathfrak{g}^{*}$ a polarization $\mathfrak{h}$ at $g$ such that the result holds. It was observed in [2] that it is actually true for any real polarization $\mathfrak{h}$ at $g$.

We set

$$
I(O)=\operatorname{ker}\left(d \pi_{\chi}\right)
$$

which is a selfadjoint primitive ideal in $U\left(\mathfrak{g}_{\mathrm{C}}\right)([4])$. The ideal $I(O)$ is equal to the annahilator of the left $U\left(\mathfrak{g}_{\mathbf{C}}\right)$-module $U\left(\mathfrak{g}_{\mathbf{C}}\right) \otimes_{U\left(\mathfrak{h}_{\mathbf{c}}\right)} \mathbf{C}$ [4]. In [7] I have given an explicit formula for $I(O)$. In the following we shall develop further the ideas put forward in [7]. Let $F$ be the $G$-invariant polarization of $O$ defined by $\mathfrak{h}$, cf. [9], and set

$$
J_{F}^{0}(O)=\left\{u \in U\left(\mathfrak{g}_{\mathbf{C}}\right) \mid d \pi_{\chi}(u) \in \mathscr{A}^{0}(G, \chi)\right\}
$$

and

$$
J_{F}^{1}(O)=\left\{u \in U\left(\mathfrak{g}_{\mathbf{C}}\right) \mid d \pi_{\chi}(u) \in \mathscr{A}^{1}(G, \chi)\right\} .
$$

(It is easily seen that the definition of $J_{F}^{0}(O)$ and $J_{F}^{1}(O)$ depends only on $F$.) In other words, $J_{F}^{0}(O)\left(J_{F}^{1}(O)\right)$ is the set of elements in $U\left(\mathfrak{g}_{\mathrm{C}}\right)$ which are mapped by $d \pi_{\chi}$ to multiplication operators (differential operators of order at most one). Obviously we have

$$
I(O) \subset J_{F}^{0}(O) \subset J_{F}^{1}(O),
$$


and by Kirillov's theorem we have

$$
d \pi_{\chi}\left(J_{F}^{0}(O)\right)=\mathscr{A}^{0}(G, \chi)
$$

and

$$
d \pi_{\chi}\left(J_{F}^{1}(O)\right)=\mathscr{A}^{1}(G, \chi) .
$$

Factoring $d \pi_{\chi}$ through $I(O)$ we get a Lie algebra isomorphism $d \tilde{\pi}_{\chi}$ :

$$
J_{F}^{1}(O) / I(O) \stackrel{d \tilde{\pi}_{\chi}}{\longrightarrow} \mathscr{A}^{1}(G, \chi)
$$

such that

$$
J_{F}^{0}(O) / I(O) \stackrel{d \tilde{\pi}_{\chi}}{\longrightarrow} \mathscr{A}^{0}(G, \chi) .
$$

Now the quantization map

$$
\delta_{\chi}: \mathscr{P}_{F}^{1}(O) \rightarrow \mathscr{A}^{1}(G, \chi)
$$

is also a Lie algebra isomorphism (Theorem 2.4.1). This means that there is a Lie algebra isomorphism $\tilde{\Phi}: \mathscr{P}_{F}^{1}(O) \rightarrow J_{F}^{1}(O) / I(O)$ which makes the following diagram commutative:

$$
\begin{array}{ccc}
\mathscr{P}_{F}^{1}(O) & \stackrel{\tilde{\Phi}}{\longrightarrow} & J_{F}^{1}(O) / I(O) \\
\delta_{\chi} \searrow & \swarrow d \tilde{\pi}_{\chi}
\end{array} \underset{\mathscr{A}^{1}(G, \chi)}{ }
$$

The goal of the following is to give an explicit description of $\tilde{\boldsymbol{\Phi}}$ in the case where $\mathfrak{h}$ is a Vergne polarization. Specifically, we shall construct for a given Jordan-Hölder basis $\mathscr{B}: X_{1}, \ldots, X_{m}$ and associated Vergne polarization $\mathfrak{h}$ an explicit map $\Phi=\Phi_{O}^{\mathscr{B}}: \mathscr{P}_{F}^{1}(O) \rightarrow J_{F}^{1}(O), F$ being the polarization of $O$ associated with $\mathfrak{h}$, such that $\Phi$ composed with the quotient map $J_{F}^{1}(O) \rightarrow$ $J_{F}^{1}(O) / I(O)$ is the map $\tilde{\Phi}$.

Let $\mathscr{B}: X_{1}, \ldots, X_{m}$ be a Jordan-Hölder basis in $\mathfrak{g}$, and set

$$
\mathfrak{g}_{j}=\operatorname{span}\left(X_{1}, \ldots, X_{j}\right), \quad 1 \leq j \leq m \text {. }
$$

Then

$$
\mathscr{F}: \mathfrak{g}=\mathfrak{g}_{m} \supset \mathfrak{g}_{m-1} \supset \cdots \supset \mathfrak{g}_{1} \supset \mathfrak{g}_{0}=\{0\}
$$

is a Jordan-Hölder sequence in $\mathfrak{g}$. Let $g \in \mathfrak{g}^{*}$ and set $g_{j}=g \mid \mathfrak{g}_{j}$ for each $1 \leq j \leq m$. The linear subspace

$$
\mathfrak{h}(g, \mathscr{F})=\sum_{j=1}^{m}\left(\mathfrak{g}_{j}\right)_{g_{j}}
$$

is then a polarization at $g \in \mathfrak{g}^{*}$, the so-called Vergne polarization at $g \in \mathfrak{g}^{*}$ associated with $\mathscr{F}$ [3]. We shall call the associated polarization of $O$ for $F(O, \mathscr{F})$, cf. [9]. We set $H(g, \mathscr{F})=\exp \mathfrak{h}(g, \mathscr{F})$.

Let $\sigma=\sigma_{O}^{\mathscr{B}}: O \rightarrow \mathbf{R}^{d}$ be the $\mathscr{B}$-chart of $O$, see $\S 1.6$. For each $\varphi \in$ $\mathscr{P}(O)$ the function $\varphi \circ \sigma^{-1}$ is a polynomial in $\mathbf{C}\left[y_{1}, \ldots, y_{d}\right]$. The element 
$\varphi \circ \sigma^{-1}\left(-i X_{j_{1}}, \ldots,-i X_{j_{d}}\right)$ is then well defined in $S\left(\mathfrak{g}_{\mathbf{C}}\right)$, the symmetric algebra of $\mathfrak{g}_{\mathrm{C}}$. For $\varphi \in \mathscr{P}_{F(O, \mathscr{F})}^{0}(O)$ we set

$$
\Phi(\varphi)=\Phi_{O}^{\mathscr{B}}(\varphi)=i \omega\left(\varphi \circ \sigma^{-1}\left(-i X_{j_{1}}, \ldots,-i X_{j_{d}}\right)\right),
$$

where $\omega: S\left(\mathfrak{g}_{\mathrm{C}}\right) \rightarrow U\left(\mathfrak{g}_{\mathrm{C}}\right)$ is the symmetrization map. If $d=\operatorname{dim} O=0$ we set $\Phi(\varphi)=i \varphi(O) \mathbf{1}$, where 1 is the unit in $U\left(\mathfrak{g}_{\mathrm{C}}\right)$.

Theorem 3.1.2. For $\varphi \in \mathscr{P}_{F(O, \mathscr{F})}^{0}(O)$ we have $d \pi_{\chi}(\Phi(\varphi))=\delta_{\chi}(\varphi)$.

It follows from the theorem that the value of $\tilde{\Phi}$ on $\varphi \in \mathscr{P}_{F(O, \mathscr{F})}^{0}(O)$ is the canonical image of $\Phi(\varphi)$ in $J_{F}^{0}(O) / I(O)$.

Proof of theorem. The proof is by induction on the dimension of $\mathfrak{g}$. If $\operatorname{dim} \mathfrak{g}=$ 1 the result is true by definition. Suppose then that the result has been proved for all dimensions less than $m$, and that $\operatorname{dim} \mathfrak{g}=m$. Let $\mathfrak{z}$ be the center of $\mathfrak{g}$, and set $\mathfrak{z}_{0}=\operatorname{ker} g \mid \mathfrak{z}$. Suppose first that we are in

Case (a): $\operatorname{dim}_{\mathfrak{z}_{0}}>0$. Set $\tilde{\mathfrak{g}}=\mathfrak{g} / \mathfrak{z}_{0}$, and let $c: \mathfrak{g} \rightarrow \tilde{\mathfrak{g}}$ be the quotient map. We let also $c$ denote the quotient map $c: G \rightarrow \tilde{G}=G / Z_{0}$, where $Z_{0}=\exp _{\mathfrak{z}_{0}}$. Let $\tilde{g}$ be the element in $\tilde{\mathfrak{g}}^{*}$ such that $\tilde{g} \circ c=g$.

We now define a Jordan-Hölder sequence in $\tilde{\mathfrak{g}}$. We set $I=\left\{1 \leq j \leq m \mid \mathfrak{g}_{j} \not \subset\right.$ $\left.\mathfrak{g}_{j-1}+\mathfrak{z}_{0}\right\}$, and write $I=\left\{i_{1}<\cdots<i_{n}\right\}$ and $\tilde{\mathfrak{g}}_{r}=\left(\mathfrak{g}_{i_{r}}+\mathfrak{z}_{0}\right) / \mathfrak{z}_{0}, r=1, \ldots, n$. Then

$$
\tilde{\mathscr{F}}: \tilde{\mathfrak{g}}=\tilde{\mathfrak{g}}_{n} \supset \tilde{\mathfrak{g}}_{n-1} \supset \cdots \supset \tilde{\mathfrak{g}}_{1} \supset \tilde{\mathfrak{g}}_{0}=\{0\}
$$

is a Jordan-Hölder sequence in $\tilde{\mathfrak{g}}$, and setting $\tilde{X}_{r}=c\left(X_{i_{r}}\right)$ we have that $\tilde{\mathscr{B}}: \tilde{X}_{1}, \ldots, \tilde{X}_{n}$ is a Jordan-Hölder basis in $\tilde{\mathfrak{g}}$ compatible with $\tilde{\mathscr{F}}$. We note that $\mathfrak{z}_{0} \subset \mathfrak{g}_{g}$, and that $\tilde{\mathfrak{g}}_{\dot{g}}=\mathfrak{g}_{g} / \mathfrak{z}_{0}$.

For $1 \leq j \leq m$ we define the subalgebra $\mathfrak{g}_{j}^{\sharp}$ of $\mathfrak{g}$ by $\mathfrak{g}_{j}^{\sharp}=\mathfrak{g}_{j}+\mathfrak{z}_{0}$, and we set $g_{j}^{\sharp}=g \mid \mathfrak{g}_{j}^{\sharp}$. It is then immediate that $\left(\mathfrak{g}_{j}^{\sharp}\right)_{g_{j}^{\sharp}}=\left(\mathfrak{g}_{j}\right)_{g_{j}}+\mathfrak{z}_{0}$. Suppose now that $i_{r} \leq j<i_{r+1}, 1 \leq r<n$. Then clearly $\mathfrak{g}_{i_{r}}^{\sharp} \subset \mathfrak{g}_{j}^{\sharp}$, but we also have that $\mathfrak{g}_{j} \subset \mathfrak{g}_{i_{r}}+\mathfrak{z}_{0}$, and therefore that $\mathfrak{g}_{j}+\mathfrak{z}_{0} \subset \mathfrak{g}_{i_{r}}+\mathfrak{z}_{0}$, so $\mathfrak{g}_{i_{r}}^{\sharp}=\mathfrak{g}_{j}^{\sharp}$. We next note that since $\mathfrak{z}_{0} \subset \mathfrak{g}_{g} \subset \mathfrak{h}(g, \mathscr{F})$ we have that $\mathfrak{h}(g, \mathscr{F})=\mathfrak{h}(g, \mathscr{F})+\mathfrak{z}_{0}=\sum_{j=1}^{m}\left(\mathfrak{g}_{j}\right)_{g_{j}}+$ $\mathfrak{z}_{0}=\sum_{j=1}^{m}\left(\left(\mathfrak{g}_{j}\right)_{g_{j}}+\mathfrak{z}_{0}\right)=\sum_{j=1}^{m}\left(\mathfrak{g}_{j}^{\sharp}\right)_{g_{j}^{\sharp}}=\sum_{r=1}^{n}\left(\mathfrak{g}_{i_{r}}^{\sharp}\right)_{\mathfrak{i}_{r}^{\sharp}}$. For each $1 \leq r \leq n$ we have that $c\left(\mathfrak{g}_{i_{r}}^{\sharp}\right)=\tilde{\mathfrak{g}}_{r}$, and $\tilde{g}_{r} \circ\left(c \mid \mathfrak{g}_{i_{r}}^{\sharp}\right)=g_{i_{r}}^{\sharp}$. Since $\mathfrak{z}_{0} \subset\left(\mathfrak{g}_{i_{r}}^{\sharp}\right)_{g_{i_{r}}^{\sharp}}$ we find that $\left(\mathfrak{g}_{i_{r}}^{\sharp}\right)_{g_{i_{r}}^{\sharp}} / \mathfrak{z}_{0}=\left(\tilde{\mathfrak{g}}_{r}\right)_{\tilde{g}_{r}}$. The conclusion is that $c(\mathfrak{h}(g, \mathscr{F}))=\sum_{r=1}^{n} c\left(\left(\mathfrak{g}_{i_{r}}^{\sharp}\right)_{g_{i_{r}}^{\sharp}}\right)=$ $\sum_{r=1}^{n}\left(\tilde{\mathfrak{g}}_{r}\right)_{\tilde{g}_{r}}=\mathfrak{h}(\tilde{g}, \tilde{F})$, and therefore that $\mathfrak{h}(\tilde{g}, \tilde{\mathscr{F}})=\mathfrak{h}(g, \mathscr{F}) / \mathfrak{z}_{0}$, and that $\tilde{H}=H / Z_{0}$, where $\tilde{H}=H(\tilde{g}, \tilde{F})$ and $H=H(g, \mathscr{F})$.

We now consider the orbits $O=G g$ and $\tilde{O}=\tilde{G} \tilde{g}$. The map $\tilde{O} \rightarrow O$ given by $\tilde{l} \rightarrow \tilde{l} \circ c=l$ is a bijection. Also, if $\varphi \in \mathscr{P}(O)$, then the function $\tilde{\varphi}$ on $\tilde{O}$ defined by $\tilde{\varphi}(\tilde{l})=\varphi(l \circ c)$ is in $\mathscr{P}(\tilde{O})$; in fact, for $X \in \mathfrak{g}$ we have $\left(\psi^{X}\right)^{\sim}=\psi^{\tilde{X}}$, where $\tilde{X}=c(X)$, and the assertion then follows from Lemma 1.7.2. It follows 
that the map $\varphi \rightarrow \tilde{\varphi}$ is a linear bijection from $\mathscr{P}(O)$ onto $\mathscr{P}(\tilde{O})$. For $s \in G$, $h \in H$, let $\tilde{s}=c(s), \tilde{h}=c(h)$. We have $\tilde{\varphi}(\tilde{s} \tilde{h} \tilde{g})=\varphi(\operatorname{sh} g)$, so $\varphi \rightarrow \tilde{\varphi}$ maps $\mathscr{P}_{F(O, \mathscr{F})}^{0}(O)$ bijectively onto $\mathscr{P}_{F(\tilde{O}, \tilde{F})}^{0}(\tilde{O})$.

Let $\tilde{\chi}: \tilde{G} \rightarrow \mathbf{T}$ be the unitary character with $\tilde{\chi}(\exp \tilde{X})=\mathrm{e}^{i\langle\tilde{g}, \tilde{X}\rangle}, \tilde{X} \in$ $\mathfrak{h}(\tilde{g}, \tilde{F})$. Then $\tilde{\chi} \circ c=\chi$.

There is defined a map $V: L^{2}(G, \chi) \rightarrow L^{2}(\tilde{G}, \tilde{\chi})$ by $f \rightarrow \tilde{f}$, where $\tilde{f}=$ $f \circ c$. The map $V$ is clearly unitary and $V \pi_{\chi}(s) V^{-1}=\pi_{\tilde{\chi}}(\tilde{s})$, where $\pi_{\chi}=$ ind $_{H \uparrow G} \chi, \pi_{\tilde{\chi}}=\operatorname{ind}_{\tilde{H} \uparrow \tilde{G}} \tilde{\chi}$, and $\tilde{s}=c(s)$ for $s \in G$. It is immediately seen that $V$ maps $\mathscr{S}(G, \chi)$ onto $\mathscr{S}(\tilde{G}, \tilde{\chi})$. Moreover, $\delta_{\tilde{\chi}}(\tilde{\varphi}) \tilde{f}(\tilde{s})=\tilde{\varphi}(\tilde{s} \tilde{g}) \tilde{f}(\tilde{s})=$ $\varphi(s g) f(s)=\delta_{\chi}(\varphi) f(s)$, so $V \delta_{\chi}(\varphi) f=\delta_{\tilde{\chi}}(\tilde{\varphi}) V f$, or $V \delta_{\chi}(\varphi) V^{-1}=\delta_{\tilde{\chi}}(\tilde{\varphi})$.

Now we have to prove that $d \pi_{\chi}\left(\Phi_{O}^{\mathscr{B}}(\varphi)\right)=\delta_{\chi}(\varphi)$, or, equivalently,

$$
d\left(\pi_{\tilde{\chi}} \circ c\right)\left(\Phi_{O}^{\mathscr{B}}(\varphi)\right)=\delta_{\tilde{\chi}}(\tilde{\varphi}) \quad \text { or } \quad d \pi_{\tilde{\chi}}\left(c\left(\Phi_{O}^{\mathscr{B}}(\varphi)\right)=\delta_{\tilde{\chi}}(\tilde{\varphi}) .\right.
$$

By the induction hypothesis we have $d \pi_{\tilde{\chi}}\left(\Phi_{\tilde{O}}^{\tilde{\mathscr{B}}}(\tilde{\varphi})\right)=\delta_{\tilde{\chi}}(\tilde{\varphi})$, so we will be done if we can show that $c\left(\Phi_{O}^{\mathscr{B}}(\varphi)\right)=\Phi_{\tilde{O}}^{\tilde{\mathscr{B}}}(\tilde{\varphi})$.

To show that $c\left(\Phi_{O}^{\mathscr{B}}(\varphi)\right)=\Phi_{\tilde{O}}^{\mathscr{B}}(\tilde{\varphi})$ we start by noting that $J_{g} \subset I$, since $j \notin I \Rightarrow X_{j} \in \mathfrak{g}_{j-1}+\mathfrak{z}_{0} \Rightarrow X_{j} \in \mathfrak{g}_{j-1}+\mathfrak{g}_{g} \Rightarrow j \notin J_{g}$. Writing $e=J_{g}=\left\{j_{1}<\right.$ $\left.\cdots<j_{d}\right\}$ and $\tilde{e}=J_{\tilde{g}}=\left\{\tilde{j}_{1}<\cdots<\tilde{j}_{d}\right\}$ we have that $i_{\tilde{j}_{u}}=j_{u}$ for $u=1, \ldots, d$.

Let $\sigma_{O}^{\mathscr{B}}$ be the $\mathscr{B}$-chart of $O$, and let $\sigma_{\tilde{O}}^{\mathscr{B}}$ be the $\tilde{\mathscr{B}}$-chart of $\tilde{O}$. We have $\sigma_{O}^{\mathscr{B}}(\tilde{l} \circ c)_{u}=\left\langle\tilde{l} \circ c, X_{j_{u}}\right\rangle=\left\langle\tilde{l} \circ c, X_{i_{j_{u}}}\right\rangle=\left\langle\tilde{l}, c\left(X_{i_{j_{u}}}\right)\right\rangle=\left\langle\tilde{l}, \tilde{X}_{\tilde{j}_{u}}\right\rangle=\sigma_{\tilde{O}}^{\mathscr{\mathscr { B }}}(\tilde{l})_{u}$, so $\sigma_{O}^{\mathscr{B}}(\tilde{l} \circ c)=\sigma_{\tilde{O}}^{\tilde{\mathscr{B}}}(\tilde{l})$. Therefore, if $y \in \mathbf{R}^{d}$, and $\tilde{l}=\sigma_{\tilde{O}}^{\mathscr{\mathscr { B }}^{-1}}(y)$, then $\sigma_{O}^{\mathscr{B}}(\tilde{l} \circ c)=y$. It follows that $\tilde{\varphi} \circ \sigma_{\tilde{O}}^{\tilde{\mathscr{B}}^{-1}}(y)=\tilde{\varphi}(\tilde{l})=\varphi(\tilde{l} \circ c)=\varphi \circ \sigma_{O}^{\mathscr{B}}-1(y)$, so $\tilde{\varphi} \circ \sigma_{\tilde{O}}^{\tilde{\mathscr{B}}^{-1}}=$ $\varphi \circ \sigma_{O}^{\mathscr{B}}{ }^{-1}$. Therefore

$$
\varphi \circ \sigma_{O}^{\mathscr{B}}-1\left(-i X_{j_{1}}, \ldots,-i X_{j_{d}}\right)=\tilde{\varphi} \circ{\sigma_{\tilde{O}}^{\tilde{B}^{-1}}}^{-1}\left(-i X_{i_{j_{1}}}, \ldots,-i X_{i_{j_{d}}}\right)
$$

which implies $c\left(\varphi \circ \sigma_{O}^{\mathscr{B}}-1\left(-i X_{j_{1}}, \ldots,-i X_{j_{d}}\right)\right)=\tilde{\varphi} \circ \sigma_{\tilde{O}}^{\check{\mathscr{O}}^{-1}}\left(-i \tilde{X}_{\tilde{j}_{1}}, \ldots,-i \tilde{X}_{\tilde{j}_{d}}\right)$, and taking symmetrization $\omega$ and using that $c$ commutes with $\omega$ we get $c\left(\Phi_{O}^{\mathscr{B}}(\varphi)\right)=\Phi_{\tilde{O}}^{\mathscr{B}}(\tilde{\varphi})$. This finishes case $(\mathrm{a})$.

Suppose then that we are in

Case (b): $\operatorname{dim} \mathfrak{z}_{0}=0$. Then $\operatorname{dim} \mathfrak{z}=1$ and $\langle g, \mathfrak{z}\rangle \neq 0$, so $\mathfrak{z}=\mathfrak{g}_{1}$, and $\left\langle g, X_{1}\right\rangle \neq 0$. In particular $\left[\mathfrak{g}, \mathfrak{g}_{2}\right]=\mathfrak{g}_{1}$, and therefore $\mathfrak{g}_{2} \not \subset \mathfrak{g}_{g}$, hence $2 \in J_{g}=$ $e$, and $j_{1}=2$. Note also that $\mathfrak{g}_{g} \subset \mathfrak{k}=\operatorname{ker} a d X_{2}$ (since otherwise $\mathfrak{g}_{g}+\mathfrak{k}=\mathfrak{g}$ and therefore $\left\langle g, \mathfrak{g}_{1}\right\rangle=\left\langle g,\left[\mathfrak{g}, X_{2}\right]\right\rangle=\left\langle\mathfrak{g} g, X_{2}\right\rangle=\left\langle\mathfrak{k} g, X_{2}\right\rangle=0$ which is a contradiction). We then claim that we can assume that $\mathfrak{g}_{m-1}=\mathfrak{k}=\operatorname{ker}$ ad $X_{2}$.

Proof of claim. Clearly $\mathfrak{k}=\operatorname{ker}$ ad $X_{2}$ is an ideal in $\mathfrak{g}$ of codimension 1 . Set $p=\min \left\{1 \leq j \leq m \mid X_{j} \notin \mathfrak{k}\right\}$. Then $p$ is well defined, $p \geq 3$ and $\mathfrak{g}=\mathfrak{k} \oplus \mathbf{R} X_{p}$. 
It is easily seen that $p \in J_{g}$ (in fact, if $p \notin J_{g}$, then $X_{p} \in \mathfrak{g}_{g}+\mathfrak{g}_{p-1} \subset \mathfrak{g}_{g}+\mathfrak{k} \subset \mathfrak{k}$ which is a contradiction). We then define a new basis $\hat{\mathscr{B}}: \hat{X}_{1}, \ldots, \hat{X}_{m}$ in $\mathfrak{g}$ in the following way: For $1 \leq j \leq p-1$ we set $\hat{X}_{j}=X_{j}$, for $p \leq j \leq m-1$ we set $\hat{X}_{j}=X_{j+1}+c_{j+1} X_{p}$, where the $c_{j+1} \in \mathbf{R}$ are selected such that $\hat{X}_{j} \in \mathfrak{k}$ (this is possible since $\mathbf{R} X_{p} \oplus \mathfrak{k}=\mathfrak{g}$ ), and finally we set $\hat{X}_{m}=X_{p}$. We then define the linear subspaces $\hat{\mathfrak{g}}_{j}, j=1, \ldots, m$, in $\mathfrak{g}$ by

$$
\hat{\mathfrak{g}}_{j}=\mathbf{R} \hat{X}_{1} \oplus \cdots \oplus \mathbf{R} \hat{X}_{j} .
$$

We have $\hat{\mathfrak{g}}_{j}=\mathfrak{g}_{j}$ for $1 \leq j \leq p-1$ and $\mathfrak{g}_{j+1}=\hat{\mathfrak{g}}_{j} \oplus \mathbf{R} X_{p}$ for $p-1 \leq j \leq$ $m-1$ implying that $\hat{\mathfrak{g}}_{j}=\mathfrak{g}_{j+1} \cap \mathfrak{k}$ for $p-1 \leq j \leq m-1$. This shows that $\hat{\mathscr{F}}: \hat{\mathfrak{g}}_{1}, \ldots, \hat{\mathfrak{g}}_{m}$ is a Jordan-Hölder sequence in $\mathfrak{g}$, and $\hat{\mathscr{B}}$ is compatible with $\hat{\mathscr{F}}$ by construction. By construction we also have $\hat{\mathfrak{g}}_{m-1}=\mathfrak{k}$. We designate the objects associated with this new Jordan-Hölder sequence $\hat{J}_{g}, \hat{e}, \mathfrak{h}(O, \hat{\mathscr{F}})$ etc. We write $\hat{J}_{g}=\hat{e}=\left\{\hat{\jmath}_{1}<\cdots<\hat{j}_{d}\right\}$.

For $1 \leq j \leq p-1$ we clearly have that $j \in J_{g} \Leftrightarrow j \in \hat{J}_{g}$. Furthermore $p \in J_{g}$ and $m \in \hat{J}_{g}$. It is easily seen that if $j_{\alpha}=p$ we have

$$
\begin{aligned}
\hat{\jmath}_{r} & =j_{r}, \quad 1 \leq r \leq \alpha-1, \\
\hat{\jmath}_{r}+1 & =j_{r+1}, \quad \alpha \leq r \leq d-1, \\
\hat{\jmath}_{d} & =m .
\end{aligned}
$$

We next prove that

$$
\mathfrak{h}(g, \mathscr{F})=\mathfrak{h}(g, \hat{\mathscr{F}}) .
$$

We start by observing that $\left(\mathfrak{g}_{j}\right)_{g_{j}} \subseteq \mathfrak{k}$ for $1 \leq j \leq m$. In fact, let $\gamma: \mathfrak{g} \rightarrow \mathbf{R}$ be the linear map such that $\left[X, X_{2}\right]=\gamma(X) X_{1}$. Then $X \in \mathfrak{k} \Leftrightarrow \gamma(X)=0$. If $j=1$, then clearly $\left(\mathfrak{g}_{j}\right)_{g_{j}} \subseteq \mathfrak{k}$. Suppose $j \geq 2$. Then $X_{2} \in \mathfrak{g}_{j}$, and if $X \in\left(\mathfrak{g}_{j}\right)_{g_{j}}$, then $0=\left\langle g,\left[X, X_{2}\right]\right\rangle=\gamma(X)\left\langle g, X_{1}\right\rangle$ which implies that $\gamma(X)=0$, hence $X \in \mathfrak{k}$. This shows that $\left(\mathfrak{g}_{j}\right)_{g_{j}} \subseteq \mathfrak{k}$ for all $1 \leq j \leq m$. Now for $1 \leq j<p$ we have $\hat{\mathfrak{g}}_{j}=\mathfrak{g}_{j}$ and therefore $\left(\hat{\mathfrak{g}}_{j}\right)_{\hat{\mathfrak{g}}_{j}}=\left(\mathfrak{g}_{j}\right)_{g_{j}}$. For $p-1 \leq j \leq m-1$ we have $\hat{\mathfrak{g}}_{j}=\mathfrak{g}_{j+1} \cap \mathfrak{k}$. Therefore, if $X \in\left(\mathfrak{g}_{j+1}\right)_{g_{j+1}}$ we have that $X \in \mathfrak{k}$, hence $X \in \hat{\mathfrak{g}}_{j}$, and $0=\left\langle g,\left[X, \mathfrak{g}_{j+1}\right]\right\rangle \supset\left\langle g,\left[X, \hat{\mathfrak{g}}_{j}\right]\right\rangle$ which shows that $X \in\left(\hat{\mathfrak{g}}_{j}\right)_{\hat{g}_{j}}$. We conclude that $\left(\mathfrak{g}_{j+1}\right)_{g_{j+1}} \subset\left(\hat{\mathfrak{g}}_{j}\right)_{\hat{g}_{j}}$. But then $\mathfrak{h}(g, \mathscr{F})=\sum_{j=1}^{m}\left(\mathfrak{g}_{j}\right)_{g_{j}}=\sum_{j=1}^{p-1}\left(\mathfrak{g}_{j}\right)_{g_{j}}+$ $\sum_{j=p}^{m}\left(\mathfrak{g}_{j}\right)_{g_{j}}=\sum_{j=1}^{p-1}\left(\hat{g}_{j}\right)_{\hat{g}_{j}}+\sum_{j=p-1}^{m-1}\left(\mathfrak{g}_{j+1}\right)_{g_{j+1}} \subset \sum_{j=1}^{p-1}\left(\hat{\mathfrak{g}}_{j}\right)_{\hat{g}_{j}}+\sum_{j=p-1}^{m-1}\left(\hat{\mathfrak{g}}_{j}\right)_{\hat{\mathrm{g}}_{j}}=$ $\sum_{j=1}^{m-1}\left(\hat{\mathfrak{g}}_{j}\right)_{\hat{g}_{j}} \subset \sum_{j=1}^{m}\left(\hat{\mathfrak{g}}_{j}\right)_{\hat{g}_{j}}=\mathfrak{h}(O, \hat{\mathscr{F}})$. This shows that $\mathfrak{h}(g, \mathscr{F}) \subset \mathfrak{h}(O, \hat{F})$, since $\operatorname{dim} \mathfrak{h}(g, \mathscr{F})=\operatorname{dim} \mathfrak{h}(O, \hat{F})(=d / 2)$ we have that $\mathfrak{h}(g, \mathscr{F})=\mathfrak{h}(O, \hat{\mathscr{F}})$.

We shall then compare the $\mathscr{B}$-chart $\sigma_{O}^{\mathscr{B}}$ and the $\hat{\mathscr{B}}$-chart $\sigma_{O}^{\hat{\mathscr{B}}}$ : For $1 \leq r \leq$ $\alpha-1$ we have $\sigma_{O}^{\mathscr{B}}(l)_{r}=\left\langle l, X_{j_{r}}\right\rangle=\left\langle l, X_{j_{r}}\right\rangle=\left\langle l, \hat{X}_{j_{r}}\right\rangle=\sigma_{O}^{\mathscr{B}}(l)_{r}$. For $\alpha \leq r \leq$ $d-1$ we have $\sigma_{O}^{\mathscr{B}}(l)_{r}=\left\langle l, \hat{X}_{j_{r}}\right\rangle=\left\langle l, X_{j_{r}+1}+c_{\hat{\jmath}_{r}+1} X_{p}\right\rangle=\left\langle l, X_{j_{r+1}}+c_{j_{r+1}} X_{p}\right\rangle=$ $\sigma_{O}^{\mathscr{B}}(l)_{r+1}+c_{j_{r+1}} \sigma_{O}^{\mathscr{B}}(l)_{\alpha}$. Finally $\sigma_{O}^{\mathscr{B}}(l)_{\alpha}=\left\langle l, X_{j_{\alpha}}\right\rangle=\left\langle l, X_{p}\right\rangle^{r+1}=\left\langle l, \hat{X}_{m}\right\rangle=$ 
$\sigma_{O}^{\hat{\mathscr{B}}}(l)_{m}$. In other words, if $\sigma_{O}^{\mathscr{B}}(l)=\left(y_{1}, \ldots, y_{d}\right)$, and $\sigma_{O}^{\hat{\mathscr{B}}}(l)=\left(\hat{y}_{1}, \ldots, \hat{y}_{d}\right)$ we have

$$
\begin{aligned}
& \hat{y}_{1}=y_{1} \\
& \vdots \\
& \hat{y}_{\alpha-1}=y_{\alpha-1} \\
& \hat{y}_{\alpha}=y_{\alpha+1}+c_{j_{\alpha+1}} y_{\alpha} \\
& \vdots \\
& \hat{y}_{r}=y_{r+1}+c_{j_{r+1}} y_{\alpha} \\
& \vdots \\
& \hat{y}_{d-1}=y_{d}+c_{j_{d}} y_{\alpha} \\
& \hat{y}_{d}=y_{\alpha} .
\end{aligned}
$$

Let now $\varphi \in \mathscr{P}_{F(O, \mathscr{F})}^{0}(O)=\mathscr{P}_{F(O, \hat{F})}^{0}(O)$. With the above notation we have $\varphi \circ \sigma_{O}^{\hat{\mathscr{B}}^{-1}}(\hat{y})=\varphi \circ \sigma_{O}^{\mathscr{B}}-1(y)$. We now assert that $\Phi_{O}^{\mathscr{B}}(\varphi)=\Phi_{O}^{\hat{\mathscr{B}}}(\varphi)$. But this is clear since

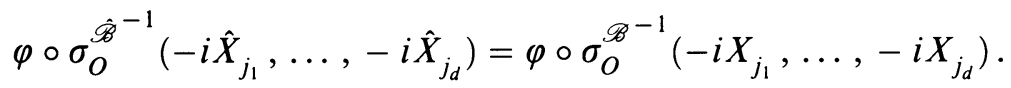

From now on we then assume that $\mathfrak{g}_{m-1}=\mathfrak{k}=\operatorname{ker}\left(\operatorname{ad} X_{2}\right)$, and set $g_{0}=$ $g|\mathfrak{k}=g| \mathfrak{g}_{m-1}$. Finally we set $K=\exp \mathfrak{k}$.

Set $K_{g}=\left\{1 \leq j \leq m \mid X_{j} \notin \mathfrak{g}_{j-1}+\mathfrak{h}(g, \mathscr{F})\right\}$. Then writing $K_{g}=\left\{k_{1}<\right.$ $\left.\ldots<k_{d / 2}\right\}$ we have that $X_{k_{1}}, \ldots, X_{k_{d / 2}}$ is a basis of $\mathfrak{g}(\bmod \mathfrak{h}(g, \mathscr{F}))$ of subalgebra type. Since $\mathfrak{h}(g, \mathscr{F}) \subset \mathfrak{k}=\mathfrak{g}_{m-1}$ we have that $k_{d / 2}=m$. Using this basis we have a unitary $V: L^{2}(G, \chi) \rightarrow L^{2}\left(\mathbf{R}^{d / 2}, d t\right)$, and it maps $\mathscr{S}(G, \chi)$ onto $\mathscr{S}\left(\mathbf{R}^{d / 2}\right)$, see $\S 1.8$. Set $\pi=\pi_{\chi}=$ ind $_{H \uparrow G} \chi$. The representation $\pi$ is transformed into the representation $\pi^{V}$ having the form

$$
\pi^{V}(s) h(t)=\chi(b(s, t))^{-1} h\left(s^{-1} t\right),
$$

see $\S 1.8$.

In the following we indicate objects defined relative to the subgroup $K$ by a subscript or superscript " 0 ". In $\mathfrak{k}$ we have a Jordan-Hölder basis $\mathscr{B}_{0}: X_{1}, \ldots$, $X_{m-1}$. We call the associated Jordan-Hölder sequence $\mathscr{F}_{0}$. Clearly $\mathfrak{h}(g, \mathscr{F})=$ $\mathfrak{h}\left(g_{0}, \mathscr{F}_{0}\right)$, where $g_{0}=g \mid \mathfrak{k}$. We have $\mathfrak{k}_{g_{0}}=\mathfrak{g}_{g} \oplus \mathbf{R} X_{2}$ and writing $J_{g_{0}}=$ $\left\{j_{1}^{0}<\cdots<j_{d-2}^{0}\right\}$ we have that $j_{r}^{0}=j_{r+1}, r=1, \ldots, d-2$. Moreover, $X_{k_{1}}, \ldots, X_{k_{d / 2-1}}$ is a basis in $\mathfrak{k}\left(\bmod \mathfrak{h}\left(g_{0}, \mathscr{F}_{0}\right)\right)$ of subalgebra type. We set $\pi_{0}=$ ind $_{H \uparrow K} \chi$, and transform $\pi_{0}$ to $\pi_{0}^{V_{0}}$ acting on $L^{2}\left(\mathbf{R}^{d / 2-1}, d t^{0}\right)$ by the unitary $V_{0}$, just as we did with $\pi=\pi_{\chi}$, and we get

$$
\pi_{0}^{V_{0}}\left(s_{0}\right) h_{0}\left(t^{0}\right)=\chi\left(b_{0}\left(s_{0}, t^{0}\right)\right)^{-1} h_{0}\left(s_{0}^{-1} t^{0}\right) \text {. }
$$


We identify $\mathbf{R}^{d / 2}$ with $\mathbf{R}^{d / 2-1} \times \mathbf{R}$ as follows $t=\left(t^{0}, t_{d / 2}\right)$. A simple calculation then gives that

$$
\begin{aligned}
& d \pi^{V}\left(X_{m}\right) f(t)=-\frac{\partial f}{\partial t_{d / 2}}(t), \\
& d \pi^{V}(X) f(t)=d \pi_{0}^{V_{0}}\left(e^{-t_{d / 2} \mathrm{ad} X_{m}} X\right) f\left(t^{0}, t_{d / 2}\right)
\end{aligned}
$$

for $X \in \mathfrak{k}$, where $d \pi_{0}^{V_{0}}\left(e^{-t_{d / 2} \text { ad } X_{m}} X\right)$ acts in the first variable.

Let us now note that if $l=s g \in O$, and $y=\sigma_{O}^{\mathscr{B}}(l)$, then $\left\langle s \exp u X_{2} g, X_{j}\right\rangle=$ $\left\langle s g, X_{j}\right\rangle$ for $j<m$ while

$$
\begin{aligned}
\left\langle s \exp u X_{2} g, X_{m}\right\rangle & =\left\langle s g, e^{-u \operatorname{ad} X_{2}} X_{m}\right\rangle=\left\langle s g, X_{m}+u\left[X_{m}, X_{2}\right]\right\rangle \\
& =\left\langle s g, X_{m}\right\rangle+u\left\langle g,\left[X_{m}, X_{2}\right]\right\rangle=\left\langle l, X_{m}\right\rangle+u \mu,
\end{aligned}
$$

where $\mu=\left\langle g,\left[X_{m}, X_{2}\right]\right\rangle$. It follows that $\varphi \circ{\sigma_{O}^{\mathscr{B}}}^{-1}\left(y_{1}, \ldots, y_{d-1}, y_{d}+u \mu\right)=$ $\varphi\left(s \exp u X_{2} g\right)=\varphi(s g)=\varphi \circ \sigma_{O}^{\mathscr{B}}-1\left(y_{1}, \ldots, y_{d-1}, y_{d}\right)$, since $\varphi \in \mathscr{P}_{F(O, \mathscr{F})}^{0}(O)$ and $X_{2} \in \mathfrak{h}(g, \mathscr{F})$. The conclusion is that $\varphi \circ \sigma_{O}^{\mathscr{B}}-1$ only depends on the variables $y_{1}, \ldots, y_{d-1}$, and therefore $\varphi \circ \sigma_{O}^{\mathscr{B}}-1\left(-i X_{j_{1}}, \ldots,-i X_{j_{d}}\right) \in S\left(\mathfrak{k}_{\mathbf{C}}\right)$, hence $\Phi_{O}^{\mathscr{B}}(\varphi) \in U\left(\mathfrak{k}_{\mathrm{C}}\right)$. It also follows that there exists a function $\bar{\varphi}$ on $\bar{O}=$ $\{l|\mathfrak{k}| l \in O\}$ such that $\varphi(l)=\bar{\varphi}(l \mid \mathfrak{k})$ for all $l \in O$, and $\bar{\varphi}$ is the restriction to $\bar{O}$ of a polynomial function.

We write

$$
\varphi \circ \sigma_{O}^{\mathscr{B}-1}\left(y_{1}, \ldots, y_{d}\right)=\sum_{\alpha=\left(\alpha_{2}, \ldots, \alpha_{d-1}\right)} a_{\alpha}\left(y_{1}\right) y_{2}^{\alpha_{2}} \cdots y_{d-1}^{\alpha_{d-1}},
$$

where $a_{\alpha}\left(y_{1}\right)$ is a polynomial function in $y_{1}$.

Set $O_{0}=K g_{0}$, and set $\varphi_{0}=\bar{\varphi} \mid O_{0}$. Then if $l_{0} \in O_{0}$ and $l \in O$ with $l \mid k=l_{0}$ we have $\left\langle l_{0}, X_{2}\right\rangle=\xi_{2}=\left\langle g, X_{2}\right\rangle$. It follows that if $\sigma_{O_{0}}^{\mathscr{B}_{0}}\left(l_{0}\right)=\left(y_{2}, \ldots, y_{d-1}\right)$, then $\sigma_{O}^{\mathscr{B}}(l)=\left(\xi_{2}, y_{2}, \ldots, y_{d-1}, y_{d}\right)$, and therefore that

$$
\begin{gathered}
\varphi \circ \sigma_{O}^{\mathscr{B}-1}\left(\xi_{2}, y_{2}, \ldots, y_{d-1}, y_{d}\right)=\varphi(l)=\varphi_{0}\left(l_{0}\right) \\
=\varphi_{0} \circ \sigma_{O_{0}}^{\mathscr{B}_{0}-1}\left(y_{2}, \ldots, y_{d-1}\right) .
\end{gathered}
$$

The conclusion is that

$$
\varphi_{0} \circ \sigma_{O_{0}}^{\mathscr{B}_{0}-1}\left(y_{2}, \ldots, y_{d-1}\right)=\sum_{\alpha=\left(\alpha_{2}, \ldots, \alpha_{d-1}\right)} a_{\alpha}\left(\xi_{2}\right) y_{2}^{\alpha_{2}} \cdots y_{d-1}^{\alpha_{d-1}}
$$

Now

$$
\begin{aligned}
\Phi_{O}^{\mathscr{B}}(\varphi) & =\omega\left(\varphi \circ \sigma_{O}^{\mathscr{B}-1}\left(-i X_{j_{1}}, \ldots,-i X_{j_{d}}\right)\right) \\
& =\omega\left(\sum_{\alpha} a_{\alpha}\left(-i X_{2}\right)\left(-i X_{j_{2}}\right)^{\alpha_{2}} \cdots\left(-i X_{j_{d-1}}\right)^{\alpha_{d-1}}\right) \\
& =\sum_{\alpha} a_{\alpha}\left(-i X_{2}\right) \omega\left(\left(-i X_{j_{2}}\right)^{\alpha_{2}} \cdots\left(-i X_{j_{d-1}}\right)^{\alpha_{d-1}}\right) .
\end{aligned}
$$


Here we have used that $X_{2}$ is central in $\mathfrak{k}$. But then

$$
\begin{aligned}
d \pi_{0}\left(\Phi_{O}^{\mathscr{B}}(\varphi)\right) & =\sum_{\alpha} d \pi_{0}\left(a_{\alpha}\left(-i X_{2}\right)\right) d \pi_{0}\left(\omega\left(\left(-i X_{j_{2}}\right)^{\alpha_{2}} \cdots\left(-i X_{j_{d-1}}\right)^{\alpha_{d-1}}\right)\right) \\
& =\sum_{\alpha} a_{\alpha}\left(\xi_{2}\right) d \pi_{0}\left(\omega\left(\left(-i X_{j_{1}^{0}}\right)^{\alpha_{2}} \cdots\left(-i X_{j_{d-2}^{0}}\right)^{\alpha_{d-1}}\right)\right) \\
& =d \pi_{0}\left(\omega\left(\sum_{\alpha} a_{\alpha}\left(\xi_{2}\right)\left(-i X_{j_{1}^{0}}\right)^{\alpha_{2}} \cdots\left(-i X_{j_{d-2}^{0}}\right)^{\alpha_{d-1}}\right)\right. \\
& =d \pi_{0}\left(\omega\left(\varphi_{0} \circ \sigma_{O_{0}}^{\mathscr{B}_{0}}{ }^{-1}\left(-i X_{j_{1}^{0}}, \cdots,-i X_{j_{d-2}^{0}}\right)\right)\right) \\
& =d \pi_{0}\left(\Phi_{O_{0}}^{\mathscr{B}_{0}}\left(\varphi_{0}\right)\right) .
\end{aligned}
$$

By the induction hypothesis we have that $d \pi_{0}\left(\Phi_{O_{0}}^{\mathscr{B}_{0}}\left(\varphi_{0}\right)\right)=\delta_{\chi}^{0}\left(\varphi_{0}\right)$, and therefore we get

$$
d \pi_{0}\left(\Phi_{O}^{\mathscr{B}}(\varphi)\right)=\delta_{\chi}^{0}\left(\varphi_{0}\right)
$$

We shall now find $\operatorname{Ad}\left(\exp -t X_{m}\right)\left(\Phi_{O}^{\mathscr{B}}(\varphi)\right)$ for $t \in \mathbf{R}$. First, setting $X_{j}^{t}=$ $\operatorname{Ad}\left(\exp -t X_{m}\right) X_{j}, \quad 1 \leq j \leq m$, we get a new Jordan-Hölder basis $\mathscr{B}^{t}$ compatible with the Jordan-Hölder sequence $\mathscr{F}$. Applying the formula $(*)$ to the Jordan-Hölder basis $\mathscr{B}^{t}$ we get

$$
d \pi_{0}\left(\Phi_{O}^{\mathscr{B}^{t}}(\varphi)\right)=\delta_{\chi}^{0}\left(\varphi_{0}\right)
$$

Next, define the function $\varphi_{t}$ by $\varphi_{t}(l)=\varphi\left(\exp t X_{m} l\right)$. Clearly $\varphi_{t} \in \mathscr{P}_{F(O, \mathscr{F})}^{0}(O)$. If $\varphi \circ \sigma_{O}^{\mathscr{B}^{-1}}=\sum_{\alpha} c_{\alpha} y^{\alpha}$, then

$$
\varphi(l)=\sum_{\alpha} c_{\alpha}\left(\left\langle l, X_{j_{1}}\right\rangle\right)^{\alpha_{1}} \cdots\left(\left\langle l, X_{j_{d}}\right\rangle\right)^{\alpha_{d}}
$$

and therefore

$$
\begin{aligned}
\varphi_{t}(l) & =\varphi\left(\exp t X_{m} l\right)=\sum_{\alpha} c_{\alpha}\left(\left\langle\exp t X_{m} l, X_{j_{\alpha}}\right\rangle\right)^{\alpha} \\
& =\sum_{\alpha} c_{\alpha}\left(\left\langle l, \operatorname{Ad}\left(\exp -t X_{m}\right) X_{j_{1}}\right\rangle\right)^{\alpha_{1}} \cdots\left(\left\langle l, \operatorname{Ad}\left(\exp -t X_{m}\right) X_{j_{d}}\right\rangle\right)^{\alpha_{d}} \\
& =\sum_{\alpha} c_{\alpha}\left(\left\langle l, X_{j_{1}}^{t}\right\rangle\right)^{\alpha_{1}} \cdots\left(\left\langle l, X_{j_{d}}^{t}\right\rangle\right)^{\alpha_{d}} .
\end{aligned}
$$

Now we see from above that $\varphi_{t} \circ \sigma_{O}^{\mathscr{B}^{t}-1}=\varphi \circ \sigma_{O}^{\mathscr{B}}-1$, so

$$
\begin{aligned}
& \varphi_{t} \circ \sigma_{O}^{\mathscr{B}^{t}-1}\left(-i X_{j_{1}}^{t}, \ldots,-i X_{j_{d}}^{t}\right)=\varphi \circ{\sigma_{O}^{\mathscr{B}}}^{-1}\left(-i X_{j_{1}}^{t}, \ldots,-i X_{j_{d}}^{t}\right) \\
& =\operatorname{Ad}\left(\exp -t X_{m}\right)\left(\varphi \circ \sigma_{O}^{\mathscr{B}-1}\left(-i X_{j_{1}}, \ldots,-i X_{j_{d}}\right)\right),
\end{aligned}
$$

which implies that

$$
\Phi_{O}^{\mathscr{B}^{\prime}}\left(\varphi_{t}\right)=\operatorname{Ad}\left(\exp -t X_{m}\right)\left(\Phi_{O}^{\mathscr{B}}(\varphi)\right)
$$


Using $(* *)$ and $(* * *)$ we then get

$$
d \pi_{0}\left(\operatorname{Ad}\left(\exp -t X_{m}\right) \Phi_{O}^{\mathscr{B}}(\varphi)\right)=\delta_{\chi}^{0}\left(\left(\varphi_{t}\right)_{0}\right)
$$

But then it follows that

$$
\begin{aligned}
d \pi^{V} & \left(\Phi_{O}^{\mathscr{B}}(\varphi)\right) f(t)=d \pi_{0}^{V_{0}}\left(\operatorname{Ad}\left(\exp -t_{d / 2} X_{m}\right) \Phi_{O}^{\mathscr{B}}(\varphi)\right) f(t) \\
& =\delta_{\chi}^{0 V_{0}}\left(\left(\varphi_{t_{d / 2}}\right)_{0}\right) f\left(t^{0}, t_{d / 2}\right) \\
& =i \varphi_{t_{d / 2}}\left(\exp t_{d / 2-1} X_{k_{d / 2-1}} \cdots \exp t_{1} X_{k_{1}} g\right) f(t) \\
& =i \varphi\left(\exp t_{d / 2} X_{m} \exp t_{d / 2-1} X_{k_{d / 2-1}} \cdots \exp t_{1} X_{k_{1}} g\right) f(t) \\
& =\delta_{\chi}^{V}(\varphi) f(t)
\end{aligned}
$$

and therefore

$$
d \pi\left(\Phi_{O}^{\mathscr{B}}(\varphi)\right)=\delta_{\chi}(\varphi)
$$

This finishes Case (b), and ends the proof of Theorem 3.1.2.

We have till now only defined $\Phi(\varphi) \in U\left(\mathfrak{g}_{\mathbf{C}}\right)$ for $\varphi \in \mathscr{P}_{F(O, \mathscr{F})}^{0}(O)$. We shall now define $\Phi(\varphi) \in U\left(\mathfrak{g}_{\mathrm{C}}\right)$ for $\varphi \in \mathscr{P}_{F(O, \mathscr{F})}^{1}(O)$. To this end, recall from Proposition 2.2.9 that each $\varphi \in \mathscr{P}_{F(O, \mathscr{F})}^{1}(O)$ can be written uniquely as

$$
\varphi=\sum_{r=1}^{d / 2} \varphi_{r} \psi^{X_{k_{r}}}+\varphi_{0},
$$

where $\varphi_{0}, \varphi_{1}, \ldots, \varphi_{d / 2}$ belong to $\mathscr{P}_{F(O, \mathscr{F})}^{0}(O)$. We set

$$
\Phi(\varphi)=-i \sum_{r=1}^{d / 2} \Phi\left(\varphi_{r}\right) X_{k_{r}}+\Phi\left(\varphi_{0}\right)
$$

Theorem 3.1.3. For $\varphi \in \mathscr{P}_{F(O, \mathscr{F})}^{1}(O)$ we have

$$
d \pi_{\chi}(\Phi(\varphi))=\delta_{\chi}(\varphi)
$$

Proof. Since $\delta_{\chi}$ is $\mathscr{P}_{F}^{0}(O)$-linear this follows immediately from Theorem 3.1.2 and the definition of $\Phi$.

We now consider the map $\varepsilon_{\chi}$. Recall (see $\left.\S 2.5\right)$ that $\varepsilon_{\chi}$ defines an isomorphism

$$
\varepsilon_{\chi}: \mathscr{P}_{F}^{1}(O) \rightarrow \mathscr{A}^{1}(G, \chi)
$$

We set $\tilde{\Psi}$ to be the map that makes the following diagram commutative:

$$
\begin{aligned}
& \mathscr{P}_{F}^{1}(O) \stackrel{\dot{\Psi}}{\longrightarrow} J_{F}^{1}(O) / I(O) \\
& \varepsilon_{\chi} \searrow \quad \swarrow d \pi \\
& \mathscr{A}^{1}(G, \chi)
\end{aligned}
$$


We shall now construct an explicit map $\Psi: \mathscr{P}_{F}^{1}(O) \rightarrow \mathscr{A}^{1}(G, \chi)$ such that $\Psi$ when composed with the quotient map $J_{F}^{1}(O) \rightarrow J_{F}^{1}(O) / I(O)$ is equal to $\tilde{\Psi}$, just as we did for $\tilde{\Phi}$ :

We set $\Psi(\varphi)=\Phi(\varphi)$ for $\varphi \in \mathscr{P}_{F(O, \mathscr{F})}^{0}(O)$. For $\varphi \in \mathscr{P}_{F}^{1}(O)$ we write $\varphi$ as in the formula $(\sharp)$ above, and set

$$
\Psi(\varphi)=-i \frac{1}{2} \sum_{r=1}^{d / 2}\left(\Psi\left(\varphi_{r}\right) X_{k_{r}}+X_{k_{r}} \Psi\left(\varphi_{r}\right)\right)+\Psi\left(\varphi_{0}\right) .
$$

Theorem 3.1.4. For $\varphi \in \mathscr{P}_{F(O, \mathscr{F})}^{1}(O)$ we have

Proof. We have

$$
d \pi_{\chi}(\Psi(\varphi))=\varepsilon_{\chi}(\varphi)
$$

$$
\begin{aligned}
d \pi_{\chi}(\Psi(\varphi)) & =-i \frac{1}{2} \sum_{r=1}^{d / 2}\left(d \pi\left(\Psi\left(\varphi_{r}\right)\right) d \pi\left(X_{k_{r}}\right)+d \pi\left(X_{k_{r}}\right) d \pi\left(\Psi\left(\varphi_{r}\right)\right)\right)+d \pi\left(\Psi\left(\varphi_{0}\right)\right) \\
& =-i \frac{1}{2} \sum_{r=1}^{d / 2}\left(\delta_{\chi}\left(\varphi_{r}\right) d \pi\left(X_{k_{r}}\right)+d \pi\left(X_{k_{r}}\right) \delta_{\chi}\left(\varphi_{r}\right)\right)+\delta_{\chi}\left(\varphi_{0}\right) .
\end{aligned}
$$

Now

$$
\delta_{\chi}(\varphi)=-i \sum_{r=1}^{d / 2} \delta_{\chi}\left(\varphi_{r}\right) d \pi\left(X_{k_{r}}\right)+\delta_{\chi}\left(\varphi_{0}\right)
$$

hence

$$
\delta_{\chi}(\bar{\varphi})^{*}=i \sum_{r=1}^{d / 2} d \pi\left(X_{k_{r}}\right) \delta_{\chi}\left(\varphi_{r}\right)-\delta_{\chi}\left(\varphi_{0}\right)
$$

We see that the difference of the last two expressions is equal to twice the result of the first calculation, hence

$$
\varepsilon_{\chi}(\varphi)=\frac{1}{2}\left(\delta_{\chi}(\varphi)-\delta_{\chi}(\bar{\varphi})^{*}\right)=d \pi(\Psi(\varphi)) .
$$

This ends the proof of the theorem.

3.2. Construction of unitary representations with an explicit cross-section for the differential. Suppose now that $\left(p_{1}, \ldots, p_{d / 2}, q_{1}, \ldots, q_{d / 2}\right)$ are global canonical coordinates on $O$ such that $p_{1}, \ldots, p_{d / 2} \in \mathscr{P}_{F(O, F)}^{1}(O)$ and $q_{1}, \ldots, q_{d / 2} \in \mathscr{P}_{F(O, \mathscr{F})}^{0}(O)$. As in $\S 2.7$ we write for each $X \in \mathfrak{g}$

$$
\psi^{X}=\sum_{r=1}^{d / 2} a_{X, r}(q) p_{r}+a_{X, 0}(q)
$$

where $a_{X, r} \in \mathscr{P}\left(\mathbf{R}^{d / 2}\right), r=0,1, \ldots, d / 2$. 
We set $u_{r}=\Psi\left(p_{r}\right), v_{r}=\Psi\left(q_{r}\right), r=1, \ldots, d / 2$. We can then formulate:

Theorem 3.2.1 There exists one and only one strongly continuous, unitary representation $\pi$ of $G$ on $L^{2}\left(\mathbf{R}^{d / 2}\right)$ such that $\mathscr{D}\left(\mathbf{R}^{d / 2}\right) \subset H_{\pi}^{\infty}$, and such that

$$
d \pi(X) f(t)=\sum_{r=1}^{d / 2} a_{X, r}(t) \frac{\partial f}{\partial t_{r}}(t)+i a_{X, 0}(t) f(t)+\frac{1}{2} \sum_{r=1}^{d / 2} \frac{\partial a_{X, r}}{\partial t_{r}}(t) f(t)
$$

for $f \in \mathscr{D}\left(\mathbf{R}^{d / 2}\right)$. The representation $\pi$ is in the equivalence class of irreducible representations associated with the orbit $O$, and we have

$$
d \pi\left(u_{r}\right)=D_{r}, \quad d \pi\left(v_{r}\right)=i M_{r},
$$

where $D_{r} f=\partial f / \partial t_{r}$ and $M_{r} f(t)=t_{r} f(t), r=1, \ldots, d / 2$.

Proof. This follows from Theorem 3.1.4 and [9, Theorem 3.2.3] (and also invoking a few details from proof of this theorem).

\section{CoARse AND Fine $\mathscr{F}$-STRATIFICATION OF THE DUAL OF A NILPOTENT LIE ALGEBRA}

The main purpose of this section is to define the "fine $\mathscr{F}$-stratification"of the dual of a nilpotent Lie algebra with respect to a given Jordan-Hölder sequence $\mathscr{F}$. This will be used in an essential way in $\S 5$. The fine $\mathscr{F}$-stratification is a refinement of what we call the "coarse $\mathscr{F}$-stratification", which is due to Pukanszky.

4.1. Jump indices for skewsymmetric bilinear form with respect to flag. We start by defining the "jump indices"of a skewsymmetric bilinear form.

Let $V$ be a finite dimensional vector space over the field $\mathbf{K}$, and let $B: V \times$ $V \rightarrow \mathbf{K}$ be a skewsymmetric bilinear form on $V$. If $M$ is any subset we denote by $M^{\perp}$ the set $M^{\perp}=\{v \in V \mid B(u, v)=0$ for all $u \in M\}$. We define the radical $V_{B}$ of $B$ to be the subspace $V^{\perp}$.

Suppose that

$$
\mathscr{F}: V=V_{m} \supset V_{m-1} \supset \cdots \supset V_{1} \supset V_{0}=\{0\}
$$

is a flag of subspaces, i.e. an increasing sequence of subspaces such that $\operatorname{dim} V_{j}$ $=j$.

Set

$$
J_{B}=\left\{1 \leq j \leq m \mid V_{j} \not \subset V_{j-1}+V_{B}\right\} .
$$

We call $J_{B}$ the set of jump indices of $B$ relative to the given flag $\mathscr{F}$. have

Let $x_{j} \in V_{j} \backslash V_{j-1}, j=1, \ldots, m$. Then $x_{1}, \ldots, x_{m}$ is a basis in $V$. We

$$
J_{B}=\left\{1 \leq j \leq m \mid x_{j} \notin V_{j-1}+V_{B}\right\} .
$$

If $J_{B}$ is nonempty we can write $J_{B}=\left\{j_{1}<\cdots<j_{d}\right\}$. Then $x_{j_{1}}, \ldots, x_{j_{d}}$ is a basis for $V\left(\bmod V_{B}\right)$. This implies that the matrix

is nonsingular.

$$
M_{B}=\left[B\left(x_{j_{u}}, x_{j_{v}}\right)\right]_{1 \leq u, v \leq d}
$$


Set $B_{j}$ to be the restriction of $B$ to $V_{j} \times V_{j}, j=1, \ldots, m$. The symbol $\left(V_{j}\right)_{B_{j}}$ then denotes the radical of $B_{j}$. For the set of jump indices $J_{B_{j}}$ of $B_{j}$ relative to the flag

$$
\mathscr{F}_{j}: V_{j} \supset V_{j-1} \supset \cdots \supset V_{1} \supset V_{0}=\{0\}
$$

we have

$$
J_{B_{j}}=\left\{1 \leq k \leq j \mid x_{k} \notin V_{k-1}+\left(V_{j}\right)_{B_{j}}\right\} .
$$

Let us note that $J_{B_{j}} \subset J_{B}$; in fact, we clearly have that $J_{B}=\{1 \leq j \leq m \mid$ $\left.x_{j} \notin V_{j-1}+V_{B} \cap V_{j}\right\}$, and also $V_{B} \cap V_{j} \subset\left(V_{j}\right)_{B j}$; it follows that $x_{k} \in V_{k-1}+V_{B} \cap$ $V_{j} \Rightarrow x_{k} \in V_{k-1}+\left(V_{j}\right)_{B_{j}}$, and therefore $x_{k} \notin V_{k-1}+\left(V_{j}\right)_{B_{j}} \Rightarrow x_{k} \notin V_{k-1}+V_{B} \cap V_{j}$. This implies that $J_{B_{j}} \subset J_{B}$. In general we have

$$
\varnothing=J_{B_{1}} \subset J_{B_{2}} \subset \cdots \subset J_{B_{m-1}} \subset J_{B_{m}}=J_{B} .
$$

Set

$$
K_{B}=\left\{1 \leq j \leq m \mid J_{B_{j-1}} \neq J_{B_{j}}\right\}
$$

Now either

$$
\operatorname{dim} V_{j} /\left(V_{j}\right)_{B_{j}}=\operatorname{dim} V_{j-1} /\left(V_{j-1}\right)_{B_{j-1}}
$$

or

$$
\operatorname{dim} V_{j} /\left(V_{j}\right)_{B_{j}}=\operatorname{dim} V_{j-1} /\left(V_{j-1}\right)_{B_{j-1}}+2
$$

$[3,1.12 .2]$. It follows that either $\operatorname{card}\left(J_{B_{j}}\right)=\operatorname{card}\left(J_{B_{j-1}}\right)$ or $\operatorname{card}\left(J_{B_{j}}\right)=$ $\operatorname{card}\left(J_{B_{j-1}}\right)+2$. Since $J_{B_{1}}=\varnothing$ and $\operatorname{card}\left(J_{B_{m}}\right)=\operatorname{card}\left(J_{B}\right)=d$ is even we get that $\operatorname{card}\left(K_{B}\right)=d / 2$.

Another characterization of $K_{B}$ is

$$
K_{B}=\left\{1 \leq j \leq m \mid j \in J_{B_{j}}\right\} ;
$$

in fact, if $j \in J_{B_{j}}$, then $j \notin J_{B_{j-1}}$, so $j \in K_{B}$. Conversely, suppose that $j \notin J_{B_{j}}$. Then $x_{j} \in V_{j-1}+\left(V_{j}\right)_{B_{j}}$ and therefore $\left(V_{B_{j}}\right) \cap V_{j-1}=\left(V_{j-1}\right)_{B_{j-1}}$, hence $J_{B_{j-1}}=J_{B_{j}}$. This proves (*). In particular we get $j \in K_{B} \Rightarrow j \in J_{B_{j}} \subset J_{B}$, so $K_{B} \subset J_{B}$.

Still another characterization of $K_{B}$ is as follows: We know that $P(B, \mathscr{F})=$ $\sum_{j=1}^{m}\left(V_{j}\right)_{B_{j}}$ is a maximal isotropic subspace in $V$ with respect to $B$, and that $P(B, \mathscr{F}) \cap V_{j}=P\left(B_{j}, \mathscr{F}_{j}\right)[3,1.12 .3]$. From this we see immediately that

$$
K_{B}=\left\{1 \leq j \leq m \mid V_{j} \not \subset V_{j-1}+P(B, \mathscr{F})\right\} .
$$

We set

$$
\mathscr{J}_{B}=\left(J_{B_{1}}, J_{B_{2}}, \ldots, J_{B_{m-1}}, J_{B_{m}}\right)
$$


Definition 4.1.1. We call $\mathscr{J}_{B}$ for the $m$-tuple of jump indices for $B$ with respect to the given flag $\mathscr{F}$.

We write

$$
J_{B}=\left\{j_{1}<\cdots<j_{d}\right\}, \quad K_{B}=\left\{k_{1}<\cdots<k_{d / 2}\right\} .
$$

If $j<k_{1}$ we have $J_{B_{j}}=\varnothing$ and if $j>k_{d / 2}$ we have $J_{B_{j}}=J_{B}$. For $k_{r} \leq j<$ $k_{r+1}$ we have $J_{B_{k_{r}}}=J_{B_{j}} \neq J_{B_{k_{r+1}}}$.

We define the finite sequence $l_{1}, \ldots, l_{d / 2}$ in $\{1, \ldots, m\}$ recursively as follows

$$
J_{B_{k_{1}}}=\left\{l_{1}<k_{1}\right\}, \quad J_{B_{k_{r+1}}}=J_{B_{k_{r}}} \cup\left\{l_{r+1}<k_{r+1}\right\}, \quad r<d / 2-1,
$$

and we set $L_{B}=\left\{l_{1}, \ldots, l_{d / 2}\right\}$. Then $J_{B}=K_{B} \cup L_{B}$ as a disjoint union. If $k_{r} \leq j<k_{r+1}$ we have that $K_{B_{j}}=\left\{k_{1}<\cdots<k_{r}\right\}$ and $L_{B_{j}}=\left\{l_{1}, \ldots, l_{r}\right\}$.

We have that $x_{k_{1}}, \ldots, x_{k_{d / 2}}, x_{l_{1}}, \ldots, x_{l_{d / 2}}$ is a basis in $V_{j}\left(\bmod V_{B_{j}}\right)$ for all $j \geq k_{d / 2}$. If $k_{r} \leq j<k_{r+1}, r<d / 2$, we have that $x_{k_{1}}, \ldots, x_{k_{r}}, x_{l_{1}}, \ldots, x_{l_{r}}$ is a basis in $V_{j}\left(\bmod \left(V_{j}\right)_{B_{j}}\right)$.

4.2. Coarse and fine $\mathscr{F}$-stratification of the dual of a nilpotent Lie algebra. Let $G$ be a connected, simply connected nilpotent Lie group with Lie algebra $\mathfrak{g}$, and let

$$
\mathscr{F}: \mathfrak{g}=\mathfrak{g}_{m} \supset \mathfrak{g}_{m-1} \supset \cdots \supset \mathfrak{g}_{1} \supset \mathfrak{g}_{0}=\{0\}
$$

be a fixed Jordan-Hölder sequence in $\mathfrak{g}$, and let $\mathscr{B}: X_{1}, \ldots, X_{m}$ be a compatible Jordan-Hölder basis in $\mathfrak{g}$, i.e. $X_{j} \in \mathfrak{g}_{j} \backslash \mathfrak{g}_{j-1}$.

For each $g \in \mathfrak{g}^{*}$ we have defined the skew-symmetric bilinear form $B_{g}: \mathfrak{g} \times$ $\mathfrak{g} \rightarrow \mathbf{R}$. We set $J_{g}$ to be the set of jump indices of $B_{g}$ relative to the flag $\mathscr{F}$, i.e. $J_{g}=J_{B_{g}}$ with the notation from $\S 4.1$. The restriction $\left(B_{g}\right)_{j}$ of $B_{g}$ to $\mathfrak{g}_{j} \times \mathfrak{g}_{j}$ is equal to $B_{g_{j}}$, where $g_{j}=g \mid \mathfrak{g}_{j}$. Therefore, if we set $J_{g}^{j}=J_{B_{g_{j}}}$ we have that the $m$-tuple of jump indices of $B_{g}$ is

$$
\mathscr{J}_{g}=\mathscr{J}_{B_{g}}=\left(J_{g}^{1}, J_{g}^{2}, \ldots, J_{g}^{m-1}, J_{g}^{m}\right) .
$$

Let us note that we have

$$
J_{g}^{j}=\left\{1 \leq k \leq j \mid X_{k} \notin \mathfrak{g}_{k-1}+\left(\mathfrak{g}_{j}\right)_{g_{j}}\right\} .
$$

We set $\mathscr{E}=\left\{J_{g} \mid g \in \mathfrak{g}^{*}\right\}$, and for $e \in \mathscr{E}$ we set $\Omega_{e}=\left\{g \in \mathfrak{g}^{*} \mid J_{g}=e\right\}$. Similarly we define $\mathscr{E}_{j}=\left\{J_{g}^{j} \mid g \in \mathfrak{g}^{*}\right\}$, and we set $\mathscr{E}=\left\{\mathscr{J}_{g} \mid g \in \mathfrak{g}^{*}\right\}$. Clearly $\mathscr{E} \subset \mathscr{E}_{1} \times \cdots \times \mathscr{E}_{m}$.

If $\varepsilon=\left(e_{1}, \ldots, e_{m}\right) \in \mathscr{E}$ we have that $e_{1} \subset e_{2} \subset \cdots \subset e_{m-1} \subset e_{m}$, and we define

$$
K_{\varepsilon}=\left\{1 \leq j \leq m \mid e_{j-1} \not \subset e_{j}\right\}=\left\{1 \leq j \leq m \mid j \in e_{j}\right\} .
$$

We write $K_{\varepsilon}=\left\{k_{1}<\cdots<k_{d / 2}\right\}$, and define the sequence $l_{1}, \ldots, l_{d / 2}$ recursively as follows:

$$
e_{k_{1}}=\left\{l_{1}<k_{1}\right\}, \quad e_{k_{r+1}}=e_{k_{r}} \cup\left\{l_{r+1}<k_{r+1}\right\}, \quad r<d / 2-1,
$$


and we set $L_{\varepsilon}=\left\{l_{1}, \ldots, l_{d / 2}\right\}$. Then $e_{m}=K_{\varepsilon} \cup L_{\varepsilon}$ as a disjoint union. We set $\varepsilon_{j}=\left(e_{1}, \ldots, e_{j}\right)$. If $k_{r} \leq j<k_{r+1}$ we have that $K_{\varepsilon_{j}}=\left\{k_{1}<\cdots<k_{r}\right\}$ and $L_{\varepsilon_{j}}=\left\{l_{1}, \ldots, l_{r}\right\}$, and $e_{j}=K_{\varepsilon_{j}} \cup L_{\varepsilon_{j}}$.

Let $e$ be a subset of $\{1, \ldots, m\}$. If $e=\varnothing$ we set $\mathbf{M}_{e}=1, Q_{e}=1$ and $P_{e}=1$. If $e \neq 0$ we write $e=\left\{j_{1}<\cdots<j_{d}\right\}$ and set for each $g \in \mathfrak{g}^{*} \mathbf{M}_{e}(g)$ to be the $d \times d$-matrix defined by

$$
\mathbf{M}_{e}(g)=\left[\left\langle g,\left[X_{j_{u}}, X_{j_{v}}\right]\right\rangle\right]_{1 \leq u, v \leq d},
$$

and set

$$
Q_{e}(g)=\operatorname{det} \mathbf{M}_{e}(g)
$$

and

For $\varepsilon \in \mathscr{E}$ we set

$$
P_{e}(g)=\operatorname{Pf} \mathbf{M}_{e}(g)
$$

$$
\Omega_{\varepsilon}=\left\{g \in \mathfrak{g}^{*} \mid \mathscr{J}_{g}=\varepsilon\right\} .
$$

We clearly have that $\mathfrak{g}^{*}=\bigcup_{\varepsilon \in \mathscr{E}} \Omega_{\varepsilon}$ as a finite, disjoint union. We call this partition of $\mathfrak{g}^{*}$ the fine $\mathscr{F}$-stratification of $\mathfrak{g}^{*}$ with respect to $\mathscr{F}$.

Similarly we call the partition $\mathfrak{g}^{*}=\bigcup_{e \in \mathscr{E}} \Omega_{e}$ into finitely many disjoint sets for the coarse $\mathscr{F}$-stratification of $\mathfrak{g}^{*}$ with respect to $\mathscr{F}$.

Let $\varepsilon \in \mathscr{E}$ and write $\varepsilon=\left(e_{1}, \ldots, e_{m}\right)$. We have

Lemma 4.2.1. The polynomial functions $P_{e_{j}}$ and $Q_{e_{j}}$ are constant on each coadjoint orbit $O$ contained in $\Omega_{\varepsilon}$.

Proof. If $O \subset \Omega_{\varepsilon}$ and if $g \in O$ then $J_{g}^{j}=e_{j}$. Let us write $e_{j}=\left\{j_{1}<\cdots<\right.$ $\left.j_{d}\right\}$. Since $\mathfrak{g}_{j}$ is an ideal we can write for each $s \in G$ and each $1 \leq v \leq d$ :

$$
\operatorname{Ad}\left(s^{-1}\right) X_{j_{v}}=\sum_{u=1}^{d} a_{u v} X_{j_{u}}+Z_{v},
$$

where $Z_{u} \in\left(\mathfrak{g}_{j}\right)_{g_{j}}$, and we have that $a_{u v}=0$ for $u>v$ and $a_{v v}=1$. If we let $\mathbf{A}$ be the matrix $\left[a_{u v}\right]_{1 \leq u, v \leq d}$ we have

$$
\begin{aligned}
\left\langle s g,\left[X_{j_{u}}, X_{j_{v}}\right]\right\rangle & =\left\langle g,\left[\operatorname{Ad}\left(s^{-1}\right) X_{j_{u}}, \operatorname{Ad}\left(s^{-1}\right) X_{j_{v}}\right]\right\rangle \\
& =\sum_{p, q=1}^{d} a_{p u}\left\langle g,\left[X_{j_{p}}, X_{j_{q}}\right]\right\rangle a_{q v}=\left({ }^{t} \mathbf{A M}_{e_{j}}(g) \mathbf{A}\right)_{u v},
\end{aligned}
$$

which means that $\mathbf{M}_{e_{j}}(s g)={ }^{t} \mathbf{A} \mathbf{M}_{e_{j}}(g) \mathbf{A}$. Now since $\mathbf{A}$ is an upper triangular matrix whose diagonal elements are all equal to 1 we have that $\operatorname{det} \mathbf{A}=1$, hence $P_{e_{j}}(s g)=\operatorname{Pf}_{e_{j}}(s g)=\operatorname{Pf}^{t} \mathbf{A} \mathbf{M}_{e_{j}}(g) \mathbf{A}=\operatorname{det} \mathbf{A P f} \mathbf{M}_{e_{j}}=\operatorname{Pf} \mathbf{M}_{e_{j}}=P_{e_{j}}(g)$. This ends the proof of the lemma, since $Q_{e_{j}}=P_{e_{j}}^{2}$.

Recall (see $\S 3.1$ ) that we have set

$$
\mathfrak{h}(g, \mathscr{F})=\sum_{j=1}^{m}\left(\mathfrak{g}_{j}\right)_{g_{\jmath}},
$$


and that $\mathfrak{h}(g, \mathscr{F})$ is a polarization at $g[3,1.2 .10]$, the Vergne polarization at $g$ associated with $\mathscr{F}$.

If $g \in \Omega_{\varepsilon}$ we also write $K_{g}=K_{\varepsilon}$ and $L_{g}=L_{\varepsilon}$. For $g \in \mathfrak{g}^{*}$ we can characterize $K_{g}$ as follows:

$$
K_{g}=\left\{1 \leq j \leq m \mid X_{j} \notin \mathfrak{g}_{j-1}+\mathfrak{h}(g, \mathscr{F})\right\}
$$

\section{CONSTRUCTION OF CANONICAL COORDINATES}

Let $G$ be a connected, simply connected nilpotent Lie group with Lie algebra $\mathfrak{g}$. In this section we shall show how one can explicitly construct global canonical coordinates on each coadjoint orbit of $G$. Actually, we do more than that: We give an algorithm for constructing the rational functions $p_{1}^{\varepsilon}, \ldots, p_{d / 2}^{\varepsilon}, q_{1}^{\varepsilon}, \ldots$, $q_{d / 2}^{\varepsilon}$ appearing in the theorem below. This theorem is the main result of this section. As a by-product we obtain non-inductive proofs of two basic facts from the Kirillov theory (5.4.6), namely that one can associate a well-defined class of irreducible representations of $G$ with each coadjoint orbit $O$ of $G$, and that the image of the universal enveloping algebra by the differential of such a representation can be identified with the algebra of all polynomial differential operators on some space $\mathbf{R}^{n}$ (the Weyl algebra).

5.1. Formulation of theorem. Let $\mathscr{F}$ be a Jordan-Hölder sequence in $\mathfrak{g}$, and let $\mathscr{B}: X_{1}, \ldots, X_{m}$ be a basis in $\mathfrak{g}$ compatible with $\mathscr{F}$. Recall that we have defined the fine $\mathscr{F}$-stratification $\mathfrak{g}^{*}=\bigcup_{\varepsilon \in \mathscr{G}} \Omega_{\varepsilon}$ of $\mathfrak{g}^{*}$, and that $F(O, \mathscr{F})$ is the $G$-invariant polarization of $O$ defined by the Vergne polarization $\mathfrak{h}(g)=$ $\mathfrak{h}(g, \mathscr{F})$ at $g \in \mathfrak{g}^{*}(\S 4.2)$.

Theorem 5.1.1. There exists for each $\varepsilon \in \mathscr{E}$ rational functions $p_{1}^{\varepsilon}, \ldots, p_{d / 2}^{\varepsilon}$, $q_{1}^{\varepsilon}, \ldots, q_{d / 2}^{\varepsilon}$ on $\mathfrak{g}^{*}$ with the following properties: For each orbit $O$ contained in $\Omega_{\varepsilon}$ the restrictions $q_{1}^{O}, \ldots, q_{d / 2}^{O}$ of $q_{1}^{\varepsilon}, \ldots, q_{d / 2}^{\varepsilon}$ to $O$ are in $\mathscr{P}_{F(O, \mathscr{F})}^{0}(O)$ and the restrictions $p_{1}^{O}, \ldots, p_{d / 2}^{O}$ of $p_{1}^{\varepsilon}, \ldots, p_{d / 2}^{\varepsilon}$ to $O$ are in $\mathscr{P}_{F(O, F)}^{1}(O)$, and $p_{1}^{O}, \ldots, p_{d / 2}^{O}, q_{1}^{O}, \ldots, q_{d / 2}^{O}$ are canonical coordinates on $O$.

We shall prove Theorem 5.1.1 in $\S \S 5.2,5.3,5.4$ and 5.5. In the course of our proof we shall describe an algorithm for calculating the the rational functions $p_{1}^{\varepsilon}, \ldots, p_{d / 2}^{\varepsilon}, q_{1}^{\varepsilon}, \ldots, q_{d / 2}^{\varepsilon}$ appearing in the theorem, and, in fact, this algorithm produces rational functions with denominators from the algebra generated by $P_{e_{1}}, \ldots, P_{e_{m}}$.

5.2. The rational functions $S_{j}^{\varepsilon}$; defining $q_{u}^{\varepsilon}$. The main goal of this section is to define the rational functions $q_{1}^{\varepsilon}, \ldots, q_{d / 2}^{\varepsilon}$ appearing in Theorem 5.1.1. 
For $g \in \Omega_{\varepsilon}$ we define the $(m \times d / 2)$-matrix $\boldsymbol{\tau}^{\varepsilon}(g)=\left[\tau_{j u}^{\varepsilon}(g)\right]_{\substack{1 \leq j \leq m \\ 1 \leq u \leq d / 2}}$ as follows:

$$
X_{j}=\sum_{u=1}^{d / 2} \tau_{j u}^{\varepsilon}(g) X_{k_{u}}+B_{j}^{\varepsilon}(g)
$$

where $B_{j}^{\varepsilon}(g) \in \mathfrak{h}(g)$. The matrix $\boldsymbol{\tau}^{\varepsilon}(g)$ is well defined since $X_{k_{1}}, \ldots, X_{k_{d / 2}}$ is a basis for $\mathfrak{g}(\bmod \mathfrak{h}(g))$ for $g \in \Omega_{\varepsilon}$.

Lemma 5.2.1. For all $g \in \Omega_{\varepsilon}$ and $h \in H=H(g)=\exp \mathfrak{h}(g)$ we have that $\boldsymbol{\tau}^{\varepsilon}(h g)=\boldsymbol{\tau}^{\varepsilon}(g)$ and $B_{j}(h g)=B_{j}(g), 1 \leq j \leq m$.

Proof. In general we have $\mathfrak{h}(s g)=\operatorname{Ad}(s) \mathfrak{h}(g)$, and since $\mathfrak{h}(g)$ is a subalgebra we have $\mathfrak{h}(h g)=\operatorname{Ad}(h) \mathfrak{h}(g)=\mathfrak{h}(g)$ for $h \in H(g)$. Therefore writing

$$
X_{j}=\sum_{u=1}^{d / 2} \tau_{j u}^{\varepsilon}(h g) X_{k_{u}}+B_{j}(h g),
$$

where $B_{j}(h g) \in \mathfrak{h}(h g)=\mathfrak{h}(g)$ we see that $B_{j}(h g)=B_{j}(g)$, and $\tau_{j u}^{\varepsilon}(h g)=$ $\tau_{j u}^{\varepsilon}(g)$ for all $g \in \Omega_{\varepsilon}, h \in H(g)$.

For $1 \leq j \leq m$ we define the function $S_{j}^{\varepsilon}$ on $\Omega_{\varepsilon}$ as follows

$$
S_{j}^{\varepsilon}(g)=\left\langle g, B_{j}^{\varepsilon}(g)\right\rangle
$$

we also have

$$
S_{j}^{\varepsilon}(g)=\left\langle g, X_{j}\right\rangle-\sum_{u=1}^{d / 2} \tau_{j u}^{\varepsilon}(g)\left\langle g, X_{k_{u}}\right\rangle
$$

Lemma 5.2.2. For $g \in \Omega_{\varepsilon}$ and $h \in H=H(g)=\exp \mathfrak{h}(g)$ we have $S_{j}^{\varepsilon}(h g)=$ $S_{j}^{\varepsilon}(g)$.

Proof. For $h \in H(g)$ we have $S_{j}^{\varepsilon}(h g)=\left\langle h g, B_{j}(h g)\right\rangle=\left\langle h g, B_{j}(g)\right\rangle$ Now $h=\exp X, X \in \mathfrak{h}(g)$, hence $\left\langle h g, B_{j}(g)\right\rangle=\left\langle g, e^{-\operatorname{ad} X} B_{j}(g)\right\rangle=\left\langle g, B_{j}(g)\right\rangle$, since $\mathfrak{h}(g)$ is an isotropic subalgebra.

It follows from Lemma 5.2.2 that $S_{j}^{\varepsilon}$ when restricted to an orbit $O=G g$ contained in $\Omega_{\varepsilon}$ is constant on the fibers of the fibration defined by $H=H(g)$. The definition of $q_{1}^{\varepsilon}, \ldots, q_{d / 2}^{\varepsilon}$ on $\Omega_{\varepsilon}$ now are as follows: Write $L_{g}=\left\{\bar{l}_{1}<\right.$ $\left.\cdots<\bar{l}_{d / 2}\right\}$ and set $q_{u}^{\varepsilon}(g)=S_{\bar{l}_{u}}^{\varepsilon}(g)$ for $g \in \Omega_{\varepsilon}, u=1, \ldots, d / 2$. In order to be able to extend $q_{1}^{\varepsilon}, \ldots, q_{d / 2}^{\varepsilon}$ to all of $\mathfrak{g}^{*}$ as rational functions and to give an algorithm for computing such extensions, we shall have to explore the matrix $\boldsymbol{\tau}^{\varepsilon}$ in greater detail.

5.3. The matrix $\tau$. Let $\varepsilon \in \mathscr{E}$, and let $d$ be the dimension of orbits contained in $\Omega_{\varepsilon}$. 
For each $\mathfrak{g} \in \Omega_{\varepsilon}$ we define the $(m \times d)$-matrix $\rho^{d / 2}(g)$ as follows: For each $1 \leq j \leq m$ we write

$$
X_{j}=\sum_{u=1}^{d / 2} \rho_{j 2 u-1}^{d / 2}(g) X_{k_{u}}+\sum_{u=1}^{d / 2} \rho_{j 2 u}^{d / 2} X_{l_{u}}+A_{j}^{d / 2}(g)
$$

where $A_{j}^{d / 2}(g) \in \mathfrak{g}_{g}$.

For each $1 \leq v \leq d / 2$ we then have

(\#) $\left\langle g,\left[X_{j}, X_{k_{v}}\right]\right\rangle=\sum_{u=1}^{d / 2} \rho_{j 2 u-1}^{d / 2}(g)\left\langle g,\left[X_{k_{u}}, X_{k_{v}}\right]\right\rangle+\sum_{u=1}^{d / 2} \rho_{j 2 u}^{d / 2}(g)\left\langle g,\left[X_{l_{u}}, X_{k_{v}}\right]\right\rangle$,

$$
\left\langle g,\left[X_{j}, X_{l_{v}}\right]\right\rangle=\sum_{u=1}^{d / 2} \rho_{j 2 u-1}^{d / 2}(g)\left\langle g,\left[X_{k_{u}}, X_{l_{v}}\right]\right\rangle+\sum_{u=1}^{d / 2} \rho_{j 2 u}^{d / 2}(g)\left\langle g,\left[X_{l_{u}}, X_{l_{v}}\right]\right\rangle .
$$

In order to rewrite the relations $(\sharp)$ in a more compact form we introduce for any $g \in \mathfrak{g}^{*}$ the $(d \times d)$-matrix $\boldsymbol{\mu}^{d / 2}$ and the $(m \times d / 2)$-matrix $\boldsymbol{\sigma}^{d / 2}(g)$ as follows

$$
\begin{array}{cc}
\sigma_{j 2 u-1}^{d / 2}=\left\langle g,\left[X_{j}, X_{k_{u}}\right]\right\rangle, & 1 \leq j \leq m, 1 \leq u \leq d / 2, \\
\sigma_{j 2 u}^{d / 2}=\left\langle g,\left[X_{j}, X_{l_{u}}\right]\right\rangle, & 1 \leq j \leq m, 1 \leq u \leq d / 2
\end{array}
$$

and

$$
\begin{array}{cc}
\mu_{2 u-12 v-1}^{d / 2}(g)=\left\langle g,\left[X_{k_{u}}, X_{k_{v}}\right]\right\rangle, & 1 \leq u, v \leq d / 2, \\
\mu_{2 u-12 v}^{d / 2}(g)=\left\langle g,\left[X_{k_{u}}, X_{l_{v}}\right]\right\rangle, & 1 \leq u, v \leq d / 2, \\
\mu_{2 u 2 v-1}^{d / 2}(g)=\left\langle g,\left[X_{l_{u}}, X_{k_{v}}\right]\right\rangle, & 1 \leq u, v \leq d / 2, \\
\mu_{2 u 2 v}^{d / 2}(g)=\left\langle g,\left[X_{l_{u}}, X_{l_{v}}\right]\right\rangle, & 1 \leq u, v \leq d / 2 .
\end{array}
$$

The relations $(\sharp)$ can then be expressed as

$$
\boldsymbol{\sigma}^{d / 2}(g)=\boldsymbol{\rho}^{d / 2}(g) \boldsymbol{\mu}^{d / 2}(g),
$$

for $g \in \Omega_{\varepsilon}$, hence we have for $g \in \Omega_{\varepsilon}$ :

$$
\boldsymbol{\rho}^{d / 2}(g)=\boldsymbol{\sigma}^{d / 2}(g) \boldsymbol{\mu}^{d / 2}(g)^{-1} \text {. }
$$

We shall take this formula as the definition of $\rho^{d / 2}(g)$ when $g$ does not belong to $\Omega_{\varepsilon}$. In this way the entries of the matrix $\rho^{d / 2}(g)$ are rational functions on $\mathfrak{g}^{*}$ with denominators equal to $P_{d / 2}(g)=\operatorname{Pf} \mu^{d / 2}(g)$.

Similarly we define for $1 \leq r<d / 2$ the $\left(k_{r+1}-1\right) \times 2 r$-matrix $\rho^{r}(g)$ as follows: For each $1 \leq j \leq\left(k_{r+1}-1\right)$ we write

$$
X_{j}=\sum_{u=1}^{r} \rho_{j 2 u-1}^{r}(g) X_{k_{u}}+\sum_{u=1}^{r} \rho_{j 2 u}^{r}(g) X_{l_{u}}+A_{j}^{r}(g),
$$

where $A_{j}^{r}(g) \in\left(\mathfrak{g}_{k}\right)_{g_{k}}$, where $k=k_{r+1}-1$. 
We also define the $\left(k_{r+1}-1\right) \times 2 r$-matrix $\boldsymbol{\sigma}^{r}(g)$ and the $(2 r \times 2 r)$-matrix $\boldsymbol{\mu}^{r}(g)$ as follows

$$
\begin{aligned}
& \sigma_{j 2 u-1}^{r}(g)=\left\langle g,\left[X_{j}, X_{k_{u}}\right]\right\rangle, \quad 1 \leq j<k_{r+1}, 1 \leq u \leq r, \\
& \sigma_{j 2 u}^{r}(g)=\left\langle g,\left[X_{j}, X_{l_{u}}\right]\right\rangle, \quad 1 \leq j<k_{r+1}, 1 \leq u \leq r,
\end{aligned}
$$

and

$$
\begin{aligned}
\mu_{2 u-12 v-1}^{r}(g)=\left\langle g,\left[X_{k_{u}}, X_{k_{v}}\right]\right\rangle, & 1 \leq u, v \leq r, \\
\mu_{2 u-12 v}^{r}(g)=\left\langle g,\left[X_{k_{u}}, X_{l_{v}}\right]\right\rangle, & 1 \leq u, v \leq r, \\
\mu_{2 u 2 v-1}^{r}(g)=\left\langle g,\left[X_{l_{u}}, X_{k_{v}}\right]\right\rangle, & 1 \leq u, v \leq r, \\
\mu_{2 u 2 v}^{r}(g)=\left\langle g,\left[X_{l_{u}}, X_{l_{v}}\right]\right\rangle, & 1 \leq u, v \leq r,
\end{aligned}
$$

and in this way we have as above

$$
\boldsymbol{\sigma}^{r}(g)=\boldsymbol{\rho}^{r}(g) \boldsymbol{\mu}^{r}(g)
$$

and

$$
\boldsymbol{\rho}^{r}(g)=\boldsymbol{\sigma}^{r}(g) \boldsymbol{\mu}^{r}(g)^{-1}
$$

for $g \in \Omega_{\varepsilon}$. We take the last relation as the definition of $\boldsymbol{\rho}^{r}(g)$ when $g$ is not contained in $\Omega_{\varepsilon}$. In this way the entries of $\boldsymbol{\rho}^{r}(g)$ are rational functions in $g$ with denominators in the algebra generated by the polynomials by $P_{r}(g)=$ $\operatorname{Pf} \boldsymbol{\mu}^{r}(g), 1 \leq r \leq d / 2$. Note that the matrices $\boldsymbol{\sigma}^{r}$ and $\boldsymbol{\mu}^{r}, r=1, \ldots, d / 2$, are submatrices of $\boldsymbol{\sigma}^{d / 2}$ and $\boldsymbol{\mu}^{d / 2}$, respectively.

We note that $\boldsymbol{\rho}^{r}(g)$ depends only on $g \mid \mathfrak{g}_{k_{r}}$. We write this symbolically as $\boldsymbol{\rho}^{r}(g)=\boldsymbol{\rho}^{r}\left(\xi_{1}, \ldots, \xi_{k_{r}}\right)$, where $\left(\xi_{1}, \ldots, \xi_{m}\right)$ are the coordinates of $g$ with respect to the basis of $\mathfrak{g}^{*}$ dual to $X_{1}, \ldots, X_{m}$.

For $g \in \Omega_{\varepsilon}$ we have defined the $(m \times d / 2)$-matrix $\boldsymbol{\tau}(g)=\left[\tau_{j u}^{\varepsilon}\right]_{\substack{1 \leq j \leq m \\ 1 \leq u \leq d / 2}}$ as follows:

$$
X_{j}=\sum_{u=1}^{d / 2} \tau_{j u}^{\varepsilon}(g) X_{k_{u}}+B_{j}(g),
$$

where $B_{j}(g) \in \mathfrak{h}(g)$.

We shall give a recursive way of obtaining $\tau_{j u}^{\varepsilon}$ :

If $j<k_{1}$ we have that $\tau_{j u}^{\varepsilon}(g)=0$ for $1 \leq u \leq d / 2$.

Suppose then that $\tau_{j u}^{\varepsilon}(g)$ has been found for all $j<k_{r}, 1 \leq u \leq d / 2$. If $r<d$ we shall determine $\tau_{j u}^{\varepsilon}$ for all $k_{r} \leq j<k_{r+1}, 1 \leq u \leq d / 2$, and if $r=d$ we shall determine $\tau_{j u}^{\varepsilon}$ for all $k_{d / 2} \leq j \leq m, 1 \leq u \leq d / 2$. So suppose $k_{r} \leq j<k_{r+1}$ (resp. $k_{d} \leq j \leq m$ ). We can then write

$$
X_{j}=\sum_{u=1}^{r} \rho_{j 2 u-1}^{r}(g) X_{k_{u}}+\sum_{u=1}^{r} \rho_{j 2 u}^{r}(g) X_{l_{u}}+A_{j}^{r}(g)
$$


We also write

$$
X_{l_{u}}=\sum_{v=1}^{d / 2} \tau_{l_{u} v}(g) X_{k_{v}}+B_{l_{u}}(g)
$$

$u=1, \ldots, d$.

Now if $u \leq r$, then $l_{u}<k_{u}<k_{r}$, so

$$
X_{l_{u}}=\sum_{v=1}^{r} \tau_{l_{u} v}^{\varepsilon}(g) X_{k_{v}}+B_{l_{u}}(g)
$$

$u=1, \ldots, r$, hence

$$
\begin{aligned}
X_{j}= & \sum_{u=1}^{r} \rho_{j 2 u-1}^{r}(g) X_{k_{u}}+\sum_{u=1}^{r} \rho_{j 2 u}^{r}(g)\left(\sum_{v=1}^{r} \tau_{l_{u} v}^{\varepsilon}(g) X_{k_{v}}+B_{l_{u}}^{\varepsilon}(g)\right)+A_{j}^{r}(g) \\
= & \sum_{u=1}^{r} \rho_{j 2 u-1}^{r}(g) X_{k_{u}}+\sum_{u=1}^{r} \sum_{v=1}^{r} \rho_{j 2 u}^{r}(g) \tau_{l_{u} v}^{\varepsilon}(g) X_{k_{v}} \\
& +\sum_{u=1}^{r} \rho_{j 2 u}^{r}(g) B_{l_{u}}^{\varepsilon}(g)+A_{j}^{r}(g) \\
= & \sum_{u=1}^{r}\left(\rho_{j 2 u-1}^{r}(g)+\sum_{v=1}^{r} \rho_{j 2 v}^{r}(g) \tau_{l_{v} u}^{\varepsilon}(g)\right) X_{k_{u}} \\
& +\sum_{u=1}^{r} \rho_{j 2 u}^{r}(g) B_{l_{u}}^{\varepsilon}(g)+A_{j}^{r}(g) .
\end{aligned}
$$

Now since the last term is clearly contained in $\mathfrak{h}(g)$ we have for $k_{r} \leq j<k_{r+1}$ $\left(k_{d} \leq j \leq m\right)$ :

$$
\begin{aligned}
& \tau_{j u}^{\varepsilon}(g)=\rho_{j 2 u-1}^{r}(g)+\sum_{v=1}^{r} \rho_{j 2 v}^{r}(g) \tau_{l_{v} u}^{\varepsilon}(g), \quad 1 \leq u \leq r, \\
& \tau_{j u}^{\varepsilon}(g)=0, \quad r<u \leq d / 2 .
\end{aligned}
$$

We take this as the defining relations for $\tau^{\varepsilon}(g)$ when $g$ does not belong to $\Omega_{\varepsilon}$. In this way $\tau_{j u}^{\varepsilon}$ is a rational function on $\mathfrak{g}^{*}$ with denominators from the algebra genarated by the polynomials $P_{1}, \ldots, P_{d / 2}$. We see that $\tau_{j u}^{\varepsilon}(g)=$ $\tau_{j u}^{\varepsilon}\left(\xi_{1}, \ldots, \xi_{k_{r}}\right)$ for $k_{r} \leq j<k_{r+1}$.

Lemma 5.3.1. The functions $\tau_{j u}^{\varepsilon}$, when restricted to a single orbit $O$ contained in $\Omega_{\varepsilon}$, belong to $\mathscr{P}_{F(O, \mathscr{F})}^{0}(O)$.

Proof. We already know that $\tau_{j u}^{\varepsilon}$ are rational functions with denominators in the algebra generated by $P_{1}, \ldots, P_{d / 2}$ and that $\tau_{j u}^{\varepsilon}(h g)=\tau_{j u}^{\varepsilon}(g)$ for $g \in \Omega_{\varepsilon}$, $h \in H(g)$ (Lemma 5.2.1). Now since $P_{1}, \ldots, P_{d / 2}$ are constant when restricted to a single orbit contained in $\Omega_{\varepsilon}$ (Lemma 4.2.1) we have proved the lemma. 
We have defined the functions $S_{j}^{\varepsilon}$ on $\Omega_{\varepsilon}$ by

$$
S_{j}^{\varepsilon}(g)=\left\langle g, X_{j}\right\rangle-\sum_{u=1}^{d / 2} \tau_{j u}^{\varepsilon}(g)\left\langle g, X_{k_{u}}\right\rangle .
$$

We take this as the defining relation of $S_{j}^{\varepsilon}$ when $g$ does not belong to $\Omega_{\varepsilon}$. In this way $S_{j}^{\varepsilon}$ is a rational function on $\mathfrak{g}^{*}$. For $j=k_{r}, r=1, \ldots, d$, we have $S_{j}^{\varepsilon}=0$, and for $k_{r}<j<k_{r+1}$ we have

$$
S_{j}^{\varepsilon}(g)=\xi_{j}+\bar{S}_{j}^{\varepsilon}\left(\xi_{1}, \ldots, \xi_{k_{r}}\right)
$$

where $\bar{S}_{j}^{\varepsilon}(g)$ is a rational function on $\mathfrak{g}^{*}$ with denominators in the algebra generated by $P_{1}, \ldots, P_{r}$. It follows that we have, using Lemma 5.2.2,

Lemma 5.3.2. The functions $S_{j}^{\varepsilon}, 1 \leq j \leq m$, when restricted to a single orbit $O$ contained in $\Omega_{\varepsilon}$, belong to $\mathscr{P}_{F(O, \mathscr{F})}^{0}(O)$.

5.4. Construction of canonical coordinates on a single orbit. We now consider the $\mathscr{B}$-chart $\sigma_{O}^{\mathscr{B}}: O \rightarrow \mathbf{R}^{d}$ of a single orbit $O=G g$ contained in $\Omega_{\varepsilon}$. Recall that $\sigma_{O}^{\mathscr{B}}$ is defined as $\sigma_{O}^{\mathscr{B}}: l \rightarrow\left(\left\langle l, X_{j_{1}}\right\rangle, \ldots,\left\langle l, X_{j_{d}}\right\rangle\right)$ (see $\left.\S 1.6\right)$. In the following we use then notation $\sigma_{O}^{\mathscr{B}}=\left(y_{j_{1}}, \ldots, y_{j_{d}}\right)$. The inverse of $\sigma_{O}^{\mathscr{B}}$ is given by $\sigma_{O}^{\mathscr{B}}-1:\left(y_{j_{1}}, \ldots, y_{j_{d}}\right)=y \rightarrow\left(R_{1}(g, y), \ldots, R_{m}(g, y)\right)=\sum_{j=1}^{m} R_{j}(g, y) l_{j}$. Recall that $(g, y) \rightarrow R_{j}(g, y)$ is a polynomial function in the variable $y=$ $\left(y_{j_{1}}, \ldots, y_{j_{d}}\right)$ and a rational function in the variable $g \in \mathfrak{g}^{*}$ with denominators in the algebra genarated by $P_{1}, \ldots, P_{d / 2}$, and that $R_{j_{r}}(g, y)=y_{j_{r}}$.

We now define another map $\tau_{O}^{\mathscr{B}}: O \rightarrow \mathbf{R}^{d}$ in the following manner: $\tau_{O}^{\mathscr{B}}:$ $l \rightarrow\left(z_{j_{1}}(l), \ldots, z_{j_{d}}(l)\right)$, where, for $r=1, \ldots, d / 2$,

$$
z_{j_{r}}(l)=\left\langle l, X_{j_{r}}\right\rangle \quad \text { if } j_{r} \in K_{g}
$$

and

$$
z_{j_{r}}(l)=S_{j_{r}}(l) \text { if } j_{r} \in L_{g}
$$

or, put otherwise,

$$
z_{k_{r}}(l)=\left\langle l, X_{k_{r}}\right\rangle=y_{k_{r}}(l)
$$

and

$$
z_{l_{r}}(l)=S_{l_{r}}^{\varepsilon}(l) \quad\left(\text { or } z_{\bar{l}_{r}}(l)=S_{l_{r}}^{\varepsilon}(l)\right) .
$$

Then $z_{j_{1}}, \ldots, z_{j_{d}}$ are polynomial functions on the orbit $O$, and they belong to $\mathscr{P}_{F(O, \mathscr{F})}^{1}(O)$, and for $j_{r} \in L_{g}$ we have that $z_{j_{r}} \in \mathscr{P}_{F}^{0}(O)$ (Lemma 5.2.2).

Lemma 5.4.1. The map $\tau_{O}^{\mathscr{B}}$ is a polynomial chart on $O$. 
Proof. First we consider $\tau_{O}^{\mathscr{B}} \circ \sigma_{O}^{\mathscr{B}}-1: \mathbf{R}^{d} \rightarrow \mathbf{R}^{d}$. We write $\tau_{O}^{\mathscr{B}} \circ \sigma_{O}^{\mathscr{B}}-1(y)=$ $\left(f_{j_{1}}(g, y), \ldots, f_{j_{d}}(g, y)\right)$, where $O=G g$. We have for $1 \leq r \leq d / 2$

$$
\begin{aligned}
f_{k_{r}}(g, y) & =y_{k_{r}}, \\
f_{l_{r}}(g, y) & =S_{l_{r}}\left(R_{1}(g, y), \ldots, R_{m}(g, y)\right) \\
& =y_{l_{r}}+\bar{S}_{l_{r}}^{\varepsilon}\left(R_{1}(g, y), \ldots, R_{l_{r}-1}(g, y)\right) .
\end{aligned}
$$

We set $\bar{f}_{j_{r}}(g, y)=\bar{S}_{j_{r}}^{\varepsilon}\left(R_{1}(g, y), \ldots, R_{j_{r}-1}(g, y)\right)$ for $j_{r} \in L_{g}, 1 \leq r \leq d$. Since $R_{j}(g, y)$ at most depends on $y_{j_{1}}, \ldots, y_{j_{r-1}}$ for $j<j_{r}$ we have that

$$
\bar{f}_{j_{r}}(g, y)=\bar{f}_{j_{r}}\left(g, y_{1}, \ldots, y_{j_{r-1}}\right) \text {, }
$$

when $j_{r} \in L_{g}$. Let us set $\bar{f}_{j_{r}}=0$ for $j_{r} \in K_{g}, 1 \leq r \leq d$. In this way we have for all $1 \leq r \leq d$ :

$$
f_{j_{r}}(g, y)=y_{j_{r}}+\bar{f}_{j_{r}}(g, y)=y_{j_{r}}+\bar{f}_{j_{r}}\left(g, y_{j_{1}}, \ldots, y_{j_{r-1}}\right) .
$$

Writing these equations

$$
\begin{aligned}
z_{j_{1}} & =y_{j_{1}}, \\
z_{j_{2}} & =y_{j_{2}}+\bar{f}_{j_{2}}\left(g, y_{j_{1}}\right), \\
z_{j_{3}} & =y_{j_{3}}+\bar{f}_{j_{3}}\left(g, y_{j_{1}}, y_{j_{2}}\right), \\
& \ldots \\
z_{j_{d}} & =y_{j_{d}}+\bar{f}_{j_{d}}\left(g, y_{j_{1}}, \ldots, y_{j_{d-1}}\right),
\end{aligned}
$$

we see by succesive solution that

$$
\begin{aligned}
y_{j_{1}} & =z_{j_{1}}, \\
y_{j_{2}} & =z_{j_{2}}-\bar{f}_{j_{2}}\left(g, y_{j_{1}}\right)=z_{j_{2}}-\bar{f}_{j_{2}}\left(g, z_{1}\right), \\
y_{j_{3}} & =z_{j_{3}}-\bar{f}_{j_{3}}\left(g, y_{j_{1}}, y_{j_{2}}\right) \\
& =z_{j_{3}}-\bar{f}_{j_{3}}\left(g, z_{j_{1}}, z_{j_{2}}-\bar{f}_{j_{2}}\left(g, z_{j_{1}}\right)\right), \\
& \ldots \\
y_{j_{d}} & =z_{j_{d}}-\bar{f}_{j_{d}}\left(g, y_{j_{1}}, \ldots, y_{j_{d-1}}\right),
\end{aligned}
$$

and therefore that $\tau_{O}^{\mathscr{B}} \circ \sigma_{O}^{\mathscr{B}}-1$ is bijective and its inverse $z \rightarrow \sigma_{O}^{\mathscr{B}} \circ \tau_{O}^{\mathscr{B}}-1(z)=$ $\left(h_{j_{1}}(g, z), \ldots, h_{j_{d}}(g, z)\right): \mathbf{R}^{d} \rightarrow \mathbf{R}^{d}$ is a polynomial function given by

$$
y_{j_{r}}=h_{j_{r}}(g, z)=z_{j_{r}}+\bar{h}_{j_{r}}(g, z)=z_{j_{r}}+\bar{h}_{j_{r}}\left(g, z_{j_{1}}, \ldots, z_{j_{r-1}}\right) \text {, }
$$

$r=1, \ldots, d$. In other words, $\tau_{O}^{\mathscr{B}} \circ \sigma_{O}^{\mathscr{B}^{-1}}$ is a polynomial diffeomorphism. This ends the proof of the lemma.

5.4.2. For later use we note that the functions $(g, y) \rightarrow f_{j_{r}}(g, y)$ and $(g, z) \rightarrow$ $h_{j_{r}}(g, z), g \in \Omega_{\varepsilon}, y \in \mathbf{R}^{d}$, in a natural way can be extended to be rational 
functions on $\mathfrak{g}^{*}$ in the variable $g$ with denominators in the algebra generated by the polynomials $P_{1}, \ldots, P_{d / 2}$.

We shall now briefly consider what happens to the canonical symplectic form under the coordinate change $\left(y_{j_{1}}, \ldots, y_{j_{d}}\right) \rightarrow\left(z_{j_{1}}, \ldots, z_{j_{d}}\right)$. Let us set $\omega_{r s}=$ $\omega_{O}\left(\partial / \partial y_{j_{r}}, \partial / \partial y_{j_{s}}\right)$ and $\omega_{r s}^{\prime}=\omega_{O}\left(\partial / \partial z_{j_{r}}, \partial / \partial z_{j_{s}}\right)$ for $1 \leq r, s \leq d$, and let $\boldsymbol{\omega}, \boldsymbol{\omega}^{\prime}$ be the corresponding matrices. Writing $\boldsymbol{\gamma}=\left[\partial z_{j_{r}} / \partial y_{j_{s}}\right]_{1 \leq r, s \leq d}$ we have $\boldsymbol{\omega}={ }^{t} \boldsymbol{\gamma} \boldsymbol{\omega}^{\prime} \boldsymbol{\gamma}$. Now since $z_{j_{r}}=f_{j_{r}}(y)=y_{j_{r}}+\bar{f}_{j_{r}}\left(y_{j_{1}}, \ldots, y_{j_{r-1}}\right)$ we see that $\partial z_{j_{r}} / \partial y_{j_{s}}=1$ for $s=r$, and $\partial z_{j_{r}} / \partial y_{j_{s}}=0$ for $s>r$, and therefore that $\boldsymbol{\gamma}$ is a lower triangular matrix with 1 's in the diagonal, hence $\operatorname{det} \boldsymbol{\gamma}=1$. We conclude that $\operatorname{det} \boldsymbol{\omega}=\operatorname{det} \boldsymbol{\omega}^{\prime}$. Now we have the following matrix relations $\left[\left\{y_{j_{r}}, y_{j_{s}}\right\}\right]_{1 \leq r, s \leq d}=-\boldsymbol{\omega}^{-1}$, and similarly $\left[\left\{z_{j_{r}}, z_{j_{s}}\right\}\right]_{1 \leq r, s \leq d}=-\boldsymbol{\omega}^{\prime-1}$. It follows that

$$
\begin{aligned}
P_{e_{m}}(l)^{2} & =\operatorname{Pf}\left(\left[\left\langle l,\left[X_{j_{r}}, X_{j_{s}}\right]\right\rangle\right]_{1 \leq r, s \leq d}\right)^{2} \\
& =\operatorname{Pf}\left(\left[\left\{y_{j_{r}}, y_{j_{s}}\right\}(l)\right]_{1 \leq r, s \leq d}\right)^{2}=\operatorname{Pf}\left(\left[\left\{z_{j_{r}}, z_{j_{s}}\right\}(l)\right]_{1 \leq r, s \leq d}\right)^{2} .
\end{aligned}
$$

We now define the $(d \times d)$-matrix $\mathbf{A}$ in the following way:

$$
\begin{aligned}
& a_{r s}(l)=\left\{z_{k_{r}}, z_{k_{s}}\right\}, \quad 1 \leq r, s \leq d / 2, \\
& a_{r s+d / 2}(l)=\left\{z_{k_{r}}, z_{\bar{l}_{s}}\right\}, \quad 1 \leq r, s \leq d / 2 \text {, } \\
& a_{r+d / 2 s}(l)=\left\{z_{\bar{l}_{r}}, z_{k_{s}}\right\}, \quad 1 \leq r, s \leq d / 2, \\
& a_{r+d / 2 s+d / 2}(l)=\left\{z_{\bar{l}_{r}}, z_{\bar{l}_{s}}\right\}, \quad 1 \leq r, s \leq d / 2 \text {. }
\end{aligned}
$$

Let us then note that by construction $\left\{z_{\bar{l}_{r}}, z_{\bar{l}_{s}}\right\}=0$, hence $a_{r s}=0$ for $d / 2<r, s \leq d$, i.e., $\mathbf{A}(l)$ has the form

$$
\mathbf{A}(l)=\left(\begin{array}{cc}
\mathbf{A}_{11}(l) & \mathbf{A}_{12}(l) \\
-\mathbf{A}_{12}(l) & \mathbf{O}
\end{array}\right)
$$

It follows that $\operatorname{det} \mathbf{A}(l)=\left(\operatorname{det} \mathbf{A}_{12}(l)\right)^{2}$. But

$$
\operatorname{det} \mathbf{A}(l)=\operatorname{det}\left(\left[\left\{z_{j_{r}}, z_{j_{s}}\right\}\right]_{1 \leq r, s \leq d}(l)\right)=P_{e_{m}}(l)^{2},
$$

hence $\operatorname{det} \mathbf{A}_{12}(l)= \pm P_{e_{m}}(l)$. In particular $\operatorname{det} \mathbf{A}_{12}(l)$ is constant on each orbit contained in $\Omega_{\varepsilon}$. We define for each $l \in O$ the $(d / 2 \times d / 2)$-matrix $\mathbf{B}(\ell)$ as

$$
\mathbf{B}(l)=\mathbf{A}_{12}(l)^{-1}
$$

Since the entries of $\mathbf{A}_{12}$ are of the form $\left\{z_{k_{r}}, z_{l_{s}}\right\}$, and since $z_{l_{s}} \in \mathscr{P}_{F(O, \mathscr{F})}^{0}(O)$, we see that the entries of $\mathbf{A}_{12}$ are in $\mathscr{P}_{F(O, \mathscr{F})}^{0}(O)$. Moreover, since $\mathscr{P}_{F(O, \mathscr{F})}^{0}(O)$ is a subalgebra and since $\operatorname{det} \mathbf{A}_{12}$ is constant on $O$ we see that the entries of $\mathbf{B}$ are in $\mathscr{P}_{F(O, \mathscr{F})}^{0}(O)$. 
We now define the functions $q_{1}=q_{1}^{O}, \ldots, q_{d / 2}=q_{d / 2}^{O}$ and $\bar{p}_{1}=\bar{p}_{1}^{O}, \ldots$, $\bar{p}_{d / 2}=\bar{p}_{d / 2}^{O}$ as follows: We set

$$
\begin{aligned}
q_{r}(l) & =z_{\bar{l}_{r}}(l), \quad r=1, \ldots, d / 2, \\
\bar{p}_{r}(l) & =\sum_{s=1}^{d / 2} b_{r s}(l) z_{k_{s}}(l), \quad r=1, \ldots, d / 2 .
\end{aligned}
$$

We note that $q_{r}^{O}=q_{r}^{\varepsilon} \mid O, r=1, \ldots, d / 2$ (see $\left.\S 5.2\right)$. By construction we have $\left\{q_{r}, q_{s}\right\}=0$. Moreover, $\left\{\bar{p}_{r}, q_{s}\right\}=\left\{\sum_{t=1}^{d / 2} b_{r t} z_{k_{t}}, z_{\bar{l}_{s}}\right\}=\sum_{t=1}^{d / 2} b_{r t}\left\{z_{k_{t}}, z_{\bar{l}_{s}}\right\}=$ $\left(\mathbf{B A}_{12}\right)_{r s}=\delta_{r s}$. We also note that $q_{1}, \ldots, q_{d / 2} \in \mathscr{P}_{F}^{0}(O)$ and that $\bar{p}_{1}, \ldots, \bar{p}_{d / 2}$ $\in \mathscr{P}_{F}^{1}(O)$.

Define the map $\tau=\left(x_{1}, \ldots, x_{d / 2}\right): G / H \rightarrow \mathbf{R}^{d / 2}$ by $x_{r}(s H)=q_{r}(s g)$, $s \in G, 1 \leq r \leq d / 2$. This map is well defined by since $q_{r} \in \mathscr{P}_{F}^{0}(O)$.

Lemma 5.4.3. The map $\tau$ defines a polynomial chart on $G / H$.

Proof. It follows from Lemma 2.2 .6 that the map $\Phi: O \rightarrow \mathbf{R}^{d / 2} \times G / H$ given by

$$
\Phi: l=s g \rightarrow\left(\left\langle l, X_{k_{1}}\right\rangle, \ldots,\left\langle l, X_{k_{d / 2}}\right\rangle, s H\right)
$$

is a bi-polynomial diffeomorphism, and it follows from Lemma 5.4.1 that the map $O \rightarrow \mathbf{R}^{d}$ given by

$$
l \rightarrow\left(\left\langle l, X_{k_{1}}\right\rangle, \ldots,\left\langle l, X_{k_{d / 2}}\right\rangle, q_{1}(l), \ldots, q_{d / 2}\right)
$$

is a bi-polynomial diffeomorphism. But from this we see that the map $G / H \rightarrow$ $\mathbf{R}^{d / 2}: s H \rightarrow\left(q_{1}(s g), \ldots, q_{d / 2}(s g)\right)$ is a bi-polynomial diffeomorphism. This ends the proof of the lemma.

It follows from the previous lemma that $\mathscr{P}_{F}^{0}(O)$ are all functions of the form $a\left(q_{1}, \ldots, q_{d / 2}\right)$, where $a \in \mathscr{P}\left(\mathbf{R}^{d / 2}\right)$.

We now define the map $\bar{\varphi}_{O}^{\mathscr{B}}: O \rightarrow \mathbf{R}^{d}$ by

$$
\bar{\varphi}_{O}^{\mathscr{B}}: l \rightarrow\left(\bar{p}_{1}(l), \ldots, \bar{p}_{d / 2}(l), q_{1}(l), \ldots, q_{d / 2}(l)\right) .
$$

Lemma 5.4.4. $\bar{\varphi}_{O}^{\mathscr{B}}$ is a polynomial chart.

Proof. Consider $\bar{\varphi}_{O}^{\mathscr{B}} \circ \tau_{O}^{\mathscr{B}}-1$ : $z \rightarrow(\bar{p}(z), q(z))$. We have for $1 \leq r \leq d / 2$ :

$$
q_{r}(z)=z_{\bar{l}_{r}}, \quad \bar{p}_{r}=\sum_{s=1}^{d / 2} b_{r s}\left(z_{\bar{l}_{1}}, \ldots, z_{l_{d / 2}}\right) z_{k_{s}},
$$

so $\bar{\varphi}_{O}^{\mathscr{B}} \circ \tau_{O}^{\mathscr{B}-1}$ is a polynomial function. Moreover, for $1 \leq r \leq d / 2$ we have

$$
\begin{aligned}
& z_{l_{r}}=q_{r} \\
& z_{k_{r}}=\sum_{s=1}^{d / 2} a_{r s}(l) \bar{p}_{s}=\sum_{s=1}^{d / 2} a_{r s}\left(q_{1}, \ldots, q_{d / 2}\right) \bar{p}_{s} .
\end{aligned}
$$


It follows that $\bar{\varphi}_{O}^{\mathscr{B}} \circ \tau_{O}^{\mathscr{B}}-1$ is bijective and that $\tau_{O}^{\mathscr{B}} \circ \bar{\varphi}_{O}^{\mathscr{B}}-1$ is polynomial. This ends the proof of the lemma.

Before going on, let us note that we have

$$
\{\varphi, \psi\}=\sum_{r, s=1}^{d / 2}\left\{\bar{p}_{r}, \bar{p}_{s}\right\} \frac{\partial \varphi}{\partial \bar{p}_{r}} \frac{\partial \psi}{\partial \bar{p}_{s}}+\sum_{r=1}^{d / 2}\left(\frac{\partial \varphi}{\partial \bar{p}_{r}} \frac{\partial \psi}{\partial q_{r}}-\frac{\partial \varphi}{\partial q_{r}} \frac{\partial \psi}{\partial \bar{p}_{r}}\right) .
$$

In particular we have

$$
\left\{\bar{p}_{r}, \psi\right\}=\sum_{s=1}^{d / 2}\left\{\bar{p}_{r}, \bar{p}_{s}\right\} \frac{\partial \psi}{\partial \bar{p}_{s}}+\frac{\partial \psi}{\partial q_{r}} .
$$

If $\psi \in \mathscr{P}_{F(O, \mathscr{F})}^{0}(O)$ it follows that

$$
\left\{\bar{p}_{r}, \psi\right\}=\frac{\partial \psi}{\partial q_{r}} .
$$

Lemma 5.4.5. Let $q_{1}, \ldots, q_{d / 2}$ be generators of $\mathscr{P}_{F(O, \mathscr{F})}^{0}(O)$, and let $\bar{p}_{1}, \ldots$, $\bar{p}_{d / 2}$ be elements in $\mathscr{P}_{F(O, \mathscr{F})}^{1}(O)$ such that $\left\{\bar{p}_{u}, q_{v}\right\}=\delta_{u v}, \quad 1 \leq u, v \leq d / 2$. Then there exists functions $a_{1}, \ldots, a_{d / 2} \in \mathscr{P}_{F(O, \mathscr{F})}^{0}(O)$ such that if we set $p_{u}=$ $\bar{p}_{u}+a_{u}, 1 \leq u \leq d / 2$, then $\left\{p_{u}, q_{v}\right\}=\delta_{u v}$ and $\left\{p_{u}, p_{v}\right\}=0$ for $1 \leq u, v \leq$ $d / 2$.

Proof. We shall give a recursive way of obtaining such functions $a_{u}$ : We set $a_{d / 2}=0$. Suppose that $a_{d / 2}, \ldots, a_{j}$ have been determined such that $\left\{p_{r}, p_{s}\right\}=$ 0 for $j \leq r, s \leq d / 2$ and $\left\{p_{r}, q_{s}\right\}=\delta_{r s}, 1 \leq s \leq d / 2, j \leq r \leq d / 2$. We seek an element $a_{j-1} \in \mathscr{P}_{F(O, \mathscr{F})}^{0}(O)$ such that $p_{j-1}=\bar{p}_{j-1}+a_{j-1}$ satisfies $\left\{p_{j-1}, q_{s}\right\}=\delta_{j-1 s}$ for $1 \leq s \leq d / 2$ and $\left\{p_{j-1}, p_{s}\right\}=0$ for $j \leq s \leq d / 2$, i.e., such that $\left\{\bar{p}_{j-1}, q_{s}\right\}=\delta_{j-1 s}$ and $\left\{\bar{p}_{j-1}, p_{s}\right\}=-\left\{a_{j-1}, p_{s}\right\}$ for $j \leq s \leq d / 2$. But the first condition is already satisfied, so the element $a_{j-1}$ has to satisfy $\left\{\bar{p}_{j-1}, p_{s}\right\}=-\left\{a_{j-1}, p_{s}\right\}=-\left\{a_{j-1}, \bar{p}_{s}\right\}$ for $j \leq s \leq d / 2$. Now since $a_{j-1}$ is assumed to be in $\mathscr{P}_{F(O, \mathscr{F})}^{0}(O)$ we must have $\left\{a_{j-1}, \bar{p}_{s}\right\}=-\partial a_{j-1} / \partial q_{s}$ which means that it is necessary and sufficient to find $a_{j-1}$ in $\mathscr{P}_{F(O, \mathscr{F})}^{0}(O)$ such that

$$
\frac{\partial a_{j-1}}{\partial q_{s}}=\left\{\bar{p}_{j-1}, p_{s}\right\}
$$

for $s=d / 2, \ldots, j$. We shall show that this system of equations can be solved. Set $f_{s}=\left\{\bar{p}_{j-1}, p_{s}\right\}$ for $s=d / 2, \ldots, j$. First we claim that $f_{s} \in$ $\mathscr{P}_{F(O, F)}^{0}(O), j \leq s \leq d / 2$. To see this, note that $\left\{q_{s}, f_{r}\right\}=-\left\{q_{s},\left\{p_{r}, p_{j-1}\right\}\right\}=$ $\left\{p_{r},\left\{\bar{p}_{j-1}\right\}\right\}+\left\{\bar{p}_{j-1},\left\{q_{s}, p_{r}\right\}\right\}=0$, since either $\left\{q_{s}, p_{r}\right\}=0$ or $=1$ and similarly for $\left\{\bar{p}_{j-1}, q_{s}\right\}$. This means that we can consider $f_{1}, \ldots, f_{d / 2}$ as functions of $q_{1}, \ldots, q_{d / 2}$, and that we have to solve the equations

$$
\frac{\partial a_{j-1}}{\partial q_{s}}=f_{s}\left(q_{1}, \ldots, q_{d / 2}\right), \quad r=d / 2, \ldots, j .
$$


Since we are in a simply connected domain these equations can be solved if and only if we have

i.e., if and only if

$$
\frac{\partial f_{r}}{\partial q_{s}}=\frac{\partial f_{s}}{\partial q_{r}} \quad \text { for } j \leq r, s \leq d / 2,
$$

$$
\left\{p_{s}, f_{r}\right\}=\left\{p_{r}, f_{s}\right\} \text { for } j \leq r, s \leq d / 2 \text {. }
$$

But for $j \leq r, s \leq d / 2$ we have

$$
\begin{aligned}
\frac{\partial f_{r}}{\partial q_{s}} & =\left\{p_{s}, f_{r}\right\}=\left\{p_{s},\left\{p_{r}, \bar{p}_{j-1}\right\}\right\} \\
& =-\left\{p_{r},\left\{\bar{p}_{j-1}, p_{s}\right\}\right\}-\left\{\bar{p}_{j-1},\left\{p_{s}, p_{r}\right\}\right\} \\
& =\left\{p_{r},\left\{p_{s}, \bar{p}_{j-1}\right\}\right\}=\left\{p_{r},\left\{p_{s}, \bar{p}_{j-1}\right\}\right\}=\frac{\partial f_{s}}{\partial q_{r}}
\end{aligned}
$$

and this shows that these equations can be solved. Since the functions $f_{r}$ are polynomials in $\mathbf{C}\left[q_{1}, \ldots, q_{d / 2}\right]$, there is an obvious algorithm for for finding $a_{j-1}$. Note also that the functions $a_{u}$ are uniquely determined if we require that $p_{u}(0, \ldots, 0)=0$. This end the proof of the lemma.

We now use Lemma 5.4 .5 on the functions $\bar{p}_{1}, \ldots, \bar{p}_{d / 2}$ constructed previously to find functions $p_{1}=p_{1}^{O}, \ldots, p_{d / 2}=p_{d / 2}^{O}$ satisfying the properties in Lemma 5.4.5. We define $\varphi_{O}: O \rightarrow \mathbf{R}^{d}$ by

$$
\varphi_{O}: l \rightarrow\left(p_{1}^{o}(l), \ldots, p_{d / 2}^{o}, q_{1}^{o}(l), \ldots, q_{d / 2}^{o}(l)\right) .
$$

It is immediate that $\varphi_{O} \circ \bar{p}_{O}^{-1}$ is a bi-polynomial diffeomorphism. We have thus constructed a polynomial global chart on $O$.

5.4.6. Using the polynomial global chart $\varphi_{O}=\left(p_{1}, \ldots, p_{d / 2}, q_{1}, \ldots, q_{d / 2}\right)$ we have that $\mathscr{P}_{F}^{0}(O)$ consists of functions of the form $a(q)$, where $a \in \mathscr{P}\left(\mathbf{R}^{d / 2}\right)$. It follows that each function $\varphi \in \mathscr{P}_{F}^{1}(O)$ can be written uniquely as

$$
\varphi=\sum_{r=1}^{d / 2} b_{r}(q) \psi^{X_{k_{r}}}+b_{0}(q),
$$

where $b_{0}, b_{1}, \ldots, b_{d / 2}$ belong to $\mathscr{P}\left(\mathbf{R}^{d / 2}\right)$ (Proposition 2.2.9). In particular each $\psi^{X}, X \in \mathfrak{g}$, can be written

$$
\psi^{X}=\sum_{r=1}^{d / 2} b_{X, r}(q) \psi^{X_{k_{r}}}+b_{X, 0}(q) .
$$

Setting $b_{s, r}=b_{X_{j_{s}}, r}$ and using the notation from the beginning of $\S 5.4$ this means that we can write each $y_{j_{s}}$ uniquely as

$$
y_{j_{s}}=\sum_{r=1}^{d / 2} b_{s, r}(q) y_{k_{r}}+b_{s, 0}(q) \text {, }
$$


$s=1, \ldots, d$. Suppose now that $j_{s} \in L_{g}$. Then there exists $1 \leq w \leq d / 2$ such that $j_{s}=\bar{l}_{w}$, and we have

$$
\begin{aligned}
y_{j_{s}} & =h_{g, j_{s}}(z)=z_{j_{s}}+\bar{h}_{j_{s}}\left(g, z_{j_{1}}, \ldots, z_{j_{s-1}}\right) \\
& =q_{w}+\bar{h}_{j_{s}}\left(g, z_{j_{1}}, \ldots, z_{j_{s-1}}\right),
\end{aligned}
$$

where we use the notation from the proof of Lemma 5.4.1. Comparing this with the expression above we get that

$$
y_{j_{s}}=y_{\bar{l}_{w}}=q_{w}+\sum_{r=1}^{d / 2} b_{s, r}\left(q_{1}, \ldots, q_{w-1}\right) y_{k_{r}}+b_{0, r}^{\prime}\left(q_{1}, \ldots, q_{w-1}\right),
$$

where $b_{s, 0}^{\prime}(q)=-q_{w}+b_{s, 0}\left(q_{1}, \ldots, q_{w-1}\right)$.

Let us then use the polynomial canonical coordinates $\varphi_{O}$ to construct the representation $\pi$ as in Theorem 2.7.2. It is then immediate that we can construct elements $v_{w} \in U\left(\mathfrak{g}_{\mathbf{C}}\right), w=1, \ldots, d / 2$, such that $d \pi\left(v_{w}\right)=i M_{w}$, where $M_{w} f(t)=t_{w} f(t)$. In fact, set $v_{1}=X_{j_{1}}\left(=X_{\bar{l}_{1}}\right)$; then $d \pi\left(v_{1}\right)=i M_{1}$. Suppose then that we have found $v_{1}, \ldots, v_{w-1}, w<d / 2$, such that $d \pi\left(v_{r}\right)=i M_{r}$ for $1 \leq r \leq w-1$, and let $1 \leq s \leq d$ be such that $\bar{l}_{w}=j_{s}$. It then follows from Theorem 2.7.2 that

$$
\begin{aligned}
d \pi\left(X_{j_{s}}\right)= & i M_{w}+\sum_{r=1}^{d / 2} b_{r, s}\left(M_{1}, \ldots, M_{w-1}\right) d \pi\left(X_{k_{r}}\right)+i b_{0, r}^{\prime}\left(M_{1}, \ldots, M_{w-1}\right) \\
= & i M_{w}+\sum_{r=1}^{d / 2} b_{r, s}\left(-i d \pi\left(v_{1}\right), \ldots,-i d \pi\left(v_{w-1}\right)\right) d \pi\left(X_{k_{r}}\right) \\
& +i b_{0, r}^{\prime}\left(-i d \pi\left(v_{1}\right), \ldots,-i d \pi\left(v_{w-1}\right)\right) \\
= & i M_{w}+d \pi\left(\sum_{r=1}^{d / 2} b_{r, s}\left(-i v_{1}, \ldots,-i v_{w-1}\right) X_{k_{r}}\right) \\
& +i d \pi\left(b_{0, r}^{\prime}\left(-i v_{1}, \ldots,-i v_{w-1}\right)\right) .
\end{aligned}
$$

Therefore, if we set

$$
v_{u}=X_{j_{s}}-\sum_{r=1}^{d / 2} b_{r, s}\left(-i v_{1}, \ldots,-i v_{w-1}\right) X_{k_{r}}-i b_{0, r}^{\prime}\left(-i v_{1}, \ldots,-i v_{w-1}\right),
$$

we have that $d \pi\left(v_{w}\right)=i M_{w}$. It is then clear that we can also find elements $u_{r} \in U\left(\mathfrak{g}_{\mathbf{C}}\right)$ such that $d \pi\left(u_{r}\right)=D_{r}$ (Proposition 2.3.1), and it follows that $d \pi\left(U\left(\mathfrak{g}_{\mathrm{C}}\right)\right)=\mathscr{A}^{d / 2}\left(\mathbf{R}^{d / 2}\right)$. This result is of course immediate from Theorem 3.2.1. The point is, however, that here we have proved this result without the usual induction-on-the-dimension-of-the-group argument. Having this result it follows easily that the representation $\pi$ is irreducible.

This means that we have now, for the first time, a noninductive proof of the fact that one can associate a well-defined class of irreducible representations 
of the group $G$ with each coadjoint orbit. In fact, here we have proved that the representation constructed by means of a Vergne polarization is irreducible, and it follows by a noninductive argument from [10] that any representations arising from a polarization satisfies the Kirillov character formula. Since we have proved here that at least one of these representations is irreducible, they all are, and, moreover, they are all equivalent.

We note that we have also given, for the first time, a noninductive proof of the fact that the image by the differential of an irreducible representation associated with an orbit is isomorphic to the Weyl algebra $\mathscr{A}\left(\mathbf{R}^{d / 2}\right)$.

Let us finally remark that there are other basic results from the Kirillov theory that still lack noninductive proofs; the most important of these are the fact that all irreducible representaions are associated with orbits, and the fact that the center of the universal enveloping algebra is isomorphic to the algebra of all $G$-invariant polynomials on $\mathfrak{g}^{*}$ (where we even have an explicit isomorphism using the symmetrization map).

5.5. The rational functions $p^{\varepsilon}$. We shall now define the rational functions $p_{r}^{\varepsilon}$ appearing in Theorem 5.1.1.

First we consider the functions $\bar{p}_{r}^{O}$ defined in $\S 5.4$. We start by noting that if $\varphi, \psi \in \mathscr{E}(O)$, then

$$
\{\varphi, \psi\}=\sum_{u, v=1}^{d}\left(\sum_{k=1}^{m} c_{j_{u} j_{v}} R_{k}(g, y)\right) \frac{\partial \varphi}{\partial y_{j_{u}}} \frac{\partial \psi}{\partial y_{j_{v}}},
$$

see $\S 2.1$. It follows that if we let $\alpha_{r s}(g, y)$ denote the entries of the matrix $\mathbf{A}_{12}\left(\sigma_{O}^{\mathscr{B}}-1(y)\right), O=G g$, where $\mathbf{A}_{12}(l)$ is defined in $\S 5.4$, we get with, the notation from the proof of Lemma 5.4.1, that

$$
\alpha_{r s}(g, y)=\sum_{u, v=1}^{d}\left(\sum_{k=1}^{m} c_{j_{u} j_{v} k} R_{k}(g, y)\right) \frac{\partial \mathfrak{f}_{k_{r}}}{\partial y_{j_{u}}}(g, y) \frac{\partial f_{\bar{l}_{s}}}{\partial y_{j_{v}}}(g, y),
$$

and from this it follows that we can extend $\alpha_{r s}(g, y)$ in a natural way to a rational function on $\mathfrak{g}^{*}$ with denominator in the algebra generated by the polynomials $P_{e_{1}}, \ldots, P_{e_{m}}$. Let then $\beta_{r s}(g, y)$ denote the entries of the matrix $\mathbf{B}\left(\sigma_{O}^{\mathscr{B}}-1(y)\right)$, where also the matrix $\mathbf{B}(l)=\mathbf{A}_{12}(l)^{-1}$ is defined in $\S 5.4$. Since $\operatorname{det} \mathbf{B}(l)=P_{e_{m}}(l)$ for $l \in \Omega_{\varepsilon}$ we see that we can extend $g \rightarrow \beta_{r s}(g, y)$ to a rational function on $\mathfrak{g}^{*}$ with denominators in the algebra generated by the polynomials $P_{e_{1}}, \ldots, P_{e_{m}}$. We then set

$$
\bar{F}_{r}(g, y)=\sum_{s=1}^{d / 2} \beta_{r s}(g, y) f_{k_{s}}(g, y), \quad r=1, \ldots, d / 2 .
$$

The functions $(g, y) \rightarrow \bar{F}_{r}(g, y)$ are the polynomials in the variable $y$ and rational functions the variable $g$ with denominators in the algebra generated 
by the polynomials $P_{e_{1}}, \ldots, P_{e_{m}}$, and it follows from $\S 5.4$ that if $g \in \Omega_{\varepsilon}$ and $O=G g$, then

$$
\bar{p}_{r}^{O}(y)=\bar{F}_{r}(g, y) \text {. }
$$

Having this result we proceed to consider the functions $p_{r}^{O}$ constructed using Lemma 5.4.5. In the proof of Lemma 5.4.5 we constructed $p_{d}^{O}, \ldots, p_{1}^{O}$ be a recursion procedure. Inspecting this procedure, using the result we have already obtained about $\bar{p}_{r}^{O}$, and arguing as above we see that there exists functions $F_{r}(g, y)$ that are polynomial in the variable $y$ and a rational function in the variable $g$ with denominators in the algebra generated by $P_{e_{1}}, \ldots, P_{e_{m}}$, and such that

$$
p_{r}^{O}\left(\sigma_{O}^{\mathscr{B}-1}(y)\right)=F_{r}(g, y),
$$

when $g \in \Omega_{\varepsilon}, O=G g, r=1, \ldots, d / 2$.

We now define the functions $p_{r}^{\varepsilon}$ by

$$
p_{r}^{\varepsilon}(g)=F_{r}\left(R_{1}(g, 0), \ldots, R_{m}(g, 0), \xi_{j_{1}}, \ldots, \xi_{j_{d}}\right),
$$

where we identify elements $g \in \mathfrak{g}^{*}$ with their coordinates $\xi_{j}=\left\langle g, X_{j}\right\rangle$ with respect to the basis in $\mathfrak{g}^{*}$ dual to $X_{1}, \ldots, X_{m}$. Clearly the functions $p_{r}^{\varepsilon}, r=$ $1, \ldots, d / 2$, are rational functions with denominators in the algebra generated by $P_{e_{1}}, \ldots, P_{e_{m}}$, and we have

$$
p_{r}^{O}=p_{r}^{\varepsilon} \mid O, \quad r=1, \ldots, d / 2 ;
$$

in fact, let $l \in O$, and set $y=\sigma_{O}^{\mathscr{B}}(l)$. Then $\left\langle l, X_{j_{r}}\right\rangle=y_{j_{r}}$, so

$$
\begin{aligned}
p_{r}^{O}(l) & =F_{r}(l, y) \\
& =F_{r}\left(R_{1}(l, 0), \ldots, R_{m}(l, 0), y_{j_{1}}, \ldots, y_{j_{d}}\right) \\
& =p_{r}^{\varepsilon}(l) .
\end{aligned}
$$

We have now found the rational functions $p_{r}^{\varepsilon}$ and $q_{r}^{\varepsilon}$ from Theorem 5.1.1, and this ends the proof of Theorem 5.1.1. Let $\mathfrak{g}$ be the 6-dimensional nilpotent Lie algebra with a basis $\mathscr{B}: X_{1}, \ldots, X_{6}$ satisfying the following nonvanishing commutation relations (we only give $\left[X_{i}, X_{j}\right]$ for $i>j$ ):

$$
\begin{aligned}
& {\left[X_{6}, X_{5}\right]=X_{4},\left[X_{6}, X_{4}\right]=X_{3},\left[X_{6}, X_{3}\right]=X_{2},} \\
& {\left[X_{5}, X_{4}\right]=X_{2},\left[X_{5}, X_{2}\right]=-X_{1},\left[X_{4}, X_{3}\right]=X_{1} .}
\end{aligned}
$$

We only carry out the calculations in the first stratum. The other strata are handled similarly. The canonical coordinates $\left(p_{1}, p_{2}, q_{1}, q_{2}\right)$ have been calculated using the algorithm from $\S 5$.

1 st stratum: $\xi_{1} \neq 0$ :

The 5-tuple of jump indices: $\varepsilon=(\varnothing, \varnothing, \varnothing,\{3,4\},\{2,3,4,5\},\{2,3,4,5\})$

The dimension of orbits: $d=4$. 
The jump indices:

$j_{1}=2, j_{2}=3, j_{3}=4, j_{4}=5$,

$k_{1}=4, k_{2}=5$,

$l_{1}=3, l_{2}=2$,

$\bar{l}_{1}=2, \bar{l}_{2}=3$.

The polynomials $P_{e_{j}}$ :

$P_{e_{3}}=\xi_{1}$,

$P_{e_{4}}=-\xi_{1}^{2}$.

The functions $\psi^{X_{j}}=R_{j}$ expressed in the coordinates $\left(y_{2}, y_{3}, y_{4}, y_{5}\right)$ :

$R_{1}(g, y)=y_{1}=\xi_{1}$,

$R_{2}(g, y)=y_{2}$,

$R_{3}(g, y)=y_{3}$,

$R_{4}(g, y)=y_{4}$,

$R_{5}(g, y)=y_{5}$,

$R_{6}(g, y)=y_{6}=\xi_{6}+\frac{1}{6 \xi(1)^{2}}\left(-6 \xi_{1} \xi_{2} \xi_{4}+3 \xi_{1} \xi_{3}^{2}-2 \xi_{2}^{3}+6 \xi_{1} y_{2} y_{4}-3 \xi_{1} y_{3}^{2}+2 y_{2}^{3}\right)$.

The rational functions $\left(p_{1}^{\varepsilon}, p_{2}^{\varepsilon}, q_{1}^{\varepsilon}, q_{2}^{\varepsilon}\right)$ :

$p_{1}^{\varepsilon}=\frac{1}{\xi_{1}^{2}}\left(-\xi_{1} \xi_{5}-\xi_{2} \xi_{3}\right)$,

$p_{2}^{e}=\frac{1}{\xi_{1}} \xi_{4}$

$q_{1}^{\varepsilon}=\xi_{2}$,

$q_{2}^{\varepsilon}=\xi_{3}$.

The canonical coordinates $\left(p_{1}, p_{2}, q_{1}, q_{2}\right)$ expressed in the coordinates $\left(y_{2}, y_{3}\right.$, $\left.y_{4}, y_{5}\right)$ :

$$
\begin{aligned}
p_{1} & =\frac{1}{\xi_{1}^{2}}\left(-\xi_{1} y_{5}-y_{3} y_{2}\right), \\
p_{2} & =\frac{1}{\xi_{1}} y_{4}, \\
q_{1} & =y_{2}, \\
q_{2} & =y_{3} .
\end{aligned}
$$

The functions $\psi^{X_{j}}$ expressed in the canonical coordinates $\left(p_{1}, p_{2}, q_{1}, q_{2}\right)$ :

$$
\begin{aligned}
& y_{1}=\xi_{1}, \\
& y_{2}=q_{1},
\end{aligned}
$$




$$
\begin{aligned}
& y_{3}=q_{2}, \\
& y_{4}=\xi_{1} p_{2}, \\
& y_{5}=\frac{1}{\xi_{1}}\left(-\xi_{1}^{2} p_{1}-q_{2} q_{1}\right), \\
& y_{6}=\frac{1}{6 \xi_{1}^{2}}\left(6 \xi_{1}^{2} \xi_{6}+6 \xi_{1}^{2} q_{1} p_{2}-6 \xi_{1} \xi_{2} \xi_{4}+3 \xi_{1} \xi_{3}^{2}-3 \xi_{1} q_{2}^{2}-2 \xi_{2}^{3}+2 q_{1}^{3}\right) .
\end{aligned}
$$

The representations calculated from the formula in Theorem 3.2.1 using the global canonical coordinates $\left(p_{1}, p_{2}, q_{1}, q_{2}\right)$ :

$$
\begin{aligned}
& d \pi\left(X_{1}\right)=i \xi_{1}, \\
& d \pi\left(X_{2}\right)=i t_{1}, \\
& d \pi\left(X_{3}\right)=i t_{2}, \\
& d \pi\left(X_{4}\right)=\xi_{1} \frac{\partial}{\partial t_{2}} \\
& d \pi\left(X_{5}\right)=\frac{1}{\xi_{1}}\left(-\xi_{1}^{2} \frac{\partial}{\partial t_{1}}-i t_{1} t_{2}\right), \\
& d \pi\left(X_{6}\right)=\frac{1}{6 \xi_{1}^{2}}\left(6 \xi_{1}^{2} \xi_{6}+6 \xi_{1}^{2} t_{1} \frac{\partial}{\partial t_{2}}-6 \xi_{1} \xi_{2} \xi_{4}+3 \xi_{1} \xi_{3}^{2}-3 i \xi_{1} t_{2}^{2}-2 \xi_{2}^{3}+2 i t_{1}^{3}\right) .
\end{aligned}
$$

The elements $u_{1}, u_{2}, v_{1}, v_{2} \in U\left(\mathfrak{g}_{\mathrm{C}}\right)$ defined in $\S 3.2$, cf. Theorem 3.2.1:

$$
\begin{aligned}
& u_{1}=\frac{1}{\xi_{1}^{2}}\left(\xi_{1} X_{5}+i X_{3} X_{2}\right), \\
& u_{2}=\frac{1}{\xi_{1}} X_{4}, \\
& v_{1}=X_{2}, \\
& v_{2}=X_{3} .
\end{aligned}
$$

Added in proof. In a recent paper by $\mathrm{P}$. Bonnet, Paramétrisation du dual d'une algèbre de Lie nilpotente, Ann. Inst. Fourier (Grenoble) 38 (1988), 169-197, results similar to those of $\S 5$ in the present paper have been obtained.

\section{REFERENCES}

1. D. Arnal, J. C. Cortet, P. Molin and G. Pinczon, Covariance and geometrical invariance in *-quantization, J. Math. Phys. 24 (1983), 276-283.

2. L. Corwin, F. P. Greenleaf and R. Penney, A general character formula for irreducible projections in $L^{2}$ of a nilmanifold, Math. Ann. 225 (1977), 21-32.

3. J. Dixmier, Algèbres enveloppantes, Gauthier-Villars, Paris, 1974.

4. J. Dixmier, Représentations irréductibles des algèbres de Lie nilpotents, An. Acad. Brasil Ciênc. 53 (1963), 491-519.

5. A. A. Kirillov, Unitary representations of nilpotent Lie groups, Uspehi Mat. Nauk 17 (1962), 57-110.

6. A. I. Malcev, On a class of homogeneous spaces, Amer. Math. Soc. Transl. (1) 39 (1951).

7. N. V. Pedersen, On the infinitesimal kernel of irreducible representations of nilpotent Lie groups, Bull. Soc. Math. France 112 (1984), 423-467. 
8. __ Characters of solvable Lie groups, Harmonische Analyse und Darstellungstheorie topologischer Gruppen, Tagungsbericht 33/1985, Mathematisches Forschungsinstitut, Oberwolfach, 1985.

9. $\ldots$, On the symplectic structure of coadjoint orbits of (solvable) Lie groups and applications. I, Math. Ann. 281 (1988), 633-669.

10. L. Pukanszky, On the characters and the Plancherel formula of nilpotent groups, J. Funct. Anal. 1 (1967), 255-280.

11. M. Vergne, La structure de Poisson sur l'algèbre symmétrique d'une algèbre de Lie nilpotente, Bull. Soc. Math. France 100 (1972), 301-335.

Mathematical Institute, University of Copenhagen, Universitetsparken 5, DK-2100 Copenhagen, Denmark 